August 27, $2020 \quad$ 1:16 WSPC/INSTRUCTION FILE BBsubschemes-final

\title{
COMPUTING SUBSCHEMES OF THE BORDER BASIS SCHEME
}

\author{
MARTIN KREUZER \\ Fakultät für Informatik und Mathematik, Universität Passau, D-94030 Passau, Germany \\ Martin.Kreuzer@uni-passau.de \\ LE NGOC LONG \\ Fakultät für Informatik und Mathematik, Universität Passau, D-94030 Passau, Germany and \\ Department of Mathematics, University of Education - Hue University, 34 Le Loi, Hue, \\ Vietnam \\ lelong@hueuni.edu.vn \\ LORENZO ROBBIANO \\ Dipartimento di Matematica, Università di Genova, Via Dodecaneso 35, I-16146 Genova, Italy \\ lorobbiano@gmail.com
}

\begin{abstract}
A good way of parametrizing 0-dimensional schemes in an affine space $\mathbb{A}_{K}^{n}$ has been developed in the last 20 years using border basis schemes. Given a multiplicity $\mu$, they provide an open covering of the Hilbert scheme $\operatorname{Hilb}^{\mu}\left(\mathbb{A}_{K}^{n}\right)$ and can be described by easily computable quadratic equations. A natural question arises on how to determine loci which are contained in border basis schemes and whose rational points represent 0 -dimensional $K$-algebras sharing a given property. The main focus of this paper is on giving effective answers to this general problem. The properties considered here are the locally Gorenstein, strict Gorenstein, strict complete intersection, Cayley-Bacharach, and strict Cayley-Bacharach properties. The key characteristic of our approach is that we describe these loci by exhibiting explicit algorithms to compute their defining ideals. All results are illustrated by non-trivial, concrete examples.
\end{abstract}

Keywords: zero-dimensional ideal, border basis, border basis scheme, Cayley-Bacharach property, Gorenstein ring, strict complete intersection

Mathematics Subject Classification 2010: Primary 13C40, Secondary 14M10, 13H10, 13P99, 14Q99

\section{Introduction}

In [9], A. Grothendieck defined the Hilbert scheme $\operatorname{Hilb}^{\mu}\left(\mathbb{A}_{K}^{n}\right)$ which parametrizes all 0-dimensional subschemes of length $\mu$ of $\mathbb{A}_{K}^{n}$ as a quotient of a suitable Grassmannian variety. If we turn this construction into a computable presentation, its defining relations yield a rather large and unwieldy system of equations (see, for instance, [13]). A more convenient way of parametrizing 0-dimensional schemes in $\mathbb{A}_{K}^{n}$, or equivalently, of 0 -dimensional affine algebras over a field $K$, has been developed in the last 20 years, namely the border basis scheme (see [10], 11], 20], 21], and [22]). 
The border basis schemes corresponding to order ideals of length $\mu$ form an open covering of the Hilbert scheme $\operatorname{Hilb}^{\mu}\left(\mathbb{A}_{K}^{n}\right)$, and they have simple, explicit presentations involving easily describable quadratic equations. Having such explicit descriptions of large families of 0-dimensional schemes, it is a natural question to ask for similar descriptions of the subsets given by all 0-dimensional schemes having certain geometric or algebraic properties. This is the task that we tackle here, and we treat algebraic properties such as the locally Gorenstein, strict Gorenstein and strict complete intersection properties, as well as geometric properties such as the Cayley-Bacharach and strict Cayley-Bacharach properties. Notice that, for instance, although the locally Gorenstein locus of the Hilbert scheme is actively researched (e.g., see [2], 3, 4], 5]), the simple explicit defining equations computed here appear to be new.

In more detail, we proceed as follows. In Sections 2 and 3, we recall some basic material about border bases and border basis schemes, respectively. Let $\mathcal{O}=\left\{t_{1}, \ldots, t_{\mu}\right\}$ be an order ideal of terms in $K\left[x_{1}, \ldots, x_{n}\right]$, i.e., a factorclosed finite set of power products of indeterminates. We define its border $\partial \mathcal{O}=$ $\left(\bigcup_{i=1}^{n} x_{i} \mathcal{O}\right) \backslash \mathcal{O}=\left\{b_{1}, \ldots, b_{\nu}\right\}$ and the generic $\mathcal{O}$-border prebasis $G=\left\{g_{1}, \ldots, g_{\nu}\right\}$, where $g_{j}=b_{j}-\sum_{i=1}^{\mu} c_{i j} t_{i}$ and where $C=\left\{c_{i j}\right\}$ is a set of new indeterminates. Then the border basis scheme $\mathbb{B}_{\mathcal{O}}$ is the subscheme of $\mathbb{A}_{K}^{\mu \nu} \cong \operatorname{Spec}(K[C])$ defined by the vanishing of the entries of the commutators of the generic multiplication matrices for $K[C]\left[x_{1}, \ldots, x_{n}\right] /\langle G\rangle$. Notice that the scheme $\mathbb{B}_{\mathcal{O}}$ may be non-reduced and can have irreducible components of higher dimension than the expected dimension $n \mu$ (see 20, Example 5.6). Nevertheless it is defined by a set of nice and easy quadratic polynomials in the indeterminates $c_{i j}$.

In Section 4 we start by looking at the subscheme of $\mathbb{B}_{\mathcal{O}}$ defined by the property that the affine coordinate ring $R_{\mathbb{X}}$ of the scheme $\mathbb{X}$ represented by a $K$-rational point of $\mathbb{B}_{\mathcal{O}}$ is locally Gorenstein. This ring-theoretic property has been characterized in [14 by the condition that a certain determinant of a matrix constructed from the multiplication matrices of $R_{\mathbb{X}}$ with respect to the basis $\mathcal{O}$ is non-zero. The global version of this characterization yields Algorithm 4.3, where the equations defining the locally Gorenstein locus in $\mathbb{B}_{\mathcal{O}}$ are calculated explicitly.

Other loci require us to stratify the border basis scheme before we can compute their defining ideals. A first important step in this direction is taken in Section 5, where we define and study the degree filtered border basis scheme $\mathbb{B}_{\mathcal{O}}^{\mathrm{df}}$. This is the subscheme of $\mathbb{B}_{\mathcal{O}}$ whose $K$-rational points represent schemes $\mathbb{X}$ such that $\mathcal{O}$ is a degree filtered $K$-basis of $R_{\mathbb{X}}$, i.e., such that the elements of degree $\leq i$ in $\mathcal{O}$ form a $K$-basis of the $i$-th part of the degree filtration of $R_{\mathbb{X}}$ for every $i \geq 0$. The scheme $\mathbb{B}_{\mathcal{O}}^{\mathrm{df}}$ is the basic Hilbert stratum of $\mathbb{B}_{\mathcal{O}}$, and the computation of various loci in other Hilbert strata will be reduced to this case later in the paper.

Sections 6,7 , and 8 provide several algorithms which are central to this paper. Firstly, we compute the Cayley-Bacharach locus in $\mathbb{B}_{\mathcal{O}}^{\text {df }}$ in Algorithm 6.3 Here we use the results of 14] again and generalize them to the universal border basis family. Recall that the Cayley-Bacharach property of a 0 -dimensional scheme is a geometric 
property which has been generalized multiple times in the history of Mathematics (see the introduction of [14]) and is used here in its most general form: over an arbitrary base field $K$, and for an arbitrary 0 -dimensional scheme $\mathbb{X}$ in $\mathbb{A}_{K}^{n}$.

Secondly, we compute the strict Cayley-Bacharach locus in $\mathbb{B}_{\mathcal{O}}^{\text {df }}$ in Algorithm 7.10. The adjective "strict" indicates that we are looking for the locus in $\mathbb{B}_{\mathcal{O}}^{\mathrm{df}}$ whose $K$-rational points represent schemes $\mathbb{X}$ such that the graded $\operatorname{ring} \operatorname{gr}_{\mathcal{F}}\left(R_{\mathbb{X}}\right)$ of $R_{\mathbb{X}}$ with respect to the degree filtration has the Cayley-Bacharach property. Geometrically, this means that also the tip of the affine cone over $\mathbb{X}$ has this property, and in projective geometry, the corresponding adjective is "arithmetical". Algorithm 7.10 is based on the homogeneous border basis scheme $\mathbb{B}_{\mathcal{O}}^{\text {hom }}$, recalled in Section 5, which parametrizes the rings $\operatorname{gr}_{\mathcal{F}}\left(R_{\mathbb{X}}\right)$, and also on Algorithm 7.2 which allows us to check the strict Cayley-Bacharach property for a single scheme $\mathbb{X}$ using its multiplication matrices.

Thirdly, the strict complete intersection locus in $\mathbb{B}_{\mathcal{O}}^{\text {df }}$ is computed in Algorithm 8.3. Again, the adjective "strict" refers to the $\operatorname{ring} \operatorname{gr}_{\mathcal{F}}\left(R_{\mathbb{X}}\right)$ being a complete intersection, or, equivalently, that $R_{\mathbb{X}}=K\left[x_{1}, \ldots, x_{n}\right] /\left\langle f_{1}, \ldots, f_{n}\right\rangle$ where the degree forms $\left\{\mathrm{DF}\left(f_{1}\right), \ldots, \mathrm{DF}\left(f_{n}\right\}\right\}$ form a (homogeneous) regular sequence. For one scheme $\mathbb{X}$, an algorithm for checking this property using the multiplication matrices of $R_{\mathbb{X}}$ was developed in [15. Here we extend this method to the universal border basis family and get Algorithm 7.10

The further parts of the paper focus on using these results to describe the corresponding loci in all of $\mathbb{B}_{\mathcal{O}}$. To this end, we introduce and compute the Hilbert stratification of $\mathbb{B}_{\mathcal{O}}$ in Section 9. Given an admissible Hilbert function $\mathcal{H}$, we first compute the closed subscheme $\mathbb{B}_{\mathcal{O}}(\overline{\mathcal{H}})$ of $\mathbb{B}_{\mathcal{O}}$ whose $K$-rational points represent schemes such that their affine Hilbert function is dominated by $\mathcal{H}$, and then the open subscheme $\mathbb{B}_{\mathcal{O}}(\mathcal{H})$ of $\mathbb{B}_{\mathcal{O}}(\overline{\mathcal{H}})$ which is the locus where the affine Hilbert function is exactly $\mathcal{H}$.

However, for several applications, we even have to fix a degree filtered basis of the ring $R_{\mathbb{X}}$. This is achieved in Section 10 by covering $\mathbb{B}_{\mathcal{O}}(\mathcal{H})$ with open subschemes $\mathbb{B}_{\mathcal{O}}^{\mathrm{dfb}}\left(\mathcal{O}^{\prime}\right)$, called $\mathcal{O}^{\prime}$-DFB subschemes, such that the order ideal $\mathcal{O}^{\prime}$ is a degree filtered $K$-basis of $R_{\mathbb{X}}$ for all schemes $\mathbb{X}$ represented by $K$-rational points of $\mathbb{B}_{\mathcal{O}}^{\mathrm{dfb}}\left(\mathcal{O}^{\prime}\right)$. The ideals describing the various schemes $\mathbb{B}_{\mathcal{O}}^{\mathrm{dfb}}\left(\mathcal{O}^{\prime}\right)$ are computed in Algorithm 10.2. Then the key result for the last parts of the paper is Algorithm 10.7 It allows us to combine the ideals describing certain loci in the individual schemes $\mathbb{B}_{\mathcal{O}}^{\text {dfb }}\left(\mathcal{O}^{\prime}\right)$ to ideals describing them in $\mathbb{B}_{\mathcal{O}}(\mathcal{H})$, and the underlying base changes and morphisms of universal families are made explicit. Some computational simplifications are suggested in Remark 10.9.

The last three Sections 11, 12, and 13 apply the technique of Algorithm 10.7 and the respective methods to the degree filtered case. In Section 11, we compute the Cayley-Bacharach locus of $\mathbb{B}_{\mathcal{O}}(\mathcal{H})$ (see Algorithm 11.1) and derive a method for calculating the strict Gorenstein locus (see Corollary 11.4). Next, Section 12 treats the strict Cayley-Bacharach locus of $\mathbb{B}_{\mathcal{O}}(\mathcal{H})$ via Algorithm 12.1, and Section 13 shows how to compute the strict complete intersection locus in $\mathbb{B}_{\mathcal{O}}(\mathcal{H})$ via 


\section{Algorithm 13.1.}

All algorithms are illustrated by applying them to non-trivial, explicit examples. They show that one can "really do it" in small cases, rather than being able to "do it in principle". These examples were calculated by a package for the computer algebra system $\mathrm{CoCoA}$ (see [1) written by the second author, and available via the project web page (see [16]).

Before starting with the paper properly, let us mention some general aspects of the results and their presentation. We always work over an arbitrary base field $K$. This entails that all results are characteristic-free. Although we shall frequently talk about $K$-rational points of certain schemes, such points may not exist over the given field. Notice that we may enlarge $K$ without changing the results of the computations, because they are performed over $K$. Hence the claims remain true if we consider all $L$-rational points for a field extension $L \supseteq K$, for instance, the algebraic closure $L=\bar{K}$, and any mention of $K$-rational points should be read in this way.

In principle, all subschemes we consider should be equipped with the induced reduced scheme structure. However, our algorithms produce ideals defining the desired subsets of the border basis scheme which are not necessarily radical ideals. Of course, if we replace one ideal $I$ by another ideal $J$ such that $\operatorname{Rad}(I)=\operatorname{Rad}(J)$, then the ideal $J$ defines the same set of $K$-rational points as $I$. To simplify the exposition and the calculations, when we write that an ideal "defines a subscheme", we really mean "up to radical". On a number of occasions, this freedom will be essential, since the calculation of the radical of an ideal in many indeterminates can be an unsurmountable burden.

Unless explicitly stated otherwise, we use the notation and definitions of [17, [18], and [19.

\section{Border Bases of Zero-Dimensional Ideals}

In the following we let $K$ be a field, let $P=K\left[x_{1}, \ldots, x_{n}\right]$ be a polynomial ring over $K$, let $\mathbb{T}^{n}=\left\{x_{1}^{\alpha_{1}} \cdots x_{n}^{\alpha_{n}} \mid \alpha_{i} \geq 0\right\}$ be the monoid of terms in $P$, and let $I$ be a 0 -dimensional ideal in $P$. Border bases of $I$ are defined as follows.

Definition 2.1. Let $\mu \geq 1$.

(a) A set of terms $\mathcal{O}=\left\{t_{1}, \ldots, t_{\mu}\right\}$ is called an order ideal in $\mathbb{T}^{n}$ if $t \in \mathcal{O}$ implies that every term $t^{\prime} \in \mathbb{T}^{n}$ which divides $t$ is also contained in $\mathcal{O}$.

(b) For an order ideal $\mathcal{O}$, the set of terms $\partial \mathcal{O}=\left(x_{1} \mathcal{O} \cup \cdots \cup x_{n} \mathcal{O}\right) \backslash \mathcal{O}$ is called the border of $\mathcal{O}$.

(c) Let $\mathcal{O}=\left\{t_{1}, \ldots, t_{\mu}\right\}$ be an order ideal and $\partial \mathcal{O}=\left\{b_{1}, \ldots, b_{\nu}\right\}$ its border. A set of polynomials $G=\left\{g_{1}, \ldots, g_{\nu}\right\}$ in $P$ is called an $\mathcal{O}$-border prebasis if they are of the form $g_{j}=b_{j}-\sum_{i=1}^{\mu} \gamma_{i j} t_{i}$ with $\gamma_{i j} \in K$ for $i=1, \ldots, \mu$ and $j=1, \ldots, \nu$. 
(d) An $\mathcal{O}$-border prebasis $G \subset I$ is called an $\mathcal{O}$-border basis of $I$ if the residue classes of the terms in $\mathcal{O}$ from a $K$-basis of $P / I$.

For more information about border bases, we refer to [18, Section 6.4. In particular, we note that a border basis always generates the ideal $I$.

Assumption 2.2. For an order ideal $\mathcal{O}=\left\{t_{1}, \ldots, t_{\mu}\right\}$ in $\mathbb{T}^{n}$, we always assume that $\operatorname{deg}\left(t_{1}\right) \leq \cdots \leq \operatorname{deg}\left(t_{\mu}\right)$. In particular, this implies that we have $t_{1}=1$.

Another way to view this setting is given by Algebraic Geometry. Here we consider the 0 -dimensional subscheme $\mathbb{X}$ of $\mathbb{A}_{K}^{n}$ whose vanishing ideal is $I$. We denote its affine coordinate ring $P / I$ by $R_{\mathbb{X}}$ and write $I_{\mathbb{X}}$ instead of $I$. Notice that we always choose a fixed embedding of $\mathbb{X}$ into $\mathbb{A}_{K}^{n}=\operatorname{Spec}(P)$ and also fix the coordinate system. Clearly, the notion of a border basis of $I_{\mathbb{X}}$ depends on these choices. The vector space dimension of $R_{\mathbb{X}}$ over $K$ is finite. It is sometimes called the length of $\mathbb{X}$ and will be denoted by $\mu=\operatorname{dim}_{K}\left(R_{\mathbb{X}}\right)$.

Since we are keeping the coordinate system fixed at all times, we have further invariants of $\mathbb{X}$. Recall that the degree filtration $\widetilde{\mathcal{F}}=\left(F_{i} P\right)_{i \in \mathbb{Z}}$ on $P$ is given by $F_{i} P=\{f \in P \backslash\{0\} \mid \operatorname{deg}(f) \leq i\} \cup\{0\}$ for all $i \in \mathbb{Z}$. The induced filtration $\mathcal{F}=\left(F_{i} R_{\mathbb{X}}\right)_{i \in \mathbb{Z}}$, where $F_{i} R_{\mathbb{X}}=F_{i} P /\left(F_{i} P \cap I_{\mathbb{X}}\right)$, is called the degree filtration on $R_{\mathbb{X}}$. The degree filtration on $R_{\mathbb{X}}$ is increasing, exhaustive and orderly in the sense that every element $\bar{f} \in R_{\mathbb{X}} \backslash\{0\}$ has an order

$$
\operatorname{ord}_{\mathcal{F}}(\bar{f})=\min \left\{i \in \mathbb{Z} \mid \bar{f} \in F_{i} R_{\mathbb{X}} \backslash F_{i-1} R_{\mathbb{X}}\right\}
$$

The degree filtration allows us to introduce the following concepts.

Definition 2.3. Let $\mathbb{X}$ be a 0 -dimensional subscheme of $\mathbb{A}_{K}^{n}$ of length $\mu$.

(a) The map $\operatorname{HF}_{\mathbb{X}}^{a}: \mathbb{Z} \longrightarrow \mathbb{Z}$ given by $i \mapsto \operatorname{dim}_{K}\left(F_{i} R_{\mathbb{X}}\right)$ is called the affine Hilbert function of $\mathbb{X}$. It is a monotonously increasing function which satisfies $\mathrm{HF}_{\mathbb{X}}^{a}(i)=\mu$ for $i \gg 0$. Here the number

$$
\operatorname{ri}\left(R_{\mathbb{X}}\right)=\min \left\{i \in \mathbb{Z} \mid \operatorname{HF}_{\mathbb{X}}^{a}(j)=\mu \text { for all } j \geq i\right\}
$$

is called the regularity index of $\mathbb{X}$.

(b) The first difference function $\Delta \mathrm{HF}_{\mathbb{X}}^{a}(i)=\mathrm{HF}_{\mathbb{X}}^{a}(i)-\mathrm{HF}_{\mathbb{X}}^{a}(i-1)$ of $\mathrm{HF}_{\mathbb{X}}^{a}$ is called the Castelnuovo function of $\mathbb{X}$, and $\Delta_{\mathbb{X}}=\Delta \operatorname{HF}_{\mathbb{X}}^{a}\left(\operatorname{ri}\left(R_{\mathbb{X}}\right)\right)$ is called the last difference of $\mathbb{X}$.

(c) Given an order ideal $\mathcal{O}=\left\{t_{1}, \ldots, t_{\mu}\right\}$ in $\mathbb{T}^{n}$ and a number $i \geq 0$, we let $h_{i}=\#\left\{j \in\{1, \ldots, \mu\} \mid \operatorname{deg}\left(t_{j}\right)=i\right\}$. Then $\operatorname{HF}_{\mathcal{O}}=\left(h_{0}, h_{1}, \ldots\right)$ is called the Hilbert function of $\mathcal{O}$, and $\mathrm{HF}_{\mathcal{O}}^{a}=\left(h_{0}, h_{0}+h_{1}, h_{0}+h_{1}+h_{2}, \ldots\right)$ is called the affine Hilbert function of $\mathcal{O}$. In other words, we have $\mathrm{HF}_{\mathcal{O}}=\Delta \mathrm{HF}_{\mathcal{O}}^{a}$.

The affine Hilbert function of $\mathbb{X}$ satisfies $\operatorname{HF}_{\mathbb{X}}^{a}(i)=0$ for $i<0$ and

$$
1=\operatorname{HF}_{\mathbb{X}}^{a}(0)<\operatorname{HF}_{\mathbb{X}}^{a}(1)<\cdots<\operatorname{HF}_{\mathbb{X}}^{a}\left(\operatorname{ri}\left(R_{\mathbb{X}}\right)\right)=\mu
$$


as well as $\operatorname{HF}_{\mathbb{X}}^{a}(i)=\mu$ for $i \geq \operatorname{ri}\left(R_{\mathbb{X}}\right)$. Notice that, in general, the affine Hilbert function of $\mathcal{O}$ differs from the affine Hilbert function of $\mathbb{X}$, even if $\mathcal{O}$ represents a $K$-basis of $R_{\mathbb{X}}$. We will come back to this point later (see Section 5 ).

One application of the degree filtration on $R_{\mathbb{X}}$ is the possibility of passing to the degree forms of polynomials and to reduce many considerations to the homogeneous case. Recall that the degree form of a polynomial $f \in P \backslash\{0\}$ is its homogeneous component of highest degree and is denoted by $\operatorname{DF}(f)$. Given an ideal $I \subseteq P$, we let $\operatorname{DF}(I)=\langle\operatorname{DF}(f) \mid f \in I \backslash\{0\}\rangle$ be the degree form ideal of $I$. Passage to the $\operatorname{ring} R_{\mathbb{X}}$ and the induced filtration $\mathcal{F}$ leads to the following notions.

Definition 2.4. Let $\mathbb{X}$ be a 0-dimensional subscheme of $\mathbb{A}_{K}^{n}$.

(a) For an element $f \in R_{\mathbb{X}} \backslash\{0\}$ of order $d=\operatorname{ord}_{\mathcal{F}}(f)$, the residue class $\operatorname{LF}(f)=$ $f+F_{d-1} R_{\mathbb{X}}$ in $\operatorname{gr}_{\mathcal{F}}\left(R_{\mathbb{X}}\right)$ is called the leading form of $f$ with respect to $\mathcal{F}$.

(b) The ring $\operatorname{gr}_{\mathcal{F}}\left(R_{\mathbb{X}}\right)=\bigoplus_{i \in \mathbb{Z}} F_{i} R_{\mathbb{X}} / F_{i-1} R_{\mathbb{X}}$ is called the associated graded ring of $R_{\mathbb{X}}$ with respect to $\mathcal{F}$.

In our setting the associated graded $\operatorname{ring} \operatorname{gr}_{\mathcal{F}}\left(R_{\mathbb{X}}\right)$ is a 0 -dimensional local ring with maximal ideal $\left\langle\bar{x}_{1}, \ldots, \bar{x}_{n}\right\rangle$. Its $K$-vector space dimension is given by $\operatorname{dim}_{K}\left(\operatorname{gr}_{\mathcal{F}}\left(R_{\mathbb{X}}\right)\right)=\sum_{i=0}^{\infty} \operatorname{dim}_{K}\left(F_{i} R_{\mathbb{X}} / F_{i-1} R_{\mathbb{X}}\right)=\operatorname{dim}_{K}\left(R_{\mathbb{X}}\right)$. Every non-zero homogeneous element of $\operatorname{gr}_{\mathcal{F}}\left(R_{\mathbb{X}}\right)$ is of the form $\operatorname{LF}(f)$ for some $f \in R_{\mathbb{X}} \backslash\{0\}$. For the algorithms in the later sections, the most important property of the associated graded ring is that it can be computed explicitly via the formula $\operatorname{gr}_{\mathcal{F}}\left(R_{\mathbb{X}}\right) \cong P / \operatorname{DF}\left(I_{\mathbb{X}}\right)$.

\section{The Border Basis Scheme}

In the following we let $K$ be a field, and we let $\mathcal{O}=\left\{t_{1}, \ldots, t_{\mu}\right\}$ be an order ideal in $\mathbb{T}^{n}$. The $\mathcal{O}$-border basis scheme is a moduli scheme which parametrizes all 0 -dimensional ideals having an $\mathcal{O}$-border basis. Let us recall its definition and its defining ideal.

Definition 3.1. Let $\mathcal{O}=\left\{t_{1}, \ldots, t_{\mu}\right\}$ be an order ideal, and let $\partial \mathcal{O}=\left\{b_{1}, \ldots, b_{\nu}\right\}$ be the border of $\mathcal{O}$.

(a) Let $C=\left\{c_{i j} \mid i \in\{1, \ldots, \mu\}, j \in\{1, \ldots, \nu\}\right\}$ be a set of new indeterminates, and let $K[C]=K\left[c_{11}, \ldots, c_{\mu \nu}\right]$. Then the set of polynomials $G=\left\{g_{1}, \ldots, g_{\nu}\right\}$ in $K[C]\left[x_{1}, \ldots, x_{n}\right]$, where

$$
g_{j}=b_{j}-c_{1 j} t_{1}-\cdots-c_{\mu j} t_{\mu}
$$

for $j=1, \ldots, \nu$, is called the generic $\mathcal{O}$-border prebasis.

(b) For $r=1, \ldots, n$, the matrix $\mathcal{A}_{r}=\left(a_{i j}^{(r)}\right) \in \operatorname{Mat}_{\mu}(K[C])$, where $K[C]=$ $K\left[c_{11}, \ldots, c_{\mu \nu}\right]$ and

$$
a_{i j}^{(r)}= \begin{cases}\delta_{i m} & \text { if } x_{r} t_{j}=t_{m} \\ c_{i m} & \text { if } x_{r} t_{j}=b_{m}\end{cases}
$$

is called the $r$-th generic multiplication matrix for $\mathcal{O}$. 
(c) Consider the ideal in $K[C]$ which is generated by all entries of the commutator matrices $\mathcal{A}_{r} \mathcal{A}_{s}-\mathcal{A}_{s} \mathcal{A}_{r}$ with $1 \leq r<s \leq n$. Then the subscheme of $\mathbb{A}_{K}^{\mu \nu}=$ $\operatorname{Spec}(K[C])$ defined by this ideal is called the $\mathcal{O}$-border basis scheme. It is denoted by $\mathbb{B}_{\mathcal{O}}$, the defining ideal is denoted by $I\left(\mathbb{B}_{\mathcal{O}}\right)$, and the corresponding affine coordinate ring is denoted by $B_{\mathcal{O}}=K[C] / I\left(\mathbb{B}_{\mathcal{O}}\right)$.

(d) The ring homomorphism

$$
B_{\mathcal{O}} \longrightarrow U_{\mathcal{O}}:=B_{\mathcal{O}}\left[x_{1}, \ldots, x_{n}\right] /\left\langle g_{1}, \ldots, g_{\nu}\right\rangle
$$

is called the universal $\mathcal{O}$-border basis family.

The main reason why $\mathbb{B}_{\mathcal{O}}$ is a good moduli space is the following result (see [20, Theorem 3.4).

Theorem 3.2. The residue classes of the elements of $\mathcal{O}$ are a $B_{\mathcal{O}}$-basis of $U_{\mathcal{O}}$.

Thus the universal $\mathcal{O}$-border basis family is flat and parametrizes all $\mathcal{O}$-border bases. Its $K$-rational points correspond to $\mathcal{O}$-border bases in the following way.

Definition 3.3. Let $\Gamma=\left(\gamma_{i j}\right) \in K^{\mu \nu}$ be a $K$-rational point of $\mathbb{B}_{\mathcal{O}}$. Then the polynomials $g_{j}\left(x_{1}, \ldots, x_{n}, \gamma_{11}, \ldots, \gamma_{\mu \nu}\right)$ with $j \in\{1, \ldots, \nu\}$ form an $\mathcal{O}$-border basis. Let $I_{\Gamma}$ be the ideal in $P$ which is generated by these polynomials. Then the 0 -dimensional scheme $\mathbb{X}_{\Gamma}$ in $\mathbb{A}_{K}^{n}$ defined by $I_{\Gamma}$ is called the 0 -dimensional scheme represented by $\Gamma$.

Conversely, given a 0 -dimensional scheme $\mathbb{X}$ in $\mathbb{A}_{K}^{n}$ whose vanishing ideal $I_{\mathbb{X}}$ has an $\mathcal{O}$-border basis, the coefficients of that $\mathcal{O}$-border basis define a $K$-rational point $\Gamma_{\mathbb{X}}$ of $\mathbb{B}_{\mathcal{O}}$. We say that the point $\Gamma_{\mathbb{X}}$ represents the 0 -dimensional scheme $\mathbb{X}$ in $\mathbb{B}_{\mathcal{O}}$.

Using this terminology, our goal in the next sections is to describe the subsets of $\mathbb{B}_{\mathcal{O}}$ whose $K$-rational points represent the 0 -dimensional schemes that are locally Gorenstein, strict Gorenstein schemes, Cayley-Bacharach and strict CayleyBacharach schemes, or strict complete intersections. Notice that these subsets will frequently be neither the set of $K$-rational points of an open nor of a closed set in the Zariski topology, but merely the set of $K$-rational points of a constructible subset of $\mathbb{B}_{\mathcal{O}}$. As the case may be, we shall try to describe their structure as explicitly as possible.

An important property of $I\left(\mathbb{B}_{\mathcal{O}}\right)$ is that it is homogeneous with respect to the following grading.

Definition 3.4. Let $\mathcal{O}=\left\{t_{1}, \ldots, t_{\mu}\right\}$ be an order ideal, let $\partial \mathcal{O}=\left\{b_{1}, \ldots, b_{\nu}\right\}$ be the border of $\mathcal{O}$, and let $C=\left\{c_{i j} \mid i \in\{1, \ldots, \mu\}, j \in\{1, \ldots, \nu\}\right\}$ be the set of indeterminates representing the coefficients of the generic $\mathcal{O}$-border prebasis. Then the $\mathbb{Z}$-grading on $K[C]$ defined by $\operatorname{deg}\left(c_{i j}\right)=\operatorname{deg}\left(b_{j}\right)-\operatorname{deg}\left(t_{i}\right)$ for $i=1, \ldots, \mu$ and $j=1, \ldots, \nu$ is called the total arrow degree. 
The name of this grading derives from the fact that we may view $c_{i j}$ as an arrow pointing from $b_{j}$ to $t_{i}$ as in [10, p. 210 and 12, Section 3. The next proposition shows why the total arrow degree is useful for us.

Proposition 3.5. The ideal $I\left(\mathbb{B}_{\mathcal{O}}\right)$ in $K[C]$ defining the border basis scheme is homogeneous with respect to the total arrow degree.

Proof. First we note that the generic $\mathcal{O}$-border prebasis $G$ is homogeneous with respect to the total arrow degree, if we let $\operatorname{deg}\left(x_{i}\right)=1$ for $i=1, \ldots, n$, as usual. Hence the universal family $U_{\mathcal{O}}=K[C]\left[x_{1}, \ldots, x_{n}\right] /\langle G\rangle$ is a graded ring with respect to this grading. Now we observe that $\mathcal{O}$ is a homogeneous $B_{\mathcal{O}}$ basis of $U_{\mathcal{O}}$. For $i=1, \ldots, n$, the generic multiplication matrix $\mathcal{A}_{i}$ expresses the multiplication by $x_{i}$ in this basis. Thus it yields a homogeneous $B_{\mathcal{O}}$-linear map $\mu_{x_{i}}: \bigoplus_{j=1}^{\mu} B_{\mathcal{O}}\left(-\operatorname{deg}\left(t_{j}\right)-1\right) \longrightarrow \bigoplus_{j=1}^{\mu} B_{\mathcal{O}}\left(-\operatorname{deg}\left(t_{j}\right)\right)$ of degree zero. Consequently, the commutators $\mathcal{A}_{k} \mathcal{A}_{\ell}-\mathcal{A}_{\ell} \mathcal{A}_{k}$ are homogeneous $B_{\mathcal{O}}$-linear maps of degree zero, and their entries are homogeneous polynomials with respect to the total arrow degree, as was to be shown.

In 23], Prop. 3.2.6, it is shown that $I\left(\mathbb{B}_{\mathcal{O}}\right)$ is even homogeneous with respect to the $\mathbb{Z}^{n}$-grading $\operatorname{deg}\left(c_{i j}\right)=\log \left(b_{j}\right)-\log \left(t_{i}\right)$ which is called the arrow grading. This yields another proof for the above proposition. Let us check it in a concrete case.

Example 3.6. Let $K$ be a field, let $P=K[x, y]$, and let $\mathcal{O}=\{1, x, y, x y\}$. Then the border of $\mathcal{O}$ is given by $\partial \mathcal{O}=\left\{x^{2}, y^{2}, x^{2} y, x y^{2}\right\}$. The generic multiplication matrices are

$$
\mathcal{A}_{x}=\left(\begin{array}{llll}
0 & c_{11} & 0 & c_{13} \\
1 & c_{21} & 0 & c_{23} \\
0 & c_{31} & 0 & c_{33} \\
0 & c_{41} & 1 & c_{43}
\end{array}\right) \quad \text { and } \quad \mathcal{A}_{y}=\left(\begin{array}{ccccc}
0 & 0 & c_{12} & c_{14} \\
0 & 0 & c_{22} & c_{24} \\
1 & 0 & c_{32} & c_{34} \\
0 & 1 & c_{42} & c_{44}
\end{array}\right)
$$

Consequently, the defining ideal $I\left(\mathbb{B}_{\mathcal{O}}\right)$ of the border basis scheme $\mathbb{B}_{\mathcal{O}}$ is generated by the entries of $\mathcal{A}_{x} \mathcal{A}_{y}-\mathcal{A}_{y} \mathcal{A}_{x}$, i.e., by the polynomials

$$
\begin{array}{ll}
\left\{c_{11} c_{22}+c_{13} c_{42}-c_{14},\right. & c_{11} c_{24}-c_{12} c_{33}-c_{14} c_{43}+c_{13} c_{44}, \\
c_{12} c_{31}+c_{14} c_{41}-c_{13}, & c_{21} c_{22}+c_{23} c_{42}+c_{12}-c_{24}, \\
c_{21} c_{24}-c_{22} c_{33}-c_{24} c_{43}+c_{23} c_{44}+c_{14}, & c_{22} c_{31}+c_{24} c_{41}-c_{23}, \\
c_{22} c_{31}+c_{33} c_{42}-c_{34}, & c_{22} c_{41}+c_{42} c_{43}+c_{32}-c_{44}, \\
c_{24} c_{31}-c_{32} c_{33}-c_{34} c_{43}+c_{33} c_{44}-c_{13}, & c_{31} c_{32}+c_{34} c_{41}+c_{11}-c_{33}, \\
c_{31} c_{42}+c_{41} c_{44}+c_{21}-c_{43}, & \left.c_{33} c_{42}-c_{24} c_{41}+c_{23}-c_{34}\right\}
\end{array}
$$

The degree tuple of the total arrow degree is

$$
\left(\operatorname{deg}\left(c_{11}\right), \operatorname{deg}\left(c_{12}\right), \ldots, \operatorname{deg}\left(c_{44}\right)\right)=(2,2,3,3,1,1,2,2,1,1,2,2,0,0,1,1)
$$

and it is easy to verify that the above polynomials are indeed homogeneous with respect to the grading it defines. As shown in [20, Example 3.8, the scheme $\mathbb{B}_{\mathcal{O}}$ is isomorphic to an 8-dimensional affine space over $K$. 


\section{The Locally Gorenstein Locus}

The first subscheme of the border basis scheme which we want to describe explicitly is the open subscheme parametrizing 0-dimensional locally Gorenstein schemes. Recall that a local ring $S$ is said to be Gorenstein if its socle, i.e., the annihilator of its maximal ideal $\mathfrak{n}$ is a 1 -dimensional $S / \mathfrak{n}$-vector space. For equivalent definitions, see for instance [6], Ch. 21.

Definition 4.1. Let $\mathbb{X}$ be a 0 -dimensional subscheme of $\mathbb{A}_{K}^{n}$, let $I_{\mathbb{X}}=\mathfrak{Q}_{1} \cap \cdots \cap \mathfrak{Q}_{s}$ be the primary decomposition of its vanishing ideal in $P$, and let $\mathfrak{q}_{i}$ be the image of $\mathfrak{Q}_{i}$ in $R_{\mathbb{X}}=P / I_{\mathbb{X}}$ for $i=1, \ldots, s$.

(a) The ring $R_{\mathbb{X}}$ is called a locally Gorenstein ring if $R_{\mathbb{X}} / \mathfrak{q}_{i}$ is a local Gorenstein ring for $i=1, \ldots, s$.

(b) The scheme $\mathbb{X}$ is said to be locally Gorenstein if $R_{\mathbb{X}}$ is a locally Gorenstein ring.

Sometimes locally Gorenstein schemes are simply called Gorenstein, if no confusion can arise. An algorithm for checking the locally Gorenstein property was given in [14, Alg. 5.4.

Now we let $\mathcal{O}=\left\{t_{1}, \ldots, t_{\mu}\right\}$ be an order ideal in $\mathbb{T}^{n}$. Our goal is to describe the following subset of the border basis scheme $\mathbb{B}_{\mathcal{O}}$.

Definition 4.2. The set of all $K$-rational points $\Gamma=\left(\gamma_{i j}\right) \in K^{\mu \nu}$ of the border basis scheme $\mathbb{B}_{\mathcal{O}}$ which represent a locally Gorenstein 0-dimensional scheme $\mathbb{X}_{\Gamma}$ is called the set of locally Gorenstein points of $\mathbb{B}_{\mathcal{O}}$.

Based on Algorithm 5.4 in [14, we can describe the set of locally Gorenstein points of $\mathbb{B}_{\mathcal{O}}$. As the following algorithm shows, it is the set of $K$-rational points of an open subscheme $\operatorname{LGor}_{\mathcal{O}}$ of $\mathbb{B}_{\mathcal{O}}$ which we call the locally Gorenstein locus in $\mathbb{B}_{\mathcal{O}}$.

Algorithm 4.3. (Computing the Locally Gorenstein Locus)

Let $\mathcal{O}=\left\{t_{1}, \ldots, t_{\mu}\right\}$ be an order ideal in $\mathbb{T}^{n}$. Consider the following sequence of instructions.

(1) Determine the generic multiplication matrices $\mathcal{A}_{1}, \ldots, \mathcal{A}_{n}$ for $\mathcal{O}$ in the ring $\operatorname{Mat}_{\mu}(K[C])$.

(2) Calculate the commutators $\mathcal{A}_{r} \mathcal{A}_{s}-\mathcal{A}_{s} \mathcal{A}_{r}$ for $1 \leq r<s \leq n$ and form the ideal $I\left(\mathbb{B}_{\mathcal{O}}\right)$ in $K[C]$ generated by their entries.

(3) Introduce new indeterminates $z_{1}, \ldots, z_{\mu}$ and construct the matrix $D$ in $\operatorname{Mat}_{\mu}\left(K[C]\left[z_{1}, \ldots, z_{\mu}\right]\right)$ whose $i$-th column is given by

$$
t_{i}\left(\mathcal{A}_{1}^{\mathrm{tr}}, \ldots, \mathcal{A}_{n}^{\mathrm{tr}}\right) \cdot\left(z_{1}, \ldots, z_{\mu}\right)^{\operatorname{tr}}
$$

for $i=1, \ldots, \mu$.

(4) Compute $\operatorname{det}(D)$ in $K[C]\left[z_{1}, \ldots, z_{\mu}\right]$, and let $J$ be the ideal in $K[C]$ generated

by the coefficients of $\operatorname{det}(D)$ with respect to the indeterminates $z_{1}, \ldots, z_{\mu}$.

(5) Return the ideal $I\left(\mathbb{B}_{\mathcal{O}}\right)+J$. 
This is an algorithm which computes an ideal in $K[C]$ which defines a closed subscheme NonLGor $\mathrm{O}_{\mathcal{O}}$ of $\mathbb{B}_{\mathcal{O}}$ such that the set of $K$-rational points of the complement $\mathbb{B}_{\mathcal{O}} \backslash$ NonLGor $_{\mathcal{O}}$ is precisely the set of locally Gorenstein points of $\mathbb{B}_{\mathcal{O}}$.

Proof. Let us apply [14, Alg. 5.4, where we use the basis $B=\left(\bar{t}_{1}, \ldots, \bar{t}_{\mu}\right)$ of $R_{\mathbb{X}}$. For a fixed $K$-rational point $\Gamma=\left(\gamma_{i j}\right)$ of $\mathbb{B}_{\mathcal{O}}$, the corresponding ideal $I_{\Gamma}$ defines a locally Gorenstein scheme if and only if the polynomial obtained by substituting $\left(\gamma_{i j}\right)$ for $\left(c_{i j}\right)$ in $\operatorname{det}(D)$ is non-zero. Thus the non-locally Gorenstein locus is defined by equating all coefficients of $\operatorname{det}(D)$ to zero, and the claim follows.

In view of this algorithm we see that the locally Gorenstein locus LGor $_{\mathcal{O}}=$ $\mathbb{B}_{\mathcal{O}} \backslash$ NonLGor $_{\mathcal{O}}$ is an open subscheme of $\mathbb{B}_{\mathcal{O}}$. Let us compute this locus in the setting of Example 3.6 .

Example 4.4. For the order ideal $\mathcal{O}=\{1, x, y, x y\}$, let us compute the ideal defining the non-locally Gorenstein locus in $\mathbb{B}_{\mathcal{O}}$. The ideal $I\left(\mathbb{B}_{\mathcal{O}}\right)$ was computed in Example 3.6. Let $\mathcal{Z}=\left(z_{1}, z_{2}, z_{3}, z_{4}\right)^{\text {tr }}$, and let $D$ be the matrix $D=\left(\mathcal{Z}, \mathcal{A}_{x}^{\operatorname{tr}} \mathcal{Z}, \mathcal{A}_{y}^{\operatorname{tr}} \mathcal{Z}, \mathcal{A}_{x}^{\operatorname{tr}} \mathcal{A}_{y}^{\operatorname{tr}} \mathcal{Z}\right)$. Its four columns are

$$
\mathcal{Z}, \quad\left(\begin{array}{llll}
0 & 1 & 0 & 0 \\
c_{11} & c_{21} & c_{31} & c_{41} \\
0 & 0 & 0 & 1 \\
c_{13} & c_{23} & c_{33} & c_{43}
\end{array}\right) \mathcal{Z}, \quad\left(\begin{array}{llll}
0 & 0 & 1 & 0 \\
0 & 0 & 0 & 1 \\
c_{12} & c_{22} & c_{32} & c_{42} \\
c_{14} & c_{24} & c_{34} & c_{44}
\end{array}\right) \mathcal{Z}, \quad\left(\begin{array}{llll}
0 & 0 & 0 & 1 \\
p_{1} & p_{2} & p_{3} & p_{4} \\
c_{14} & c_{24} & c_{34} & c_{44} \\
q_{1} & q_{2} & q_{3} & q_{4}
\end{array}\right) \mathcal{Z}
$$

where $p_{1}=c_{12} c_{31}+c_{14} c_{41}, p_{2}=c_{22} c_{31}+c_{24} c_{41}, p_{3}=c_{31} c_{32}+c_{34} c_{41}+c_{11}$, $p_{4}=c_{31} c_{42}+c_{41} c_{44}+c_{21}, q_{1}=c_{12} c_{33}+c_{14} c_{43}, q_{2}=c_{22} c_{33}+c_{24} c_{43}, q_{3}=c_{32} c_{33}+$ $c_{34} c_{43}+c_{13}$, and $q_{4}=c_{33} c_{42}+c_{43} c_{44}+c_{23}$.

The determinant of $D$ is a polynomial

$$
\begin{aligned}
\operatorname{det}(D)= & \left(-c_{12}^{2} c_{13} c_{31}+c_{11} c_{12}^{2} c_{33}-c_{12} c_{13} c_{14} c_{41}+c_{11} c_{12} c_{14} c_{43}-c_{11} c_{14}^{2}\right) z_{1}^{4}+\cdots \\
& \cdots+\left(-c_{41} c_{42}+1\right) z_{4}^{4}
\end{aligned}
$$

in $K[C]\left[z_{1}, z_{2}, z_{3}, z_{4}\right]$ which is homogeneous of degree 4 with respect to $z_{1}, \ldots, z_{4}$ and has 35 non-zero coefficients in $K[C]$. Let $J$ be the ideal generated by these coefficients. Then the set NonLGor $(\mathcal{O})$ is defined by the ideal $I\left(\mathbb{B}_{\mathcal{O}}\right)+J$.

Here we can compute a Gröbner basis of $I\left(\mathbb{B}_{\mathcal{O}}\right)+J$ and check that we have $\operatorname{dim}\left(K[C] /\left(I\left(\mathbb{B}_{\mathcal{O}}\right)+J\right)\right)=4$. Hence $\operatorname{NonLGor}(\mathcal{O})$ is the set of closed points of a 4 -dimensional closed subscheme of the 8-dimensional scheme $\mathbb{B}_{\mathcal{O}}$.

\section{The Degree Filtered Border Basis Scheme $\mathbb{B}_{\mathcal{O}}^{\text {df }}$}

As before, we let $K$ be a field, $P=K\left[x_{1}, \ldots, x_{n}\right]$, and $\mathcal{O}=\left\{t_{1}, \ldots, t_{\mu}\right\}$ an order ideal in $\mathbb{T}^{n}$ with border $\partial \mathcal{O}=\left\{b_{1}, \ldots, b_{\nu}\right\}$. In this section we focus on a subscheme of the border basis scheme $\mathbb{B}_{\mathcal{O}}$ which parametrizes those schemes $\mathbb{X}$ for which $\mathcal{O}$ yields a particularly nice $K$-basis of $R_{\mathbb{X}}$. More precisely, recall that the degree filtration $\left(F_{i} P\right)_{i \in \mathbb{Z}}$ on $P$ is defined by $F_{i} P=\{f \in P \mid \operatorname{deg}(f) \leq i\} \cup\{0\}$. For 
every $i \in \mathbb{Z}$, let $F_{i} I=F_{i} P \cap I$, and let $F_{i} R=F_{i} P / F_{i} I$. Then the family $\left(F_{i} I\right)_{i \in \mathbb{Z}}$ is called the induced filtration on $I$, and the family $\mathcal{F}=\left(F_{i} R\right)_{i \in \mathbb{Z}}$ is a $\mathbb{Z}$-filtration on $R$ which is called the degree filtration on $R$. For more details on filtrations, see for instance [14] and Section 6.5 of [18].

Definition 5.1. Let $\mathbb{X}$ be a 0 -dimensional subscheme of $\mathbb{A}_{K}^{n}$ with vanishing ideal $I_{\mathbb{X}} \subseteq P$ and affine coordinate ring $R_{\mathbb{X}}=P / I_{\mathbb{X}}$.

(a) A tuple $B=\left(\bar{t}_{1}, \ldots, \bar{t}_{\mu}\right) \in R_{\mathbb{X}}^{\mu}$ is called a degree filtered $K$-basis of $R_{\mathbb{X}}$ if the set $F_{i} B=B \cap F_{i} R_{\mathbb{X}}$ is a $K$-basis of $F_{i} R_{\mathbb{X}}$ for every $i \in \mathbb{Z}$ and if $\operatorname{ord}_{\mathcal{F}}\left(\bar{t}_{1}\right) \leq \cdots \leq \operatorname{ord}_{\mathcal{F}}\left(\bar{t}_{\mu}\right)$.

(b) Given an order ideal $\mathcal{O}=\left\{t_{1}, \ldots, t_{\mu}\right\}$ in $\mathbb{T}^{n}$, we say that $\mathbb{X}$ has a degree filtered $\mathcal{O}$-border basis if the tuple of residue classes $\left(\bar{t}_{1}, \ldots, \bar{t}_{\mu}\right)$ is a degree filtered $K$-basis of $R_{\mathbb{X}}$.

For the sake of completeness, the following example borrowed from [14 provides a monomial $K$-basis which is not degree-filtered.

Example 5.2. Let $K=\mathbb{Q}$, let $P=K[x, y]$, let $I$ be the vanishing ideal of the affine set of eight points given by $p_{1}=(1,-1), p_{2}=(0,2), p_{3}=(1,1), p_{4}=(1,2)$, $p_{5}=(0,1), p_{6}=(1,3), p_{7}=(2,4)$, and $p_{8}=(3,4)$, and let $R=P / I$. The reduced Gröbner basis of $I$ with respect to DegRevLex is

$$
\begin{gathered}
\left\{x^{2} y-4 x^{2}-x y+4 x, \quad x^{3}+x y^{2}-6 x^{2}-3 x y-y^{2}+7 x+3 y-2,\right. \\
y^{4}-10 x y^{2}-5 y^{3}+15 x^{2}+30 x y+15 y^{2}-35 x-25 y+14 \\
\left.x y^{3}-7 x y^{2}-y^{3}+14 x y+7 y^{2}-8 x-14 y+8\right\}
\end{gathered}
$$

Since this term ordering is degree compatible, the residue classes of the elements in the tuple $\left(1, y, x, y^{2}, x y, x^{2}, y^{3}, x y^{2}\right)$ form a degree-filtered $K$-basis of $R$ with order tuple $(0,1,1,2,2,2,3,3)$. On the other hand, the reduced Gröbner basis of $I$ with respect to Lex is

$$
\begin{gathered}
\left\{x^{2}-\frac{2}{3} x y^{2}+2 x y-\frac{7}{3} x+\frac{1}{15} y^{4}-\frac{1}{3} y^{3}+y^{2}-\frac{5}{3} y+\frac{14}{15},\right. \\
\left.x y^{3}-7 x y^{2}+14 x y-8 x-y^{3}+7 y^{2}-14 y+8, y^{5}-9 y^{4}+25 y^{3}-15 y^{2}-26 y+24\right\}
\end{gathered}
$$

So, the residue classes of the elements in the tuple $B=\left(1, y, x, y^{2}, x y, y^{3}, x y^{2}, y^{4}\right)$ form a $K$-basis of $R$. Since $\bar{y}^{4}=10 \bar{x} \bar{y}^{2}+5 \bar{y}^{3}-15 \bar{x}^{2}-30 \bar{x} \bar{y}-15 \bar{y}^{2}+35 \bar{x}+25 \bar{y}-14$, we have $\operatorname{ord}_{\overline{\mathcal{F}}}\left(\bar{y}^{4}\right)=3$. Altogether, we see that $B$ is not a degree-filtered basis, since its order tuple is $(0,1,1,2,2,3,3,3)$.

In addition to these properties, recall that $\bar{t}_{1}=1$ in each degree filtered $K$-basis of $R_{\mathbb{X}}$ by Assumption 2.2 When $\mathcal{O}$ is a degree filtered $K$-basis of $R_{\mathbb{X}}$, the Hilbert function of $\mathcal{O}$ agrees with the Castelnuovo function of $\mathbb{X}$. For a discussion of these notions, we refer to [15], Section 5.

Using this terminology, the degree filtered border basis scheme is a subscheme of $\mathbb{B}_{\mathcal{O}}$ which parametrizes all 0-dimensional ideals in $P$ which have a degree filtered 
$\mathcal{O}$-border basis. The following proposition provides an explicit description of this subscheme.

Proposition 5.3. Let $\mathcal{O}=\left\{t_{1}, \ldots, t_{\mu}\right\}$ be an order ideal, let $\partial \mathcal{O}=\left\{b_{1}, \ldots, b_{\nu}\right\}$ be the border of $\mathcal{O}$, and let $G=\left\{g_{1}, \ldots, g_{\nu}\right\}$ be the generic $\mathcal{O}$-border prebasis, where $g_{j}=b_{j}-\sum_{i=1}^{\mu} c_{i j} t_{i}$.

(a) For a $K$-rational point $\Gamma=\left(\gamma_{i j}\right)$ of $\mathbb{B}_{\mathcal{O}}$, the 0-dimensional scheme $\mathbb{X}_{\Gamma}$ represented by $\Gamma$ has a degree filtered $\mathcal{O}$-border basis if and only if $\gamma_{i j}=0$ for all $i \in\{1, \ldots, \mu\}$ and $j \in\{1, \ldots, \nu\}$ such that $\operatorname{deg}\left(t_{i}\right)>\operatorname{deg}\left(b_{j}\right)$.

(b) Let $I_{\mathcal{O}}^{\mathrm{df}}$ be the ideal in $K[C]$ generated by all indeterminates $c_{i j}$ such that $\operatorname{deg}\left(t_{i}\right)>\operatorname{deg}\left(b_{j}\right)$. The $K$-rational points of the closed subscheme $\mathbb{B}_{\mathcal{O}}^{\mathrm{df}}$ of $\mathbb{B}_{\mathcal{O}}$ defined by the ideal $I\left(\mathbb{B}_{\mathcal{O}}\right)+I_{\mathcal{O}}^{\mathrm{df}}$ represent the 0-dimensional schemes $\mathbb{X}$ in $\mathbb{A}_{K}^{n}$ which have a degree filtered $\mathcal{O}$-border basis.

Proof. Claim (a) follows immediately from Condition (d) in [15, Prop. 5.3, and (b) is a consequence of (a).

As pointed out by the referee, this proposition shows that $\mathbb{B}_{\mathcal{O}}^{\text {df }}$ is the positive Białynicki-Birula decomposition of the scheme $\mathbb{B}_{\mathcal{O}}$. This follows from the general theory of Białynicki-Birula decompositions for affine schemes, see for example [7, Subsection 1.3.4 and [8], Prop. 4.5. Thus the scheme $\mathbb{B}_{\mathcal{O}}^{\text {df }}$ is linked to the geometry of the Białynicki-Birula decomposition for the Hilbert scheme, as $\mathbb{B}_{\mathcal{O}}^{\text {df }}$ is an open subscheme of it (see [7, Lemma 1.4.7 and [8], Prop. 5.2).

Part (b) of the preceding proposition gives rise to the following definition.

Definition 5.4. Let $\mathcal{O}=\left\{t_{1}, \ldots, t_{\mu}\right\}$ be an order ideal in $\mathbb{T}^{n}$, let $\partial \mathcal{O}=$ $\left\{b_{1}, \ldots, b_{\nu}\right\}$ be the border of $\mathcal{O}$, and let $I_{\mathcal{O}}^{\mathrm{df}}$ be the ideal in $K[C]$ generated by all indeterminates $c_{i j}$ such that $\operatorname{deg}\left(t_{i}\right)>\operatorname{deg}\left(b_{j}\right)$.

(a) The closed subscheme $\mathbb{B}_{\mathcal{O}}^{\text {df }}$ of $\mathbb{B}_{\mathcal{O}}$ defined by $I\left(\mathbb{B}_{\mathcal{O}}^{\mathrm{df}}\right)=I\left(\mathbb{B}_{\mathcal{O}}\right)+I_{\mathcal{O}}^{\text {df }}$ is called the degree filtered $\mathcal{O}$-border basis scheme. Its affine coordinate ring is denoted by $B_{\mathcal{O}}^{\mathrm{df}}=K[C] / I\left(\mathbb{B}_{\mathcal{O}}^{\mathrm{df}}\right)$.

(b) The set of polynomials $G^{\mathrm{df}}=\left\{g_{1}^{\mathrm{df}}, \ldots, g_{\nu}^{\mathrm{df}}\right\}$ in $K[C]\left[x_{1}, \ldots, x_{n}\right]$ given by $g_{j}=$ $b_{j}-\sum_{\left\{i \mid \operatorname{deg}\left(t_{i}\right) \leq \operatorname{deg}\left(b_{j}\right)\right\}} c_{i j} t_{i}$ for $j=1, \ldots, \nu$ is called the generic degree filtered $\mathcal{O}$-border prebasis.

(c) The canonical $B_{\mathcal{O}}^{\mathrm{df}}$-algebra homomorphism

$$
\Phi^{\mathrm{df}}: B_{\mathcal{O}}^{\mathrm{df}} \longrightarrow U_{\mathcal{O}}^{\mathrm{df}}:=B_{\mathcal{O}}^{\mathrm{df}}\left[x_{1}, \ldots, x_{n}\right] /\left\langle G^{\mathrm{df}}\right\rangle
$$

is called the universal degree filtered $\mathcal{O}$-border basis family.

Since the universal $\mathcal{O}$-border basis family is a free $B_{\mathcal{O}}$-module with basis $\mathcal{O}$ (cf. 20, Thm. 3.4), it follows by a base change that the universal degree filtered $\mathcal{O}$-border basis family is free with basis $\mathcal{O}$, too. The matrices defining the multiplication maps on $U_{\mathcal{O}}^{\text {df }}$ can be obtained as follows.

Remark 5.5. For $k=1, \ldots, n$, let $\mathcal{A}_{k} \in \operatorname{Mat}_{\mu}(K[C])$ be the $k$-th generic multiplication matrix with respect to $\mathcal{O}$ (see Definition 3.1, b), and let $C^{\text {nondf }}$ be the set of 
all indeterminates $c_{i j}$ such that $\operatorname{deg}\left(t_{i}\right)>\operatorname{deg}\left(b_{j}\right)$, i.e., the set of all indeterminates $c_{i j}$ of negative total arrow degree.

(a) For $k=1, \ldots, n$, let $\mathcal{A}_{k}^{\mathrm{df}}$ be the matrix obtained from $\mathcal{A}_{k}$ by setting all indeterminates in $C^{\text {nondf }}$ equal to zero. Then the matrix $\mathcal{A}_{k}^{\text {df }}$ describes the multiplication by $x_{k}$ on $U_{\mathcal{O}}^{\mathrm{df}}$ with respect to the basis $\mathcal{O}$. The matrices $\mathcal{A}_{1}^{\mathrm{df}}, \ldots, \mathcal{A}_{n}^{\mathrm{df}}$ are called the generic degree filtered multiplication matrices with respect to $\mathcal{O}$.

(b) For any polynomial $f \in P$, the multiplication by $f$ on $U_{\mathcal{O}}^{\mathrm{df}}$ is given by the matrix $f\left(\mathcal{A}_{1}^{\mathrm{df}}, \ldots, \mathcal{A}_{n}^{\mathrm{df}}\right)$ with respect to the basis $\mathcal{O}$.

Another useful observation is that the total arrow degree yields a non-negative grading of $B_{\mathcal{O}}^{\mathrm{df}}$ in the following sense.

Remark 5.6. Given the set $C=\left\{c_{i j} \mid i \in\{1, \ldots, \mu\}, j \in\{1, \ldots, \nu\}\right\}$, we let $C^{\mathrm{df}}=\left\{c_{i j} \in C \mid \operatorname{deg}\left(t_{i}\right) \leq \operatorname{deg}\left(b_{j}\right)\right\}$ and $C^{\text {nondf }}=C \backslash C^{\mathrm{df}}$. When we set the indeterminates in $C^{\text {nondf }}$ equal to zero in $I\left(\mathbb{B}_{\mathcal{O}}\right)$, we get an ideal $\bar{I}\left(\mathbb{B}_{\mathcal{O}}^{\text {df }}\right)$ such that

$$
B_{\mathcal{O}}^{\mathrm{df}}=K[C] / I\left(\mathbb{B}_{\mathcal{O}}^{\mathrm{df}}\right) \cong K\left[C^{\mathrm{df}}\right] / \bar{I}\left(\mathbb{B}_{\mathcal{O}}^{\mathrm{df}}\right)
$$

Notice that the indeterminates in $C^{\mathrm{df}}$ are precisely the elements of non-negative total arrow degree in $C$. Hence the isomorphism $(*)$ shows that the total arrow degree provides a non-negative grading of $B_{\mathcal{O}}^{\mathrm{df}}$. Another way of phrasing this observation is that the entries of the commutators of the matrices $\mathcal{A}_{i}^{\mathrm{df}}$ are homogeneous polynomials of non-negative degrees with respect to the total arrow degree.

The next proposition provides an important family of examples of degree filtered border bases. To prove it, we use the following auxiliary result.

Lemma 5.7. Let $\mathcal{O}=\left\{t_{1}, \ldots, t_{\mu}\right\}$ be an order ideal with border $\partial \mathcal{O}=\left\{b_{1}, \ldots, b_{\nu}\right\}$. Recall that $\operatorname{deg}\left(t_{1}\right) \leq \cdots \leq \operatorname{deg}\left(t_{\mu}\right)$, and w.l.o.g. let $\operatorname{deg}\left(b_{1}\right) \leq \cdots \leq \operatorname{deg}\left(b_{\nu}\right)$.

Assume that $\operatorname{deg}\left(t_{\mu}\right)>\operatorname{deg}\left(b_{1}\right)$, and let $\Gamma=\left(\gamma_{i j}\right) \in K^{\mu \nu}$ with $\gamma_{\mu 1}=1$ and $\gamma_{i j}=0$ for $(i, j) \neq(\mu, 1)$. Then $\Gamma$ represents a 0 -dimensional scheme $\mathbb{X}_{\Gamma}$ in $\mathbb{B}_{\mathcal{O}}$.

Proof. To show that the $\mathcal{O}$-border prebasis $G_{\Gamma}=\left(g_{1}, \ldots, g_{\nu}\right)=\left(b_{1}-t_{\mu}, b_{2}, \ldots, b_{\nu}\right)$ is an $\mathcal{O}$-border basis, it suffices to show that the residue classes of the elements of $\mathcal{O}$ form a $K$-basis of $P / I_{\Gamma}$, where $I_{\Gamma}=\left\langle G_{\Gamma}\right\rangle$.

Since $\mathcal{O}$ is an order ideal, we have $b_{1} \nmid t_{\mu}$. Therefore there exists a term ordering $\sigma$ such that $b_{1}>_{\sigma} t_{\mu}$. Now we use Buchberger's Criterion to verify that $G_{\Gamma}=\left(b_{1}-t_{\mu}, b_{2}, \ldots, b_{\nu}\right)$ is a $\sigma$-Gröbner basis of $I_{\Gamma}$.

For this it suffices to show that, for $k=2, \ldots, \nu$, the $S$-polynomial $S_{1 k}$ satisfies $S_{1 k} \stackrel{G}{\rightarrow} 0$. Using

$$
S_{1 k}=\frac{\operatorname{lcm}\left(b_{1}, b_{k}\right)}{b_{1}}\left(b_{1}-t_{\mu}\right)-\frac{\operatorname{lcm}\left(b_{1}, b_{k}\right)}{b_{k}} b_{k}=-\frac{\operatorname{lcm}\left(b_{1}, b_{k}\right)}{b_{1}} t_{\mu}
$$

and the fact that $\operatorname{deg}\left(b_{1}\right) \leq \operatorname{deg}\left(b_{k}\right)$ implies $b_{k} \nmid b_{1}$, we see that $S_{1 k}$ is a proper multiple of $t_{\mu}$. Therefore the claim follows from $x_{i} t_{\mu} \in \partial \mathcal{O} \backslash\left\{b_{1}\right\} \subset G_{\Gamma}$. Now 
we conclude from Macaulay's Basis Theorem that $\mathcal{O}=\mathcal{O}_{\sigma}\left(I_{\Gamma}\right)=\mathbb{T}^{n} \backslash \operatorname{LT}_{\sigma}\left(G_{\Gamma}\right)$ represents a $K$-basis of $P / I_{\Gamma}$, and the proof is complete.

At this point we are ready for the following result.

Proposition 5.8. For an order ideal $\mathcal{O}=\left\{t_{1}, \ldots, t_{\mu}\right\}$, the following conditions are equivalent.

(a) We have $\mathbb{B}_{\mathcal{O}}=\mathbb{B}_{\mathcal{O}}^{\mathrm{df}}$.

(b) For $i=1, \ldots, \mu$ and $j=1, \ldots, \nu$, we have $\operatorname{deg}\left(t_{i}\right) \leq \operatorname{deg}\left(b_{j}\right)$.

(c) The order ideal $\mathcal{O}$ has the generic Hilbert function, i.e., for $i \geq 0$ we have $\#\left\{t_{j} \in \mathcal{O} \mid \operatorname{deg}\left(t_{j}\right) \leq i\right\}=\min \left\{\mu, \# \mathbb{T}_{\leq i}^{n}\right\}$.

If these conditions are satisfied, we say that $\mathcal{O}$ has a maxdeg border.

Proof. First we show that (a) implies (b). By hypothesis, every scheme $\mathbb{X}_{\Gamma}$ represented by a $K$-rational point $\Gamma$ of $\mathbb{B}_{\mathcal{O}}$ has an $\mathcal{O}$-border bases which is degree filtered. Suppose that there exist terms $t_{i} \in \mathcal{O}$ and $b_{j} \in \partial \mathcal{O}$ such that $\operatorname{deg}\left(t_{i}\right)>\operatorname{deg}\left(b_{j}\right)$. Then the lemma yields a 0 -dimensional scheme $\mathbb{X}_{\Gamma}$ which has an $\mathcal{O}$-border basis that is not degree filtered, in contradiction to the hypothesis.

Since $(b) \Leftrightarrow(c)$ is clear, it suffices to note that (b) $\Rightarrow$ (a) follows from [15], Prop. 5.3 in order to finish the proof.

Degree filtered border bases also arise naturally from degree compatible term orderings as follows.

Example 5.9. Let $\mathbb{X}$ be a 0 -dimensional scheme in $\mathbb{A}_{K}^{n}$, and let $\sigma$ be a degree compatible term ordering. Then $\mathbb{X}$ has a degree filtered border basis with respect to $\mathcal{O}_{\sigma}\left(I_{\mathbb{X}}\right)=\mathbb{T}^{n} \backslash \operatorname{LT}_{\sigma}\left(I_{\mathbb{X}}\right)$. This follows from the observation that a border basis element $g_{j}=b_{j}-\sum_{i=1}^{\mu} \gamma_{i j} t_{i}$ can be interpreted as saying that the normal form of $b_{j}$ with respect to a $\sigma$-Gröbner basis of $I_{\mathbb{X}}$ is $\sum_{i=1}^{\mu} \gamma_{i j} t_{i}$, and since $\sigma$ is degree compatible, this implies $\operatorname{deg}\left(\sum_{i=1}^{\mu} \gamma_{i j} t_{i}\right) \leq \operatorname{deg}\left(b_{j}\right)$. Hence we have $b_{j} \in \operatorname{DF}\left(g_{j}\right)$, Condition (d) of [15], Prop. 5.3 is satisfied, and the border basis is degree filtered.

A particular case of a degree filtered border basis is a homogeneous one. The family of all homogeneous border bases can be parametrized as follows (see 20, Section 5).

Definition 5.10. Let $\mathcal{O}=\left\{t_{1}, \ldots, t_{\mu}\right\}$ be an order ideal, let $\partial \mathcal{O}=\left\{b_{1}, \ldots, b_{\nu}\right\}$ be the border of $\mathcal{O}$, and let $I_{\mathcal{O}}^{\text {hom }}$ be the ideal in $K[C]$ generated by all indeterminates $c_{i j}$ such that $\operatorname{deg}\left(t_{i}\right) \neq \operatorname{deg}\left(b_{j}\right)$.

(a) The closed subscheme $\mathbb{B}_{\mathcal{O}}^{\text {hom }}$ of $\mathbb{B}_{\mathcal{O}}$ defined by $I\left(\mathbb{B}_{\mathcal{O}}^{\text {hom }}\right)=I\left(\mathbb{B}_{\mathcal{O}}\right)+I_{\mathcal{O}}^{\text {hom }}$ is called the homogeneous $\mathcal{O}$-border basis scheme. Its affine coordinate ring is denoted by $B_{\mathcal{O}}^{\text {hom }}=K[C] / I\left(\mathbb{B}_{\mathcal{O}}^{\text {hom }}\right)$.

(b) The set of polynomials $G^{\text {hom }}=\left\{g_{1}^{\text {hom }}, \ldots, g_{\nu}^{\text {hom }}\right\}$ in $K[C]\left[x_{1}, \ldots, x_{n}\right]$ given by $g_{j}=b_{j}-\sum_{\left\{i \mid \operatorname{deg}\left(t_{i}\right)=\operatorname{deg}\left(b_{j}\right)\right\}} c_{i j} t_{i}$ for $j=1, \ldots, \nu$ is called the generic homogeneous $\mathcal{O}$-border prebasis. 
(c) The canonical $B_{\mathcal{O}}^{\text {hom }}$-algebra homomorphism

$$
\Phi^{\text {hom }}: B_{\mathcal{O}}^{\text {hom }} \longrightarrow U_{\mathcal{O}}^{\text {hom }}:=B_{\mathcal{O}}^{\text {hom }}\left[x_{1}, \ldots, x_{n}\right] /\left\langle G^{\text {hom }}\right\rangle
$$

is called the universal homogeneous $\mathcal{O}$-border basis family.

Again it follows by a base change from [20, Thm. 3.4, that the universal homogeneous $\mathcal{O}$-border basis family is free with basis $\mathcal{O}$. The matrices defining the multiplication maps on $U_{\mathcal{O}}^{\text {hom }}$ can be described as follows.

Remark 5.11. For $k=1, \ldots, n$, let $\mathcal{A}_{k} \in \operatorname{Mat}_{\mu}(K[C])$ be the $k$-th generic multiplication matrix with respect to $\mathcal{O}$ (see Definition 3.1.b), and let $C^{\text {nonhom }}$ be the set of all indeterminates $c_{i j}$ such that $\operatorname{deg}\left(t_{i}\right) \neq \operatorname{deg}\left(b_{j}\right)$.

(a) For $k=1, \ldots, n$, let $\mathcal{A}_{k}^{\text {hom }}$ be the matrix obtained from $\mathcal{A}_{k}$ by setting all indeterminates in $C^{\text {nonhom }}$ equal to zero. Then the matrix $\mathcal{A}_{k}^{\text {hom }}$ represents the multiplication map by $x_{k}$ on $U_{\mathcal{O}}^{\text {hom }}$ with respect to the basis $\mathcal{O}$. The matrices $\mathcal{A}_{1}^{\text {hom }}, \ldots, \mathcal{A}_{n}^{\text {hom }}$ are called the generic homogeneous multiplication matrices with respect to $\mathcal{O}$.

(b) For any polynomial $f \in P$, the multiplication by $f$ on $U_{\mathcal{O}}^{\text {hom }}$ is given by the matrix $f\left(\mathcal{A}_{1}^{\text {hom }}, \ldots, \mathcal{A}_{n}^{\text {hom }}\right)$ with respect to the basis $\mathcal{O}$.

It is clear that the homogeneous $\mathcal{O}$-border basis scheme $\mathbb{B}_{\mathcal{O}}^{\text {hom }}$ is a closed subscheme of $\mathbb{B}_{\mathcal{O}}^{\text {df }}$. Let us also look at the total arrow degree on $B_{\mathcal{O}}^{\text {hom }}$.

Remark 5.12. Let $C=\left\{c_{i j} \mid i \in\{1, \ldots, \mu\}, j \in\{1, \ldots, \nu\}\right\}$, let $C^{\mathrm{df}}=\left\{c_{i j} \in\right.$ $\left.C \mid \operatorname{deg}\left(t_{i}\right) \leq \operatorname{deg}\left(b_{j}\right)\right\}$, and let $C^{\text {hom }}=\left\{c_{i j} \in C \mid \operatorname{deg}\left(t_{i}\right)=\operatorname{deg}\left(b_{j}\right)\right\}$. Then the indeterminates in $C^{\text {hom }}$ are precisely the elements of total arrow degree zero in $C$. Hence the entries of the commutators of the matrices $\mathcal{A}_{i}^{\text {hom }}$ are homogeneous polynomials of degree zero with respect to the total arrow degree. Since they generate $I\left(\mathbb{B}_{\mathcal{O}}^{\text {hom }}\right)$, we obtain an isomorphism of graded $K$-algebras

$$
B_{\mathcal{O}}^{\text {hom }}=K[C] / I\left(\mathbb{B}_{\mathcal{O}}^{\text {hom }}\right) \cong K\left[C^{\text {hom }}\right] / \bar{I}\left(\mathbb{B}_{\mathcal{O}}^{\text {hom }}\right)
$$

where $\bar{I}\left(\mathbb{B}_{\mathcal{O}}^{\text {hom }}\right)$ is the result of setting all indeterminates in $C^{\text {nonhom }}=C \backslash C^{\text {hom }}$ equal to zero in $I\left(\mathbb{B}_{\mathcal{O}}\right)$ (or in $I\left(\mathbb{B}_{\mathcal{O}}^{\text {df }}\right)$ ), and where the total arrow degree induces the trivial grading on $B_{\mathcal{O}}^{\text {hom }}$.

By combining this remark with Remark 5.6, we get the following useful result.

Proposition 5.13. Given the set $C=\left\{c_{i j} \mid i \in\{1, \ldots, \mu\}, j \in\{1, \ldots, \nu\}\right\}$, we let $C^{\mathrm{df}}=\left\{c_{i j} \in C \mid \operatorname{deg}\left(t_{i}\right) \leq \operatorname{deg}\left(b_{j}\right)\right\}$ and $C^{\mathrm{hom}}=\left\{c_{i j} \in C \mid \operatorname{deg}\left(t_{i}\right)=\operatorname{deg}\left(b_{j}\right)\right\}$.

(a) The defining ideal $\bar{I}\left(\mathbb{B}_{\mathcal{O}}^{\text {hom }}\right)$ of $\mathbb{B}_{\mathcal{O}}^{\text {hom }}$ in $K\left[C^{\text {hom }}\right]$ is exactly the set of homogeneous elements of degree zero of the defining ideal $\bar{I}\left(\mathbb{B}_{\mathcal{O}}^{\mathrm{df}}\right)$ in $K\left[C^{\mathrm{df}}\right]$ with respect to the total arrow degree. In particular, we have $\bar{I}\left(\mathbb{B}_{\mathcal{O}}^{\text {hom }}\right)=\bar{I}\left(\mathbb{B}_{\mathcal{O}}^{\text {df }}\right) \cap K\left[C^{\text {hom }}\right]$ and $\bar{I}\left(\mathbb{B}_{\mathcal{O}}^{\text {hom }}\right) \cdot K\left[C^{\mathrm{df}}\right] \subseteq \bar{I}\left(\mathbb{B}_{\mathcal{O}}^{\mathrm{df}}\right)$.

(b) We have a canonical injective $K$-algebra homomorphism $B_{\mathcal{O}}^{\text {hom }} \hookrightarrow B_{\mathcal{O}}^{\mathrm{df}}$, and the elements of $B_{\mathcal{O}}^{\text {hom }}$ are precisely the elements of total arrow degree zero in $B_{\mathcal{O}}^{\mathrm{df}}$. 
In more geometric jargon, this proposition shows that $\mathbb{B}_{\mathcal{O}}^{\text {hom }}$ is the $\mathbb{G}_{m}$-fixed locus of $\mathbb{B}_{\mathcal{O}}$, and hence an open affine subscheme of the $\mathbb{G}_{m}$-fixed locus of the Hilbert scheme. Thus the closed immersion $\mathbb{B}_{\mathcal{O}}^{\text {hom }} \hookrightarrow \mathbb{B}_{\mathcal{O}}^{\text {df }}$ can be interpreted geometrically as the retraction of the Białynicki-Birula decomposition to the $\mathbb{G}_{m}$-fixed locus.

To finish the section, let us compute the subschemes $\mathbb{B}_{\mathcal{O}}^{\text {df }}$ and $\mathbb{B}_{\mathcal{O}}^{\text {hom }}$ of $\mathbb{B}_{\mathcal{O}}$ in the setting of Example 3.6.

Example 5.14. For the order ideal $\mathcal{O}=\{1, x, y, x y\}$ in $\mathbb{T}^{2}$, we have calculated the ideal $I\left(\mathbb{B}_{\mathcal{O}}\right)$ in Example 3.6 Let us also determine $I\left(\mathbb{B}_{\mathcal{O}}^{\text {df }}\right)$ and $I\left(\mathbb{B}_{\mathcal{O}}^{\text {hom }}\right)$ in this case.

(a) To get the defining ideal of $\mathbb{B}_{\mathcal{O}}^{\text {df }}$, we find $C^{\text {nondf }}=\left\{c_{i j} \mid \operatorname{deg}\left(b_{j}\right)<\operatorname{deg}\left(t_{i}\right)\right\}$ first. In the current example we get $C^{\text {nondf }}=\emptyset$, and therefore $I\left(\mathbb{B}_{\mathcal{O}}^{\text {df }}\right)=I\left(\mathbb{B}_{\mathcal{O}}\right)$. In other words, we have $\mathbb{B}_{\mathcal{O}}^{\mathrm{df}}=\mathbb{B}_{\mathcal{O}}$.

(b) The defining ideal of $\mathbb{B}_{\mathcal{O}}^{\text {hom }}$ is obtained by adding the ideal generated by

$$
\begin{aligned}
C^{\text {nonhom }} & =\left\{c_{i j} \mid \operatorname{deg}\left(b_{j}\right) \neq \operatorname{deg}\left(t_{i}\right)\right\} \\
& =\left\{c_{11}, c_{12}, c_{13}, c_{14}, c_{21}, c_{22}, c_{23}, c_{24}, c_{31}, c_{32}, c_{33}, c_{34}, c_{43}, c_{44}\right\}
\end{aligned}
$$

to $I\left(\mathbb{B}_{\mathcal{O}}\right)$, and after simplifying the generators of $I\left(\mathbb{B}_{\mathcal{O}}\right)$, we obtain $I\left(\mathbb{B}_{\mathcal{O}}^{\text {hom }}\right)=\left\langle C^{\text {nonhom }}\right\rangle$. In other words, the scheme $\mathbb{B}_{\mathcal{O}}^{\text {hom }}$ is equal to the plane $\operatorname{Spec}\left(K\left[c_{41}, c_{42}\right]\right)$.

\section{The Cayley-Bacharach Locus in $\mathbb{B}_{\mathcal{O}}^{\mathrm{df}}$}

In the following we want to describe the locus of all $K$-rational points of the border basis scheme which represent 0-dimensional affine schemes $\mathbb{X}$ having the CayleyBacharach property. Since the description of this property requires us to fix the Hilbert function of $\mathbb{X}$, we have to work in an appropriate subscheme of $\mathbb{B}_{\mathcal{O}}$. When the Hilbert function of $\mathbb{X}$ corresponds to the degrees of the terms in $\mathcal{O}$, the appropriate subscheme of $\mathbb{B}_{\mathcal{O}}$ is the degree filtered border basis scheme. Hence we look for a description of the Cayley-Bacharach locus in $\mathbb{B}_{\mathcal{O}}^{\text {df }}$. Let us start by recalling the definition of the Cayley-Bacharach property in the general setting (see [14, Definition 3.10).

Let $\mathbb{X}$ be a 0 -dimensional subscheme of $\mathbb{A}_{K}^{n}$, let $I_{\mathbb{X}} \subseteq P$ be the vanishing ideal of $\mathbb{X}$, and let $R_{\mathbb{X}}=P / I_{\mathbb{X}}$ be the coordinate ring of $\mathbb{X}$. Then the primary decomposition of the vanishing ideal $I_{\mathbb{X}}$ of $\mathbb{X}$ has the form

$$
I_{\mathbb{X}}=\mathfrak{Q}_{1} \cap \cdots \cap \mathfrak{Q}_{s}
$$

where $\mathfrak{Q}_{i} \subset P$ is an $\mathfrak{M}_{i}$-primary ideal with a maximal ideal $\mathfrak{M}_{i} \subset P$ for each $i \in\{1, \ldots, s\}$. The image of the maximal ideal $\mathfrak{M}_{i}$ in $R_{\mathbb{X}}$ is denoted by $\mathfrak{m}_{i}$ for $i \in\{1, \ldots, s\}$. Clearly, the ideals $\mathfrak{m}_{1}, \ldots, \mathfrak{m}_{s}$ are the maximal ideals of $R_{\mathbb{X}}$. In this setting, the following definitions were introduced in [14].

Definition 6.1. Let $\mathbb{X}$ be a 0 -dimensional subscheme of $\mathbb{A}_{K}^{n}$ as above. 
(a) For $i \in\{1, \ldots, s\}$, an ideal $J \subset P$ is called a minimal $\mathfrak{Q}_{i}$-divisor of $I_{\mathbb{X}}$ if $J=\mathfrak{Q}_{1} \cap \cdots \cap \mathfrak{Q}_{i}^{\prime} \cap \cdots \cap \mathfrak{Q}_{s}$ with an ideal $\mathfrak{Q}_{i}^{\prime} \subset P$ such that $\mathfrak{Q}_{i} \subset \mathfrak{Q}_{i}^{\prime} \subseteq \mathfrak{M}_{i}$ and $\operatorname{dim}_{K}\left(\mathfrak{Q}_{i}^{\prime} / \mathfrak{Q}_{i}\right)=\operatorname{dim}_{K}\left(P / \mathfrak{M}_{i}\right)$.

(b) For $i \in\{1, \ldots, s\}$ and a minimal $\mathfrak{Q}_{i}$-divisor $J$ of $I_{\mathbb{X}}$, we let $\operatorname{ri}\left(J / I_{\mathbb{X}}\right)=$ $\max \left\{\operatorname{ord}_{\mathcal{F}}(f) \mid f \in J / I_{\mathbb{X}} \backslash\{0\}\right\}$. Then the number

$$
\operatorname{sepdeg}\left(\mathfrak{m}_{i}\right)=\min \left\{\operatorname{ri}\left(J / I_{\mathbb{X}}\right) \mid J \text { is a minimal } \mathfrak{Q}_{i} \text {-divisor of } I_{\mathbb{X}}\right\}
$$

is called the separator degree of $\mathfrak{m}_{i}$ in $R_{\mathbb{X}}$.

(c) The scheme $\mathbb{X}$ is called a Cayley-Bacharach scheme, or the ring $R_{\mathbb{X}}$ is said to have the Cayley-Bacharach property, if we have $\operatorname{sepdeg}\left(\mathfrak{m}_{i}\right)=\operatorname{ri}\left(R_{\mathbb{X}}\right)$ for $i=1, \ldots, s$.

In [14, Section 3, it is shown that this definition generalizes the classical definition of the Cayley-Bacharach property for a set of points $\mathbb{X}$ in an affine or projective space over an algebraically closed field. Recall that this classical definition is usually phrased by requiring that every hypersurface of degree $\operatorname{ri}\left(R_{\mathbb{X}}\right)-1$ which contains all points of $\mathbb{X}$ but one automatically contains the last point.

Furthermore, in [14, Alg. 4.6, we provided an algorithm which checks the Cayley-Bacharach property of $R_{\mathbb{X}}$ using the multiplication matrices of the canonical module of $R_{\mathbb{X}}$. This result will be the basis of our algorithm below. First we introduce the following terminology.

Definition 6.2. Let $\mathcal{O}=\left\{t_{1}, \ldots, t_{\mu}\right\}$ be an order ideal in $\mathbb{T}^{n}$. Then the set of all $K$-rational points $\Gamma=\left(\gamma_{i j}\right) \in K^{\mu \nu}$ of the border basis scheme $\mathbb{B}_{\mathcal{O}}$ which represent a 0-dimensional Cayley-Bacharach scheme $\mathbb{X}_{\Gamma}$ is called the set of CayleyBacharach points of $\mathbb{B}_{\mathcal{O}}$.

As the following algorithms shows, there exists an open subscheme $\mathrm{CB}_{\mathcal{O}}^{\mathrm{df}}$ of $\mathbb{B}_{\mathcal{O}}^{\mathrm{df}}$ whose $K$-rational points are precisely the set of Cayley-Bacharach points of $\mathbb{B}_{\mathcal{O}}^{\mathrm{df}}$. It is called the Cayley-Bacharach locus in $\mathbb{B}_{\mathcal{O}}^{\text {df }}$. More precisely, we compute the equations of a closed subscheme of $\mathbb{B}_{\mathcal{O}}^{\mathrm{df}}$ which forms the complement of $\mathrm{CB}_{\mathcal{O}}^{\mathrm{df}}$.

\footnotetext{
Algorithm 6.3. (Computing the Cayley-Bacharach Locus in $\mathbb{B}_{\mathcal{O}}^{\text {df }}$ ) Let $\mathcal{O}=\left\{t_{1}, \ldots, t_{\mu}\right\}$ be an order ideal in $\mathbb{T}^{n}$, and let $\Delta=\#\{i \in\{1, \ldots, \mu\}$ | $\left.\operatorname{deg}\left(t_{i}\right)=\operatorname{deg}\left(t_{\mu}\right)\right\}$. Consider the following sequence of instructions.

(1) Using Proposition 5.3. b, calculate $I\left(\mathbb{B}_{\mathcal{O}}^{\mathrm{df}}\right)=I\left(\mathbb{B}_{\mathcal{O}}\right)+I_{\mathcal{O}}^{\mathrm{df}}$.

(2) Construct the generic degree filtered multiplication matrices $\mathcal{A}_{1}^{\mathrm{df}}, \ldots, \mathcal{A}_{n}^{\mathrm{df}}$. For $i=1, \ldots, \mu$, compute the multiplication matrix $M_{t_{i}}=t_{i}\left(\mathcal{A}_{1}^{\mathrm{df}}, \ldots, \mathcal{A}_{n}^{\mathrm{df}}\right)$.

(3) For $j=1, \ldots, \Delta$, form the matrix $V_{j} \in \operatorname{Mat}_{\mu}(K[C])$ whose $i$-th column is the $(\mu-\Delta+j)$-th column of $M_{t_{i}}^{\text {tr }}$ for $i=1, \ldots, \mu$.

(4) Form the block column matrix $W=\operatorname{Col}\left(V_{1}, \ldots, V_{\Delta}\right)$ and compute the ideal $J_{\mathcal{O}}$ in $K[C]$ generated by the maximal minors of $W$.

(5) Return the ideal $I\left(\mathbb{B}_{\mathcal{O}}^{\mathrm{df}}\right)+J_{\mathcal{O}}$.
} 
This is an algorithm which computes an ideal in $K[C]$. This ideal defines a closed subscheme $\mathrm{NonCB}_{\mathcal{O}}^{\mathrm{df}}$ whose $K$-rational points represent those 0-dimensional subschemes of $\mathbb{A}_{K}^{n}$ which have a degree filtered $\mathcal{O}$-border basis, but are not CayleyBacharach schemes.

Proof. A $K$-rational rational point $\Gamma=\left(\gamma_{i j}\right)$ of $\mathbb{B}_{\mathcal{O}}^{\text {df }}$ corresponds to a zero of the ideal $I\left(\mathbb{B}_{\mathcal{O}}^{\mathrm{df}}\right)$ which is computed in Step (1). According to Algorithm 4.6 of [14, the 0-dimensional scheme $\mathbb{X}_{\Gamma}$ represented by $\Gamma$ is a Cayley-Bacharach scheme if and only if $\Gamma$ is a zero of the ideal generated by the maximal minors of $W$. This observation finishes the proof.

In view of this algorithm, the Cayley-Bacharach locus in $\mathbb{B}_{\mathcal{O}}^{\text {df }}$ is the open subscheme $\mathrm{CB}_{\mathcal{O}}^{\mathrm{df}}=\mathbb{B}_{\mathcal{O}}^{\mathrm{df}} \backslash \mathrm{NonCB}_{\mathcal{O}}^{\mathrm{df}}$ of the degree filtered $\mathcal{O}$-border basis scheme. Let us compute it for the following order ideal.

Example 6.4. Let $K$ be a field, and let $\mathcal{O}$ be the order ideal $\mathcal{O}=\left\{1, x, y, z, x^{2}\right\}$ in $P=K[x, y, z]$. Then we have $\partial \mathcal{O}=\left\{x y, y^{2}, x z, y z, z^{2}, x^{3}, x^{2} y, x^{2} z\right\}$ and thus $\mu=5$ as well as $\nu=8$. The ring $K[C]$ has 40 indeterminates, and the ideal $I\left(\mathbb{B}_{\mathcal{O}}\right)$ has 60 generators. Since $\mathcal{O}$ has the generic Hilbert function, we have $\mathbb{B}_{\mathcal{O}}=\mathbb{B}_{\mathcal{O}}^{\text {df }}$ by Proposition 5.8

When we calculate an ideal defining $\mathrm{NonCB}_{\mathcal{O}}^{\mathrm{df}}$ using the algorithm, we have $\Delta=1$ and

$$
W=V_{1}=\left(\begin{array}{ccccc}
0 & 0 & 0 & 0 & 1 \\
0 & 1 & c_{51} & c_{53} & c_{56} \\
0 & c_{51} & c_{52} & c_{54} & c_{31} c_{51}+c_{41} c_{53}+c_{51} c_{56}+c_{21} \\
0 & c_{53} & c_{54} & c_{55} & c_{33} c_{51}+c_{43} c_{53}+c_{53} c_{56}+c_{23} \\
1 & c_{56} & c_{57} & c_{58} & c_{36} c_{51}+c_{46} c_{53}+c_{56}^{2}+c_{26}
\end{array}\right)
$$

Consequently, the algorithm returns the ideal $I\left(\mathbb{B}_{\mathcal{O}}^{\mathrm{df}}\right)+\langle\operatorname{det}(W)\rangle=I\left(\mathbb{B}_{\mathcal{O}}^{\mathrm{df}}\right)+\langle f\rangle$, where $f=c_{52} c_{53}^{2}-2 c_{51} c_{53} c_{54}+c_{51}^{2} c_{55}+c_{54}^{2}-c_{52} c_{55}$. Thus the Cayley-Bacharach locus in $\mathbb{B}_{\mathcal{O}}^{\mathrm{df}}$ is the complement of the hypersurface section of $\mathbb{B}_{\mathcal{O}}^{\mathrm{df}}$ given by $f=0$.

\section{The Strict Cayley-Bacharach and Strict Gorenstein Loci in $\mathbb{B}_{\mathcal{O}}^{\text {df }}$}

Stronger properties than the Cayley-Bacharach property are defined as follows.

Definition 7.1. Let $\mathbb{X}$ be a 0 -dimensional scheme in $\mathbb{A}_{K}^{n}$.

(a) The scheme $\mathbb{X}$ is called a strict Cayley-Bacharach scheme if the graded ring $\operatorname{gr}_{\mathcal{F}}\left(R_{\mathbb{X}}\right)$ has the Cayley-Bacharach property.

(b) The scheme $\mathbb{X}$ is called a strict Gorenstein scheme if the graded ring $\operatorname{gr}_{\mathcal{F}}\left(R_{\mathbb{X}}\right)$ is a Gorenstein ring.

In [14, Section 6, we showed that every strict Cayley-Bacharach scheme is a Cayley-Bacharach scheme. 


\subsection{Checking the Strict Cayley-Bacharach Property}

In order to check schemes for the strict Cayley-Bacharach property, we may use the following algorithm which follows from [14, Alg. 4.6.

\section{Algorithm 7.2. (Checking Strict Cayley-Bacharach Schemes)}

Let $\mathbb{X}$ be a 0-dimensional scheme in $\mathbb{A}_{K}^{n}$ with affine coordinate ring $R_{\mathbb{X}}=P / I_{\mathbb{X}}$, and let $\mu=\operatorname{dim}_{K}\left(R_{\mathbb{X}}\right)$. Consider the following sequence of instructions.

(1) Compute an order ideal $\mathcal{O}=\left\{t_{1}, \ldots, t_{\mu}\right\}$ in $\mathbb{T}^{n}$ such that $\mathcal{O}$ is a degree filtered $K$-basis of $R_{\mathbb{X}}$. Let $\Delta \geq 1$ be such that $t_{\mu-\Delta+1}, \ldots, t_{\mu}$ are the elements of $\mathcal{O}$ of order $\operatorname{ri}\left(R_{\mathbb{X}}\right)$.

(2) For $i=1, \ldots, \mu$, compute the matrix $M_{\bar{t}_{i}} \in \operatorname{Mat}_{\mu}(K)$ representing the multiplication by $\bar{t}_{i}$ on $\operatorname{gr}_{\mathcal{F}}\left(R_{\mathbb{X}}\right) \cong R_{\mathbb{X}} / \mathrm{DF}\left(I_{\mathbb{X}}\right)$ with respect to the $K$-basis $\overline{\mathcal{O}}=\left(\bar{t}_{1}, \ldots, \bar{t}_{\mu}\right)$.

(3) For $j=1, \ldots, \Delta$, form the matrix $V_{j} \in \operatorname{Mat}_{\mu}(K)$ whose $i$-th column is the $(\mu-\Delta+j)$-th column of $\left(M_{\bar{t}_{i}}\right)^{\text {tr }}$ for $i=1, \ldots, \mu$.

(4) Form the block column matrix $W=\operatorname{Col}\left(V_{1}, \ldots, V_{\Delta}\right)$ and compute the maximal minors of $W$.

(5) If one of these maximal minors is non-zero, return TRUE. Otherwise, return FALSE.

This is an algorithm which checks whether $\mathbb{X}$ is a strict Cayley-Bacharach scheme and returns the corresponding Boolean value.

For an example of the application of this algorithm, see [14, Ex. 6.11.

A strict Gorenstein scheme is always locally Gorenstein (see for instance [14, Thm. 6.8), but the converse is not true in general. Moreover, a strict Gorenstein scheme is always a strict Cayley-Bacharach scheme (see [14, Thm. 6.12). The Hilbert function of the graded $\operatorname{ring} \operatorname{gr}_{\mathcal{F}}\left(R_{\mathbb{X}}\right)$ is given by the Castelnuovo function of $\mathbb{X}$, i.e., by the first difference function of $\mathrm{HF}_{\mathbb{X}}^{a}$. The following property is essential here.

Definition 7.3. For an order ideal $\mathcal{O}=\left\{t_{1}, \ldots, t_{\mu}\right\} \subseteq \mathbb{T}^{n}$, let $\operatorname{HF}_{\mathcal{O}}=\left(h_{0}, h_{1}, \ldots\right)$ be the affine Hilbert function of $\mathcal{O}$, and let $\varrho=\max \left\{i \geq 0 \mid h_{i}>0\right\}$. We say that $\mathrm{HF}_{\mathcal{O}}$ is symmetric if $h_{i}=h_{\varrho-i}$ for $i=1, \ldots, \varrho$.

Notice that if $\mathcal{O}$ is a degree filtered $K$-basis of $R_{\mathbb{X}}$ then this definition agrees with the definition of the symmetry of the Hilbert function of $\mathbb{X}$ given in [14], Def. 6.4. It is known that the affine Hilbert function of a strict Gorenstein scheme is symmetric (see for instance [14, Thm. 6.8). More precisely, a 0-dimensional affine scheme $\mathbb{X}$ is a strict Gorenstein scheme if and only if $\mathrm{HF}_{\mathbb{X}}^{a}$ is symmetric and $\mathbb{X}$ is a strict Cayley-Bacharach scheme (see 14, Thms. 6.8 and 6.12). Thus, using Algorithm 7.2 , we can check whether $\mathbb{X}$ is strictly Gorenstein in the following way.

Corollary 7.4. (Checking Strict Gorenstein Schemes)

In the setting of Algorithm 7.2, the following instructions define an algorithm which 
checks whether $\mathbb{X}$ is a strict Gorenstein scheme and returns the corresponding Boolean value.

(1) Compute an order ideal $\mathcal{O}=\left\{t_{1}, \ldots, t_{\mu}\right\}$ which represents a degree filtered $K$-basis of $R_{\mathbb{X}}$.

(2) Check whether $\mathrm{HF}_{\mathcal{O}}$ is symmetric. If this is not the case, return FALSE and stop.

(3) Using Algorithm 7.2, check whether $\mathbb{X}$ is a strict Cayley-Bacharach scheme and return the corresponding Boolean value.

Remark 7.5. Notice that, in the last part of this algorithm, it would have been sufficient to check whether $\mathbb{X}$ is a Cayley-Bacharach scheme. However, the more stringent condition of Algorithm 7.2 yields, in general, a more efficient test. Moreover, notice that the last steps in Algorithm 7.2 simplify here, because we have $\Delta=1$ and therefore only one matrix $V_{1}$ and one maximal minor $\operatorname{det}\left(V_{1}\right)$.

Let us apply the preceding algorithm to some concrete cases.

Example 7.6. Let $K=\mathbb{Q}$, let $P=K[x, y, z]$, and let $\mathbb{X}$ be the 0 -dimensional subscheme of $\mathbb{A}_{K}^{n}$ defined by the ideal $I_{\mathbb{X}}=\left\langle y^{2}-x^{2}, z^{2}-x^{2}, x y, x z, y z, x^{3}\right\rangle$. Since the ideal $I_{\mathbb{X}}$ is homogeneous, we have $\operatorname{DF}\left(I_{\mathbb{X}}\right)=I_{\mathbb{X}}$. Moreover, from the fact that the given system of generators is the reduced Gröbner basis of $I_{\mathbb{X}}$ with respect to the DegRevLex term ordering such that $z>y>x$, it follows that a degree filtered $K$-basis of $R_{\mathbb{X}}$ is given by $\mathcal{O}=\left\{1, x, y, z, x^{2}\right\}$.

Thus the Hilbert function of $\mathcal{O}$ is $(1,3,1)$ which is symmetric. Hence Algorithm 7.4 asks us to compute the multiplication matrices for the elements of $\mathcal{O}$ and to combine their last columns. We get the matrix

$$
V=\left(\begin{array}{lllll}
0 & 0 & 0 & 0 & 1 \\
0 & 1 & 0 & 0 & 0 \\
0 & 0 & 1 & 0 & 0 \\
0 & 0 & 0 & 1 & 0 \\
1 & 0 & 0 & 0 & 0
\end{array}\right)
$$

and since $\operatorname{det}(V)=-1$, we conclude that $\mathbb{X}$ is a strict Gorenstein scheme.

The following example of a strict Gorenstein scheme is the well-known case of eight points on a twisted cubic curve. Notice that in [15, Example 4.6, we check that it is not a strict complete intersection.

Example 7.7. Let $K=\mathbb{Q}$, let $P=K[x, y, z]$, and let $\mathbb{X}$ be the reduced subscheme of $\mathbb{A}_{K}^{3}$ consisting of the eight points $p_{1}=(0,0,0), p_{2}=(1,1,1), p_{3}=(-1,1,-1)$, $p_{4}=(2,4,8), p_{5}=(-2,4,-8), p_{6}=(3,9,27), p_{7}=(-3,9,-27)$, and $p_{8}=$ $(4,16,64)$ on the twisted cubic curve $T=\left\{\left(t, t^{2}, t^{3}\right) \in \mathbb{A}_{K}^{3} \mid t \in K\right\}$.

The reduced Gröbner basis with respect to the DegRevLex term ordering such 
that $z>y>x$ of the vanishing ideal $I_{\mathbb{X}}$ is

$$
\begin{aligned}
& \left\{x^{2}-y, x y-z, y^{2}-x z, y z^{2}-4 x z^{2}-14 z^{2}+56 y z+49 x z-196 z-36 y+144 x,\right. \\
& \left.z^{3}-30 x z^{2}+273 y z-820 z+576 x\right\}
\end{aligned}
$$

and hence we have $\operatorname{DF}\left(I_{\mathbb{X}}\right)=\left\langle x^{2}, x y, y^{2}-x z, y z^{2}-4 x z^{2}, z^{3}-30 x z^{2}\right\rangle$.

Now we apply Algorithm 7.4 To get a degree filtered $K$-basis of $R_{\mathbb{X}}$, it suffices to take the terms $\mathcal{O}=\left\{1, x, y, z, x z, y z, z^{2}, x z^{2}\right\}$ in the complement of the leading term ideal of $I_{\mathbb{X}}$. Then the Hilbert function of $\mathcal{O}$ is $(1,3,3,1)$, and we observe that it is symmetric.

Next we determine the multiplication matrices corresponding to the multiplication by the elements of $\mathcal{O}$ on $P / D F\left(I_{\mathbb{X}}\right)$ and combine their last columns to get

$$
V=\left(\begin{array}{cccccccc}
0 & 0 & 0 & 0 & 0 & 0 & 0 & 1 \\
0 & 0 & 0 & 0 & 0 & 0 & 1 & 0 \\
0 & 0 & 0 & 0 & 0 & 1 & 4 & 0 \\
0 & 0 & 0 & 0 & 1 & 4 & 30 & 0 \\
0 & 0 & 0 & 1 & 0 & 0 & 0 & 0 \\
0 & 0 & 1 & 4 & 0 & 0 & 0 & 0 \\
0 & 1 & 4 & 30 & 0 & 0 & 0 & 0 \\
1 & 0 & 0 & 0 & 0 & 0 & 0 & 0
\end{array}\right)
$$

Since $\operatorname{det}(V)=1 \neq 0$, it follows that $\mathbb{X}$ is a strict Gorenstein scheme.

\subsection{Computing the Strict Cayley-Bacharach Locus}

In the following we present algorithms to calculate the loci of all strict CayleyBacharach schemes and all strict Gorenstein schemes in $\mathbb{B}_{\mathcal{O}}^{\text {df }}$. We use the following terminology.

Definition 7.8. Let $\mathcal{O}=\left\{t_{1}, \ldots, t_{\mu}\right\}$ be an order ideal in $\mathbb{T}^{n}$.

(a) The set of all $K$-rational points $\Gamma=\left(\gamma_{i j}\right) \in K^{\mu \nu}$ of the border basis scheme $\mathbb{B}_{\mathcal{O}}$ which represent a 0-dimensional strict Cayley-Bacharach scheme $\mathbb{X}_{\Gamma}$ is called the set of strict Cayley-Bacharach points of $\mathbb{B}_{\mathcal{O}}$.

(b) The set of all $K$-rational points $\Gamma=\left(\gamma_{i j}\right) \in K^{\mu \nu}$ of the border basis scheme $\mathbb{B}_{\mathcal{O}}$ which represent a 0 -dimensional strict Gorenstein scheme $\mathbb{X}_{\Gamma}$ is called the set of strict Gorenstein points of $\mathbb{B}_{\mathcal{O}}$.

Using the next algorithm, we see that there exists an open subscheme $\mathrm{SCB}_{\mathcal{O}}^{\mathrm{df}}$ of $\mathbb{B}_{\mathcal{O}}^{\text {df }}$ whose $K$-rational points are precisely the strict Cayley-Bacharach points of $\mathbb{B}_{\mathcal{O}}$ which are contained in $\mathbb{B}_{\mathcal{O}}^{\text {df }}$. This scheme is called the strict CayleyBacharach locus in $\mathbb{B}_{\mathcal{O}}^{\mathrm{df}}$. We need the following method for finding the generic multiplication matrices on the associated graded rings.

Remark 7.9. Let $\mathcal{O}=\left\{t_{1}, \ldots, t_{\mu}\right\}$ be an order ideal in $\mathbb{T}^{n}$, let $\partial \mathcal{O}=\left\{b_{1}, \ldots, b_{\nu}\right\}$ be its border, and let $G^{\mathrm{df}}=\left\{g_{1}^{\mathrm{df}}, \ldots, g_{\nu}^{\mathrm{df}}\right\}$ be the generic degree filtered $\mathcal{O}$-border 
prebasis. For every $K$-rational point $\Gamma=\left(\gamma_{i j}\right)$ of $\mathbb{B}_{\mathcal{O}}^{\text {df }}$, the 0 -dimensional scheme $\mathbb{X}_{\Gamma}$ has the associated graded ring $\operatorname{gr}_{\mathcal{F}}\left(R_{\mathbb{X}_{\Gamma}}\right) \cong P / \mathrm{DF}\left(I_{\mathbb{X}_{\Gamma}}\right)$. Since $\mathbb{X}_{\Gamma}$ has a degree filtered $\mathcal{O}$-border basis, we have $\operatorname{DF}\left(I_{\mathbb{X}_{\Gamma}}\right)=\left\langle\mathrm{DF}\left(g_{1}\right), \ldots, \mathrm{DF}\left(g_{\nu}\right)\right\rangle$, where $\operatorname{DF}\left(g_{j}\right)=$ $b_{j}-\sum_{\left\{i \mid \operatorname{deg}\left(t_{i}\right)=\operatorname{deg}\left(b_{j}\right)\right\}} \gamma_{i j} t_{i}$ (see [20, Theorem 2.4).

Therefore all these associated graded rings are parametrized by the $K$-algebra $U_{\mathcal{O}}^{\text {hom }}=B_{\mathcal{O}}^{\text {hom }}\left[x_{1}, \ldots, x_{n}\right] /\left\langle g_{1}^{\text {hom }}, \ldots, g_{\nu}^{\text {hom }}\right\rangle$, where $B_{\mathcal{O}}^{\text {hom }}$ is the affine coordinate ring of the homogeneous border basis scheme, and where we have the equality $g_{j}^{\text {hom }}=b_{j}-\sum_{\left\{i \mid \operatorname{deg}\left(t_{i}\right)=\operatorname{deg}\left(b_{j}\right)\right\}} c_{i j} t_{i}$ for $j=1, \ldots, \nu$. Thus the multiplication matrices of the associated graded rings are parametrized by the generic homogeneous multiplication matrices $\mathcal{A}_{1}^{\text {hom }}, \ldots, \mathcal{A}_{n}^{\text {hom }}$ given in Remark 5.11] For an arbitrary element $f \in P$, the multiplication by $f$ of the associated graded rings is therefore given by $M_{f}^{\text {hom }}=f\left(\mathcal{A}_{1}^{\text {hom }}, \ldots, \mathcal{A}_{n}^{\text {hom }}\right)$ with respect to the basis $\mathcal{O}$.

Thus we are ready to compute the following subscheme of $\mathbb{B}_{\mathcal{O}}^{\mathrm{df}}$.

Algorithm 7.10. (Computing the Strict Cayley-Bacharach Locus in $\mathbb{B}_{\mathcal{O}}^{\mathrm{df}}$ ) Let $\mathcal{O}=\left\{t_{1}, \ldots, t_{\mu}\right\}$ be an order ideal in $\mathbb{T}^{n}$, and let $\Delta=\#\{i \in\{1, \ldots, \mu\} \mid$ $\left.\operatorname{deg}\left(t_{i}\right)=\operatorname{deg}\left(t_{\mu}\right)\right\}$. Consider the following sequence of instructions.

(1) Using Proposition 5.3. b, calculate $I\left(\mathbb{B}_{\mathcal{O}}^{\mathrm{df}}\right)=I\left(\mathbb{B}_{\mathcal{O}}\right)+I_{\mathcal{O}}^{\mathrm{df}}$.

(2) For $i=1, \ldots, \mu$ use Remark 7.9 to compute the multiplication matrix $M_{t_{i}}^{\text {hom }}$ for the multiplication by $t_{i}$ on $U_{\mathcal{O}}^{\text {hom }}$.

(3) For $j=1, \ldots, \Delta$, form the matrix $V_{j} \in \operatorname{Mat}_{\mu}(K[C])$ whose $i$-th column is the $(\mu-\Delta+j)$-th column of $\left(M_{t_{i}}^{\text {hom }}\right)^{\text {tr }}$ for $i=1, \ldots, \mu$.

(4) Form the block column matrix $W=\operatorname{Col}\left(V_{1}, \ldots, V_{\Delta}\right)$ and compute the ideal $J$ in $K[C]$ generated by the maximal minors of $W$.

(5) Return the ideal $I\left(\mathbb{B}_{\mathcal{O}}^{\mathrm{df}}\right)+J$.

This is an algorithm which computes an ideal in the ring $K[C]$. This ideal defines a closed subscheme NonSCB $\mathrm{df}_{\mathcal{O}}^{\mathrm{df}}$ whose $K$-rational points represent the 0-dimensional subschemes of $\mathbb{A}_{K}^{n}$ which have a degree filtered $\mathcal{O}$-border basis, but are not strict Cayley-Bacharach schemes.

Proof. A $K$-rational rational point $\Gamma=\left(\gamma_{i j}\right)$ of $\mathbb{B}_{\mathcal{O}}^{\text {df }}$ corresponds to a zero of the ideal $I\left(\mathbb{B}_{\mathcal{O}}^{\text {df }}\right)$ which is computed in Step (1). By Algorithm 7.2, the 0-dimensional scheme $\mathbb{X}_{\Gamma}$ represented by $\Gamma$ is a strict Cayley-Bacharach scheme if and only if $\Gamma$ is a zero of the ideal generated by the maximal minors of $W$, and this observation finishes the proof.

In view of this algorithm, we see that $\mathrm{SCB}_{\mathcal{O}}^{\mathrm{df}}=\mathbb{B}_{\mathcal{O}}^{\mathrm{df}} \backslash \operatorname{NonSCB}_{\mathcal{O}}^{\mathrm{df}}$ is an open subscheme of $\mathbb{B}_{\mathcal{O}}^{\mathrm{df}}$. Let us calculate the scheme $\operatorname{NonSCB}_{\mathcal{O}}^{\mathrm{df}}$ in the setting of Example 6.4.

Example 7.11. Let $\mathcal{O}$ be the order ideal $\mathcal{O}=\left\{1, x, y, z, x^{2}\right\}$ in $P=K[x, y, z]$. We apply Algorithm 7.10 to compute the ideal defining the subscheme NonSCB ${ }_{\mathcal{O}}^{\text {df }}$ of $\mathbb{B}_{\mathcal{O}}^{\text {df }}$ and get $I\left(\mathbb{B}_{\mathcal{O}}^{\mathrm{df}}\right)+\left\langle c_{52} c_{53}^{2}-2 c_{51} c_{53} c_{54}+c_{51}^{2} c_{55}+c_{54}^{2}-c_{52} c_{55}\right\rangle$. Hence the 
strict Cayley-Bacharach locus and the Cayley-Bacharach locus in $\mathbb{B}_{\mathcal{O}}^{\text {df }}$ are identical in this case. This is in agreement with [14, Theorems 6.8 and 6.12, since the Hilbert function of $\mathcal{O}$ is symmetric and satisfies $\Delta=1$.

In the following example, the Cayley-Bacharach and the strict Cayley-Bacharach locus differ.

Example 7.12. Consider the order ideal $\mathcal{O}=\left\{1, x, y, x^{2}, x y, y^{2}, x^{3}\right\}$ in $P=$ $K[x, y]$. Its border is $\partial \mathcal{O}=\left\{x^{2} y, x y^{2}, y^{3}, x^{4}, x^{3} y\right\}$. Thus we have $\mu=7, \nu=5$, and the ideal $I\left(\mathbb{B}_{\mathcal{O}}\right)$ has 28 generators. Since the order ideal $\mathcal{O}$ has the generic Hilbert function, we get $I\left(\mathbb{B}_{\mathcal{O}}^{\text {df }}\right)=I\left(\mathbb{B}_{\mathcal{O}}\right)$ by Proposition 5.8 .

Now we use Algorithm 6.3 to compute an ideal which defines $\mathrm{NonCB}_{\mathcal{O}}^{\mathrm{df}}$. We obtain the ideal $I\left(\mathbb{B}_{\mathcal{O}}\right)+\langle f\rangle$ where $f \in K[C]$ is a polynomial of the shape

$$
f=c_{43} c_{71}^{5}-c_{53} c_{71}^{4} c_{72}+\cdots+c_{42} c_{73}^{2}
$$

Thus the Cayley-Bacharach locus in $\mathbb{B}_{\mathcal{O}}^{\mathrm{df}}$ is the complement of a hypersurface section of $\mathbb{B}_{\mathcal{O}}^{\mathrm{df}}$.

Next we use Algorithm 7.10 to compute an ideal which defines $\operatorname{NonSCB}_{\mathcal{O}}^{\mathrm{df}}$. The result is the ideal $I\left(\mathbb{B}_{\mathcal{O}}\right)$, in agreement with 14 , Thm. 6.12, which says that every strict Cayley-Bacharach scheme with $\Delta_{\mathbb{X}}=1$ is a strict Gorenstein scheme. Since the Hilbert function $(1,2,3,1)$ of $\mathcal{O}$ is not symmetric, this is impossible here. In other words, the strict Cayley-Bacharach locus in $\mathbb{B}_{\mathcal{O}}^{\mathrm{df}}$ is empty.

Finally, we combine Algorithm 7.10 with a check for the symmetry of the Hilbert function of $\mathcal{O}$ and get the following corollary. It shows that there exists an open subscheme SGor $_{\mathcal{O}}^{\mathrm{df}}$ of $\mathbb{B}_{\mathcal{O}}^{\mathrm{df}}$ whose $K$-rational points are exactly those points in the set of strict Gorenstein points of $\mathbb{B}_{\mathcal{O}}$ which are contained in $\mathbb{B}_{\mathcal{O}}^{\mathrm{df}}$. This subscheme is called the strict Gorenstein locus in $\mathbb{B}_{\mathcal{O}}^{\mathrm{df}}$.

Corollary 7.13. (Computing the Strict Gorenstein Locus in $\mathbb{B}_{\mathcal{O}}^{\mathrm{df}}$ )

Let $\mathcal{O}=\left\{t_{1}, \ldots, t_{\mu}\right\}$ be an order ideal in $\mathbb{T}^{n}$, and let $\varrho=\operatorname{deg}\left(t_{\mu}\right)$. Then the following instructions define an algorithm which computes an ideal in $K[C]$. This ideal defines a closed subscheme NonSGor ${ }_{\mathcal{O}}^{\mathrm{df}}$ whose $K$-rational points represent the 0 -dimensional subschemes of $\mathbb{A}_{K}^{n}$ which have a degree filtered $\mathcal{O}$-border basis, but are not strict Gorenstein schemes.

(1) Using Proposition 5.3. b, calculate $I\left(\mathbb{B}_{\mathcal{O}}^{\mathrm{df}}\right)=I\left(\mathbb{B}_{\mathcal{O}}\right)+I_{\mathcal{O}}^{\mathrm{df}}$.

(2) If the Hilbert function $\mathrm{HF}_{\mathcal{O}}$ is not symmetric, then return the ideal $I\left(\mathbb{B}_{\mathcal{O}}^{\mathrm{df}}\right)$ and stop.

(3) Otherwise, apply Algorithm 7.10 and return the ideal $I\left(\mathbb{B}_{\mathcal{O}}^{\mathrm{df}}\right)+J$ it computes.

Proof. A $K$-rational rational point $\Gamma=\left(\gamma_{i j}\right)$ of $\mathbb{B}_{\mathcal{O}}^{\text {df }}$ corresponds to a zero of the ideal $I\left(\mathbb{B}_{\mathcal{O}}^{\mathrm{df}}\right)$ which is computed in Step (1). If the corresponding associated graded ring $\operatorname{gr}_{\mathcal{F}}\left(R_{\mathbb{X}_{\Gamma}}\right) \cong P / \operatorname{DF}\left(I_{\mathbb{X}_{\Gamma}}\right)$ is to be a Gorenstein ring, its Hilbert function $\left(h_{0}, \ldots, h_{\mu}\right)$ has to be symmetric. Therefore Step (2) correctly returns the ideal of $\mathbb{B}_{\mathcal{O}}^{\mathrm{df}}$ if that Hilbert function is not symmetric. 
Now assume that the Hilbert function is symmetric. By Algorithm 7.4 we know that the 0 -dimensional scheme $\mathbb{X}_{\Gamma}$ represented by $\Gamma$ is a strict Gorenstein scheme if and only if $\Gamma$ is a zero of the ideal $I\left(\mathbb{B}_{\mathcal{O}}^{\mathrm{df}}\right)+J$, and this observation finishes the proof.

Let us complete the discussion of Corollary 7.13 by the following observation.

Remark 7.14. Suppose that $\mathcal{O}=\left\{t_{1}, \ldots, t_{\mu}\right\}$ is an order ideal such that only one term has the maximal degree $\max \left\{\operatorname{deg}\left(t_{1}\right), \ldots, \operatorname{deg}\left(t_{\mu}\right)\right\}$. Then [14, Thm. 6.12 implies NonSGor Nf $_{\mathcal{O}}^{\mathrm{df}}=$ NonSCB $_{\mathcal{O}}^{\mathrm{df}}$. Hence we may use either Algorithm[7.10 or Corollary 7.13 to compute this subscheme.

In the setting of Example 6.4, the Hilbert function of $\mathcal{O}=\left\{1, x, y, z, x^{2}\right\}$ is symmetric, and therefore the ideals defining the closed subschemes $\operatorname{NonSCB}_{\mathcal{O}}^{\mathrm{df}}$ and NonSGor ${ }_{\mathcal{O}}^{\mathrm{df}}$ of $\mathbb{B}_{\mathcal{O}}^{\mathrm{df}}$ are identical. On the other hand, in the setting of Example 7.12, the Hilbert function of $\mathcal{O}=\left\{1, x, y, x^{2}, x y, y^{2}, x^{3}\right\}$ is not symmetric and Corollary 7.13 returns an ideal which defines $\mathbb{B}_{\mathcal{O}}^{\text {df }}$.

\section{The Strict Complete Intersection Locus in $\mathbb{B}_{\mathcal{O}}^{\mathrm{df}}$}

Recall that a 0 -dimensional ring of the form $K\left[x_{1}, \ldots, x_{n}\right] / I$ with a field $K$ and a homogeneous 0 -dimensional ideal $I$ is called a graded complete intersection if $I$ can be generated by a homogeneous regular sequence of length $n$. In our setting, the following version of this notion will be considered.

Definition 8.1. Let $\mathbb{X}$ be a 0 -dimensional subscheme of $\mathbb{A}_{K}^{n}$, let $I_{\mathbb{X}}$ be the vanishing ideal of $\mathbb{X}$ in $P$, let $R_{\mathbb{X}}$ be the affine coordinate ring of $\mathbb{X}$, and let $\mathcal{F}$ be the degree filtration of $R_{\mathbb{X}}$.

Then the scheme $\mathbb{X}$ is called a strict complete intersection scheme if the associated graded ring $\operatorname{gr}_{\mathcal{F}}\left(R_{\mathbb{X}}\right) \cong P / \mathrm{DF}\left(I_{\mathbb{X}}\right)$ is a graded complete intersection.

Various equivalent conditions and properties of this notion are discussed in [15], Sections 4 and 5. In particular, Algorithm 5.4 of [15] provides a way to check whether $\mathbb{X}$ is a strict complete intersection scheme which uses the knowledge of a degree filtered border basis.

This brings us to the topic of this section: to compute the locus of strict complete intersection schemes inside the degree filtered border basis scheme. We begin again by fixing the terminology.

Definition 8.2. Let $\mathcal{O}=\left\{t_{1}, \ldots, t_{\mu}\right\}$ be an order ideal in $\mathbb{T}^{n}$. Then the set of all $K$-rational points $\Gamma=\left(\gamma_{i j}\right) \in K^{\mu \nu}$ of the border basis scheme $\mathbb{B}_{\mathcal{O}}$ which represent a 0 -dimensional strict complete intersection scheme $\mathbb{X}_{\Gamma}$ is called the set of strict complete intersection points of $\mathbb{B}_{\mathcal{O}}$.

The following algorithm computes a closed subscheme $\operatorname{NonSCI}_{\mathcal{O}}^{\mathrm{df}}$ of $\mathbb{B}_{\mathcal{O}}^{\mathrm{df}}$ such that the $K$-rational points of the open subscheme $\operatorname{SCI}_{\mathcal{O}}^{\mathrm{df}}=\mathbb{B}_{\mathcal{O}}^{\mathrm{df}} \backslash$ NonSCI $\mathrm{O}_{\mathcal{O}}^{\mathrm{df}}$ are 
precisely the strict complete intersection points in $\mathbb{B}_{\mathcal{O}}^{\mathrm{df}}$. This open subscheme is called the strict complete intersection locus in $\mathbb{B}_{\mathcal{O}}^{\mathrm{df}}$.

\section{Algorithm 8.3. (Computing the Strict CI Locus in $\mathbb{B}_{\mathcal{O}}^{\mathrm{df}}$ )}

Let $\mathcal{O}=\left\{t_{1}, \ldots, t_{\mu}\right\}$ be an order ideal in $\mathbb{T}^{n}$, and let $\varrho=\operatorname{deg}\left(t_{\mu}\right)$. Consider the following sequence of instructions.

(1) Using Proposition 5.3, b, calculate $I\left(\mathbb{B}_{\mathcal{O}}^{\mathrm{df}}\right)=I\left(\mathbb{B}_{\mathcal{O}}\right)+I_{\mathcal{O}}^{\mathrm{df}}$.

(2) If the Hilbert function $\mathrm{HF}_{\mathcal{O}}$ of $\mathcal{O}$ is not symmetric, then return the ideal $I\left(\mathbb{B}_{\mathcal{O}}^{\mathrm{df}}\right)$ and stop.

(3) Form the generic homogeneous $\mathcal{O}$-border prebasis $G^{\text {hom }}=\left\{g_{1}^{\text {hom }}, \ldots, g_{j}^{\text {hom }}\right\}$ and write $g_{j}^{\text {hom }}=\sum_{i=1}^{n} h_{i j} x_{i}$ with $h_{i j} \in K[C]\left[x_{1}, \ldots, x_{n}\right]$ for $j=1, \ldots, \nu$.

(4) Form the matrix $W$ of size $n \times \nu$ whose columns are given by $\left(h_{1 j}, \ldots, h_{n j}\right)^{\operatorname{tr}}$ for $j=1, \ldots, \nu$.

(5) Let $k=\left(\begin{array}{l}\nu \\ n\end{array}\right)$. Calculate the minors $f_{1}, \ldots, f_{k}$ of order $n$ of $W$.

(6) Using border division by $G^{\text {hom }}$, write the residue classes $\bar{f}_{1}, \ldots, \bar{f}_{k} \in U_{\mathcal{O}}^{\text {hom }}$ as $B_{\mathcal{O}}^{\text {hom }}$-linear combinations $\bar{f}_{j}=\sum_{i=1}^{\mu} \bar{a}_{i j} t_{i}$ with $\bar{a}_{1 j}, \ldots, \bar{a}_{\mu j} \in B_{\mathcal{O}}^{\text {hom }}$ for $j=1, \ldots, k$.

(7) Let $C^{\text {hom }}=\left\{c_{i j} \mid \operatorname{deg}\left(t_{i}\right)=\operatorname{deg}\left(b_{j}\right)\right\}$. For $i=1, \ldots, \mu$ and $j=1, \ldots, k$, choose $a_{i j} \in K\left[C^{\text {hom }}\right]$ which represents $\bar{a}_{i j}$ with respect to $B_{\mathcal{O}}^{\text {hom }} \cong K\left[C^{\text {hom }}\right] / \bar{I}\left(\mathbb{B}_{\mathcal{O}}^{\text {hom }}\right)$. Return the ideal $J=I\left(\mathbb{B}_{\mathcal{O}}^{\mathrm{df}}\right)+\left\langle a_{i j} \mid i \in\{1, \ldots, \mu\}, j \in\{1, \ldots, k\}\right\rangle$ and stop.

This is an algorithm which computes an ideal $J$ in the ring $K[C]$ which defines a closed subscheme $\mathrm{NonSCI}_{\mathcal{O}}^{\mathrm{df}}$ of $\mathbb{B}_{\mathcal{O}}^{\mathrm{df}}$. The $K$-rational points of this subscheme represent the 0-dimensional subschemes of $\mathbb{A}_{K}^{n}$ which have a degree filtered $\mathcal{O}$ border basis, but are not strict complete intersection schemes.

Proof. In Step (1) we calculate the ideal defining $\mathbb{B}_{\mathcal{O}}^{\mathrm{df}}$. In Step (2) we check whether the Castelnuovo function of a 0 -dimensional scheme $\mathbb{X}_{\Gamma}$ represented by a $K$-rational point $\Gamma$ of $\mathbb{B}_{\mathcal{O}}^{\text {df }}$ is symmetric. This is certainly a necessary condition, because strict complete intersections are strict Gorenstein schemes. If it is not satisfied, we return the ideal of $\mathbb{B}_{\mathcal{O}}^{\text {df }}$.

Since the ideal $J$ returned by the algorithm contains the ideal of $\mathbb{B}_{\mathcal{O}}^{\text {df }}$, we clearly compute a closed subscheme of $\mathbb{B}_{\mathcal{O}}^{\text {df }}$. Notice that the ideal $J$ does not depend on the choice of the representatives $a_{i j}$ in Step (7), since the elements of $\bar{I}\left(\mathbb{B}_{\mathcal{O}}^{\text {hom }}\right)$ are contained in $I\left(\mathbb{B}_{\mathcal{O}}^{\mathrm{df}}\right)$ by Proposition 5.13 .

A $K$-rational point $\Gamma$ of $\mathbb{B}_{\mathcal{O}}^{\text {df }}$ is a zero of the ideal $J$ returned by the algorithm if and only if the vanishing ideal $I_{\mathbb{X}_{\Gamma}}$ satisfies the conditions required in Algorithm 5.4 of [15. Hence the scheme $\mathbb{X}_{\Gamma}$ is not a strict complete intersection if and only if the point $\Gamma$ is a zero of the ideal $J$, as was to be shown.

In the following we illustrate this algorithm with a couple of examples.

Example 8.4. As in Example 3.6, let $\mathcal{O}$ be the order ideal $\mathcal{O}=\{1, x, y, x y\}$ in $P=K[x, y]$. Then we have $\partial \mathcal{O}=\left\{x^{2}, y^{2}, x^{2} y, x y^{2}\right\}$, and thus $\mu=\nu=4$. The ideal 
$I\left(\mathbb{B}_{\mathcal{O}}\right)=I\left(\mathbb{B}_{\mathcal{O}}^{\text {df }}\right)$ was determined in Examples 3.6 and 5.14.a. It is generated by 12 quadratic equations in 16 indeterminates.

Since the Hilbert function $(1,2,1,0, \ldots)$ of $\mathcal{O}$ is symmetric, we can use Corollary 7.13 to compute the ideal defining the closed subscheme NonSGor ${ }_{\mathcal{O}}^{\mathrm{df}}$ of $\mathbb{B}_{\mathcal{O}}^{\mathrm{df}}$ and get $I\left(\mathbb{B}_{\mathcal{O}}^{\text {df }}\right)+\left\langle c_{41} c_{42}-1\right\rangle$.

Next we apply Algorithm 8.3 to compute the ideal defining $\operatorname{NonSCI}_{\mathcal{O}}^{\mathrm{df}}$. The matrix $W$ in Step (4) is

$$
W=\left(\begin{array}{cccc}
-x_{2} c_{41}+x_{1} & -x_{2} c_{42} & x_{1} x_{2} & x_{2}^{2} \\
0 & x_{2} & 0 & 0
\end{array}\right)
$$

This matrix has three non-zero maximal minors, namely $f_{1}=-x_{2}^{2} c_{41}+x_{1} x_{2}$, $f_{2}=-x_{1} x_{2}^{2}$, and $f_{3}=-x_{2}^{3}$. In Step (6) we apply border division by

$$
G^{\text {hom }}=\left\{x_{1}^{2}-x_{1} x_{2} c_{41}, x_{2}^{2}-x_{1} x_{2} c_{42}, x_{1}^{2} x_{2}, x_{1} x_{2}^{2}\right\}
$$

to these polynomials. Only the division of $f_{1}$ by $G^{\text {hom }}$ yields a non-zero result, namely $x_{1} x_{2}\left(-c_{41} c_{42}+1\right)$. Consequently, the algorithm returns the ideal $I\left(\mathbb{B}_{\mathcal{O}}\right)+$ $\left\langle c_{41} c_{42}-1\right\rangle$ in Step (7).

Thus the ideals which define NonSGor ${ }_{\mathcal{O}}^{\mathrm{df}}$ and $\operatorname{NonSCI}_{\mathcal{O}}^{\mathrm{df}}$ are identical. This is in agreement with the fact that in codimension 2 the notions of a Gorenstein ring and a complete intersection are equivalent.

In order to find a case where the loci of strict Gorenstein and strict complete intersection scheme differ, we have to consider 0 -dimensional schemes in $\mathbb{A}_{K}^{n}$ with $n \geq 3$. The following case is one of the easiest ones.

Example 8.5. Consider the order ideal $\mathcal{O}=\left\{1, x, y, z, x^{2}, x y, x z, x^{3}\right\}$ in $P=$ $K[x, y, z]$. Its Hilbert function $\mathrm{HF}_{\mathcal{O}}=(1,3,3,1,0, \ldots)$ is symmetric. The border of $\mathcal{O}$ is $\partial \mathcal{O}=\left\{y^{2}, y z, z^{2}, x^{2} y, x^{2} z, x y^{2}, x y z, x z^{2}, x^{4}, x^{3} y, x^{3} z\right\}$. Consequently, we have $\mu=8$ and $\nu=11$, and the ring $K[C]$ has 88 indeterminates. Using Proposition $5.3 \mathrm{~b}$, we calculate $I\left(\mathbb{B}_{\mathcal{O}}^{\mathrm{df}}\right)=I\left(\mathbb{B}_{\mathcal{O}}\right)+I_{\mathcal{O}}^{\mathrm{df}}$. The resulting ideal has 147 generators, 3 of which are indeterminates, and the remaining 144 are quadratic polynomials.

Using Corollary 7.13, we compute an ideal which defines NonSGor df $_{\mathcal{O}}$ and get $I\left(\mathbb{B}_{\mathcal{O}}^{\text {df }}\right)+\langle f\rangle$, where $f$ is a polynomial of the form $f=g^{2}$ with

$$
g=c_{85}^{2} c_{86}-2 c_{84} c_{85} c_{87}+c_{48}^{2} c_{88}+c_{87}^{2}-c_{86} c_{88}
$$

Thus the strict Gorenstein locus in $\mathbb{B}_{\mathcal{O}}^{\mathrm{df}}$ is the complement of the hypersurface section defined by $f$. Notice that the polynomials $f$ and $g$ are homogeneous of arrow degree zero.

Next we apply Algorithm 8.3 to calculate an ideal which defines NonSCI $_{\mathcal{O}}^{\text {df }}$. The matrix $W$ in Step (4) is

$$
W=\left(\begin{array}{ccccccccccc}
p_{1} & p_{2} & p_{3} & p_{4} & p_{5} & p_{6} & p_{7} & p_{8} & x_{1}^{3} & x_{1}^{2} x_{2} & x_{1}^{2} x_{3} \\
x_{2} & x_{3} & 0 & 0 & 0 & 0 & 0 & 0 & 0 & 0 & 0 \\
0 & 0 & x_{3} & 0 & 0 & 0 & 0 & 0 & 0 & 0 & 0
\end{array}\right)
$$


where $p_{1}=-x_{1} c_{51}-x_{2} c_{61}-x_{3} c_{71}, p_{2}=-x_{1} c_{52}-x_{2} c_{62}-x_{3} c_{72}, p_{3}=-x_{1} c_{53}-$ $x_{2} c_{63}-x_{3} c_{73}, p_{4}=-x_{1}^{2} c_{84}+x_{1} x_{2}, p_{5}=-x_{1}^{2} c_{85}+x_{1} x_{3}, p_{6}=-x_{1}^{2} c_{86}+x_{2}^{2}$, $p_{7}=-x_{1}^{2} c_{87}+x_{2} x_{3}$, and $p_{8}=-x_{1}^{2} c_{88}+x_{3}^{2}$. This matrix has 17 non-zero maximal minors, and only one of them yields a non-zero remainder after division by $G^{\text {hom }}$, namely $h \cdot x^{3}$, where $h$ is the polynomial

$$
\begin{aligned}
h= & -c_{52} c_{61} c_{85}+c_{51} c_{62} c_{85}-c_{53} c_{71} c_{85}+c_{52} c_{72} c_{85}-c_{63} c_{71} c_{87}+c_{62} c_{72} c_{87} \\
& +c_{62} c_{71} c_{88}-c_{61} c_{72} c_{88}+c_{72}^{2} c_{88}-c_{71} c_{73} c_{88}+c_{52} c_{87}-c_{51} c_{88}
\end{aligned}
$$

Altogether, the result is the ideal $I\left(\mathbb{B}_{\mathcal{O}}^{\mathrm{df}}\right)+\langle h\rangle$ which defines NonSCI $\mathrm{N}_{\mathcal{O}}^{\mathrm{df}}$. At this point we may check that $I\left(\mathbb{B}_{\mathcal{O}}^{\text {df }}\right)+\langle f\rangle \subsetneq I\left(\mathbb{B}_{\mathcal{O}}^{\text {df }}\right)+\langle g\rangle=I\left(\mathbb{B}_{\mathcal{O}}^{\text {df }}\right)+\langle h\rangle$. This shows that the locus of strict complete intersections in $\mathbb{B}_{\mathcal{O}}^{\mathrm{df}}$ is properly contained in the locus of strict Gorenstein schemes in $\mathbb{B}_{\mathcal{O}}^{\mathrm{df}}$. As Example 7.7 and [15], Example 4.6 indicate, eight points on a suitably chosen twisted cubic curve yield a point in the strict Gorenstein locus of $\mathbb{B}_{\mathcal{O}}^{\mathrm{df}}$ which is not contained in the strict complete intersection locus.

\section{The Hilbert Stratification of $\mathbb{B}_{\mathcal{O}}$}

In general, the border basis scheme $\mathbb{B}_{\mathcal{O}}$ contains $K$-rational points which represent 0-dimensional schemes having different affine Hilbert functions. In this section we describe and calculate the stratification of $\mathbb{B}_{\mathcal{O}}$ determined by these affine Hilbert functions.

As in the preceding sections, let $K$ be a field, let $P=K\left[x_{1}, \ldots, x_{n}\right]$, and let $\mathcal{O}=\left\{t_{1}, \ldots, t_{\mu}\right\}$ be an order ideal in $\mathbb{T}^{n}$ with border $\partial \mathcal{O}=\left\{b_{1}, \ldots, b_{\nu}\right\}$. Recall that we always order $\mathcal{O}$ such that $\operatorname{deg}\left(t_{1}\right) \leq \cdots \leq \operatorname{deg}\left(t_{\mu}\right)$. In particular, we have $t_{1}=1$ (see Assumption 2.2). In this setting, we introduce the following terminology (see also Definition 2.3).

Definition 9.1. Given a $K$-rational point $\Gamma=\left(\gamma_{i j}\right)$ of $\mathbb{B}_{\mathcal{O}}$, let $G_{\Gamma}$ be the corresponding border basis, and let $R_{\Gamma}=P /\left\langle G_{\Gamma}\right\rangle$ be the affine coordinate ring of the 0 -dimensional affine scheme $\mathbb{X}_{\Gamma}$ represented by $\Gamma$.

(a) The affine Hilbert function of $R_{\Gamma}$ is called the affine Hilbert function associated to $\Gamma$. The values of the affine Hilbert function associated to $\Gamma$ will be denoted by

$$
\mathrm{HF}_{R_{\Gamma}}^{a}=\left(H_{0}, H_{1}, H_{2}, \ldots\right)
$$

where $H_{i}=\operatorname{HF}_{R_{\Gamma}}^{a}(i)$ for $i \geq 0$.

(b) The values of the Castelnuovo function of $R_{\Gamma}$ will be denoted by

$$
\Delta \mathrm{HF}_{R_{\Gamma}}^{a}=\left(h_{0}, h_{1}, h_{2}, \ldots\right)
$$

where $h_{0}=1$ and $h_{i}=H_{i}-H_{i-1}$ for $i \geq 1$. 
(c) We say that a sequence of non-negative integers $\left(H_{0}, H_{1}, \ldots\right)$ dominates a sequence of non-negative integers $\left(H_{0}^{\prime}, H_{1}^{\prime}, \ldots\right)$ if we have $H_{i} \geq H_{i}^{\prime}$ for all $i \geq 0$. In this case we also write $\left(H_{0}, H_{1}, \ldots\right) \succeq\left(H_{0}^{\prime}, H_{1}^{\prime}, \ldots\right)$.

(d) Given $n, \mu \in \mathbb{N}_{+}$, a sequence $\mathcal{H}=\left(H_{0}, H_{1}, \ldots\right)$ of non-negative integers is called $(n, \mu)$-admissible if there exists a 0 -dimensional affine subscheme $\mathbb{X}$ in $\mathbb{A}_{K}^{n}$ of length $\mu$ such that $\mathcal{H}=\mathrm{HF}_{\mathbb{X}}^{a}$.

(e) Given $n, \mu \in \mathbb{N}_{+}$, the sequence $\mathrm{HF}^{(n, \mu)}=\left(H_{0}, H_{1}, \ldots\right)$ such that we have $H_{i}=\min \left\{\mu, \operatorname{HF}_{P}^{a}(i)\right\}$ for all $i \geq 0$ is called the generic affine Hilbert function for a scheme of length $\mu$ in $\mathbb{A}_{K}^{n}$.

In 18, Section 5.5, precise formulas are given which characterize admissible Hilbert functions. Also, note that from [18, Theorem 5.5.32, it follows that $\mathrm{HF}^{(n, \mu)}$ is admissible.

Proposition 9.2. Let $\Gamma$ be a $K$-rational point of $\mathbb{B}_{\mathcal{O}}$, and let $R_{\Gamma}$ be the affine coordinate ring of the 0 -dimensional affine scheme represented by $\Gamma$. Then we have $\mathrm{HF}^{(n, \mu)} \succeq \mathrm{HF}_{R_{\Gamma}}^{a} \succeq \mathrm{HF}_{\mathcal{O}}^{a}$.

Proof. The generic affine Hilbert function for a scheme of length $\mu$ dominates the affine Hilbert function of $\mathbb{X}_{\Gamma}$, since its values exhibit the maximal possible growth.

On the other hand, the affine Hilbert function of $R_{\Gamma}$ dominates $\mathrm{HF}_{\mathcal{O}}^{a}$, since the terms of $\mathcal{O}$ are linearly independent in $R_{\Gamma}$.

Here is an easy example of an order ideal $\mathcal{O}$ such that there are 0-dimensional schemes represented by rational points in $\mathbb{B}_{\mathcal{O}}$ having different associated affine Hilbert functions.

Example 9.3. Let $P=K[x, y]$, and let $\mathcal{O}=\left\{1, y, y^{2}\right\} \subseteq \mathbb{T}^{2}$. Then we have $\mathrm{HF}_{\mathcal{O}}^{a}=(1,2,3,3, \ldots)$. Now let $I=\left\langle x, y^{3}\right\rangle$ and $J=\left\langle x-y^{2}, y^{3}\right\rangle$. The set $\left\{x, y^{3}\right\}$ is the reduced Lex-Gröbner basis of $I$, and the set $\left\{x-y^{2}, y^{3}\right\}$ is the reduced LexGröbner basis of $J$. Consequently, the rings $P / I$ and $P / J$ are coordinate rings of 0 -dimensional affine schemes represented by $K$-rational points in $\mathbb{B}_{\mathcal{O}}$. Then we have $\operatorname{HF}_{P / I}^{a}=(1,2,3,3, \ldots)$ and $\operatorname{HF}_{P / J}^{a}=(1,3,3, \ldots)$.

From the growth conditions for Hilbert functions in [18, Section 5.5, it follows that $(1,2,3,3, \ldots)$ and $(1,3,3, \ldots)$ are the only admissible affine Hilbert functions for a 0-dimensional scheme of length 3 in $\mathbb{A}_{K}^{2}$. Note that $\mathrm{HF}^{(2,3)}=\mathrm{HF}_{P / J}^{a} \succ \mathrm{HF}_{P / I}^{a}$.

In this example all admissible affine Hilbert functions are totally ordered with respect to $\succ$. The following example shows that this is not true in general.

Example 9.4. Consider all admissible affine Hilbert functions with $n=3$ and $\mu=11$. We have the relations $(1,4,10,11,11, \ldots) \succ(1,4,9,10,11,11, \ldots)$ and $(1,4,10,11,11, \ldots) \succ(1,4,8,11,11, \ldots)$, but the sequences $(1,4,9,10,11,11, \ldots)$ and $(1,4,8,11,11, \ldots)$ are not comparable with respect to $\succ$.

Our next goal is to describe the locus in $\mathbb{B}_{\mathcal{O}}$ of all 0-dimensional affine schemes whose affine Hilbert function is a given function $\mathcal{H}=\left(H_{0}, H_{1}, \ldots\right)$. For this purpose 
we first determine the equations defining a closed subscheme of $\mathbb{B}_{\mathcal{O}}$ such that its $K$-rational points represent those 0 -dimensional affine schemes whose affine Hilbert function is dominated by $\mathcal{H}$. As a preliminary step, we determine the closed subscheme of $\mathbb{B}_{\mathcal{O}}$ corresponding to all $K$-rational points whose associated affine Hilbert function satisfies $H_{k} \leq N$ for fixed given numbers $k \geq 0$ and $N>0$ as follows.

Algorithm 9.5. Let $\mathcal{O}=\left\{t_{1}, \ldots, t_{\mu}\right\}$ be an order ideal, let $k \geq 0$, and let $N \geq 1$. Consider the following sequence of instructions.

(1) Let $u_{1}, \ldots, u_{m} \in \mathbb{T}^{n}$ be the terms of degree $\leq k$.

(2) For $\ell=1, \ldots, m$, compute the matrix $\mathcal{U}_{\ell}=u_{\ell}\left(\mathcal{A}_{1}, \ldots, \mathcal{A}_{n}\right)$ in the ring $\operatorname{Mat}_{\mu}(K[C])$, where $\mathcal{A}_{1}, \ldots, \mathcal{A}_{n}$ are the generic multiplication matrices for $\mathcal{O}$.

(3) Form the matrix $\mathcal{M}$ in $\operatorname{Mat}_{\mu, m}(K[C])$ whose $\ell$-th column is the first column of $\mathcal{U}_{\ell}$ for $\ell=1, \ldots, m$.

(4) Compute the ideal $D$ of minors of size $N+1$ of $\mathcal{M}$ and return $J=I\left(\mathbb{B}_{\mathcal{O}}\right)+D$. This is an algorithm which computes an ideal $J$ in $K[C]$ such that $\mathcal{Z}(J)$ is the closed subscheme of $\mathbb{B}_{\mathcal{O}}$ corresponding to all $K$-rational points whose associated affine Hilbert function satisfies $H_{k} \leq N$.

Proof. The universal border basis family $U_{\mathcal{O}}$ is a free $B_{\mathcal{O}}$-module with basis $\mathcal{O}$, and for $\nu=1, \ldots, n$ the generic multiplication matrix $\mathcal{A}_{\nu}$ describes the multiplication by $x_{\nu}$ in this basis. Hence, for $\ell=1, \ldots, m$, the matrix $\mathcal{U}_{\ell}$ describes the multiplication by $u_{\ell}$ in the basis $\mathcal{O}$. Thus $t_{1}=1$ implies that the first column of $\mathcal{U}_{\ell}$ contains the coordinates of $u_{\ell}$ in the basis $\mathcal{O}$. Now the claim follows from the remark that we have $H_{k} \leq N$ for the affine Hilbert function associated to a $K$-rational point $\Gamma$ of $\mathbb{B}_{\mathcal{O}}$ if and only if at most $N$ terms in the set $\left\{u_{1}, \ldots, u_{m}\right\}$ are $K$-linearly independent modulo $\left\langle G_{\Gamma}\right\rangle$.

Let us check the results of this algorithm in a simple case.

Example 9.6. Let $\mathcal{O}$ be the order ideal $\mathcal{O}=\left\{1, x, y, x^{2}, x^{3}\right\}$ in $\mathbb{T}^{2}$. The border of $\mathcal{O}$ is $\partial \mathcal{O}=\left\{x y, y^{2}, x^{2} y, x^{4}, x^{3} y\right\}$. We have $\mu=5$, and the generic affine Hilbert function is $(1,3,5,5, \ldots)$.

When we use the algorithm to compute the closed subscheme of $\mathbb{B}_{\mathcal{O}}$ corresponding to $H_{2} \leq 4$, we obtain the ideal $I\left(\mathbb{B}_{\mathcal{O}}\right)+\left\langle c_{51}, c_{52}\right\rangle$. Notice that this ideal equals the ideal $I\left(\mathbb{B}_{\mathcal{O}}^{\text {df }}\right)$. This is due to the fact that the only possible affine Hilbert function of a $K$-rational point $\Gamma$ with $\mathrm{HF}_{R_{\Gamma}}(2) \leq 4$ is $(1,3,4,5,5, \ldots)$, and thus these are exactly the points for which $\mathcal{O}$ is a degree filtered $K$-basis of $R_{\Gamma}$.

However, if we use the algorithm to compute the closed subscheme of $\mathbb{B}_{\mathcal{O}}$ corresponding to $H_{2} \leq 3$, we obtain the unit ideal $\langle 1\rangle$. Although there exists an affine Hilbert function satisfying this constraint, namely $(1,2,3,4,5,5, \ldots)$, that affine Hilbert function is not attained by any 0 -dimensional affine scheme $\mathbb{X}$ whose vanishing ideal has an $\mathcal{O}$-border basis, because $\{1, x, y\} \subset \mathcal{O}$ implies that there is no linear polynomial in the vanishing ideal of $\mathbb{X}$. 
The above algorithm allows us to describe the following subscheme of $\mathbb{B}_{\mathcal{O}}$.

Definition 9.7. Let $\mathcal{O}=\left\{t_{1}, \ldots, t_{\mu}\right\}$ be an order ideal in $\mathbb{T}^{n}$, and assume that $\mathcal{H}=\left(H_{0}, H_{1}, \ldots\right)$ is an $(n, \mu)$-admissible affine Hilbert function. Then the closed subscheme of $\mathbb{B}_{\mathcal{O}}$ which is the closure of the set of $K$-rational points $\Gamma$ for which $\mathrm{HF}_{R_{\Gamma}}^{a}$ is dominated by $\mathcal{H}$ is called the $\overline{\mathcal{H}}$-subscheme of $\mathbb{B}_{\mathcal{O}}$ and is denoted by $\mathbb{B}_{\mathcal{O}}(\overline{\mathcal{H}})$.

An ideal defining $\mathbb{B}_{\mathcal{O}}(\overline{\mathcal{H}})$ can be calculated as follows.

\section{Algorithm 9.8. (Computing the $\overline{\mathcal{H}}$-Subscheme of $\mathbb{B}_{\mathcal{O}}$ )}

Let $\mathcal{O}=\left\{t_{1}, \ldots, t_{\mu}\right\}$ be an order ideal, and let $\mathcal{H}=\left(H_{0}, H_{1}, \ldots\right)$ be a sequence of non-negative integers which dominates $\mathrm{HF}_{\mathcal{O}}^{a}$. The following instructions define an algorithm which computes an ideal $I\left(\mathbb{B}_{\mathcal{O}}(\overline{\mathcal{H}})\right)$ in $K[C]$ that defines $\mathbb{B}_{\mathcal{O}}(\overline{\mathcal{H}})$.

(1) Let $\varrho=\min \left\{i \geq 0 \mid H_{i}=\mu\right\}$.

(2) For $i=1, \ldots, \varrho-1$, use Algorithm 9.5 to compute an ideal $J_{i}$ in $K[C]$ which defines the closed subscheme of $\mathbb{B}_{\mathcal{O}}$ whose $K$-rational points $\Gamma$ satisfy $\mathrm{HF}_{R_{\Gamma}}^{a}(i) \leq H_{i}$.

(3) Return the ideal $J_{1}+\cdots+J_{\varrho-1}$.

Proof. The finiteness of this algorithm is clear. The correctness follows from Algorithm 9.5, since an affine Hilbert function $\mathrm{HF}_{R_{\Gamma}}^{a}$ is dominated by $\mathcal{H}$ if and only if each of its values is less than or equal to the corresponding value of $\mathcal{H}$.

Let us apply this algorithm in the setting of Example 9.6 .

Example 9.9. In $\mathbb{T}^{2}$ we consider the order ideal $\mathcal{O}=\left\{1, x, y, x^{2}, x^{3}\right\}$, and we let $\mathcal{H}=(1,3,5,5, \ldots)$. When we compute the ideal $I\left(\mathbb{B}_{\mathcal{O}}(\overline{\mathcal{H}})\right)$ using the algorithm, we get $I\left(\mathbb{B}_{\mathcal{O}}\right)$. This means that we have $\mathbb{B}_{\mathcal{O}}(\overline{\mathcal{H}})=\mathbb{B}_{\mathcal{O}}$ here, in agreement with the observation that $\mathcal{H}$ is the generic affine Hilbert function for $n=2$ and $\mu=5$.

Definition 9.10. Let $\mathcal{O}=\left\{t_{1}, \ldots, t_{\mu}\right\} \in \mathbb{T}^{n}$ be an order ideal, and let $\mathcal{H}=$ $\left(H_{0}, H_{1}, \ldots\right)$ be an $(n, \mu)$-admissible affine Hilbert function. The open subscheme of $\mathbb{B}_{\mathcal{O}}(\overline{\mathcal{H}})$ which is the complement in $\mathbb{B}_{\mathcal{O}}(\overline{\mathcal{H}})$ of the closure of the set of all $K$-rational points $\Gamma$ for which the associated affine Hilbert function $\mathrm{HF}_{R_{\Gamma}}^{a}$ is strictly dominated by $\mathcal{H}$, is called the $\mathcal{H}$-subscheme of $\mathbb{B}_{\mathcal{O}}$ and is denoted by $\mathbb{B}_{\mathcal{O}}(\mathcal{H})$.

In other words, the $K$-rational points $\Gamma$ of $\mathbb{B}_{\mathcal{O}}(\mathcal{H})$ correspond to 0-dimensional rings $R_{\Gamma}$ whose affine Hilbert function is $\mathcal{H}$. The following algorithms allows us to clarify and compute the structure of $\mathbb{B}_{\mathcal{O}}(\mathcal{H})$.

Algorithm 9.11. (Computing the $\mathcal{H}$-Subscheme of $\mathbb{B}_{\mathcal{O}}$ ) Let $\mathcal{O}=\left\{t_{1}, \ldots, t_{\mu}\right\}$ be an order ideal, and let $\mathcal{H}=\left(H_{0}, H_{1}, \ldots\right)$ be a sequence of non-negative integers which dominates $\mathrm{HF}_{\mathcal{O}}^{a}$. The following instructions define an algorithm which computes an ideal $I\left(Z_{\mathcal{O}}(\overline{\mathcal{H}})\right)$ in $K[C]$ which contains $I\left(\mathbb{B}_{\mathcal{O}}(\overline{\mathcal{H}})\right)$ and defines a closed subscheme $Z_{\mathcal{O}}(\overline{\mathcal{H}})$ of $\mathbb{B}_{\mathcal{O}}(\overline{\mathcal{H}})$ with $\mathbb{B}_{\mathcal{O}}(\mathcal{H})=\mathbb{B}_{\mathcal{O}}(\overline{\mathcal{H}}) \backslash Z_{\mathcal{O}}(\overline{\mathcal{H}})$. 
(1) Let $\varrho=\min \left\{i \geq 0 \mid H_{i}=\mu\right\}$.

(2) For $i=1, \ldots, \varrho$, let $\mathcal{H}_{i}^{\prime}=\left(1, H_{1}, \ldots, H_{i-1}, H_{i}-1, H_{i+1}, H_{i+2}, \ldots\right)$.

(3) For $i=1, \ldots, \varrho$, check whether $H_{i}-1 \geq \mathrm{HF}_{\mathcal{O}}^{a}(i)$. If this is the case, use Algorithm 9.8 to compute the ideal $J_{i}$ defining the $\overline{\mathcal{H}_{i}^{\prime}}$-subscheme of $\mathbb{B}_{\mathcal{O}}$. Otherwise, let $J_{i}=\langle 1\rangle$.

(4) Compute the ideal $J_{1} \cap \cdots \cap J_{\varrho}$ and return it.

Proof. Note that, for a $K$-rational point $\Gamma \in \mathbb{B}_{\mathcal{O}}(\overline{\mathcal{H}})$, an affine Hilbert function $\mathrm{HF}_{R_{\Gamma}}^{a}$ is strictly smaller than $\mathcal{H}$ if and only if at least one of its values is strictly smaller than the corresponding value of $\mathcal{H}$. Since $\mathcal{H}$ dominates the affine Hilbert function of $\mathcal{O}$ and since $\mathcal{O}$ is $K$-linearly independent in $R_{\Gamma}$, there can be no such point $\Gamma$ if $H_{i}-1<\mathrm{HF}_{\mathcal{O}}^{a}(i)$. Thus we can drop the corresponding ideals $J_{i}$ from the intersection. Finally, we note that each ideal $J_{i}$ contains $I\left(\mathbb{B}_{\mathcal{O}}(\overline{\mathcal{H}})\right)$, and therefore also the resulting intersection ideal does so.

Let us apply this algorithm to the setting of Example 9.9

Example 9.12. As in Example 9.9, let $\mathcal{O}=\left\{1, x, y, x^{2}, x^{3}\right\} \subseteq \mathbb{T}^{2}$, and let $\mathcal{H}=(1,3,5,5, \ldots)$. We use Algorithm 9.11 to calculate the ideal $I\left(Z_{\mathcal{O}}(\overline{\mathcal{H}})\right)$ defining the complement of $\mathbb{B}_{\mathcal{O}}(\mathcal{H})$ in $\mathbb{B}_{\mathcal{O}}(\overline{\mathcal{H}})$. Since $3=\mathrm{HF}_{\mathcal{O}}^{a}(1)$, we do not need to compute $J_{1}$. In order to find $J_{2}$, it suffices to take $I\left(\mathbb{B}_{\mathcal{O}}\right)$ and to add the ideal computed by Algorithm 9.5 for $i=2$ and $N=4$. The result is $I\left(\mathbb{B}_{\mathcal{O}}\right)+\left\langle c_{51}, c_{52}\right\rangle$, as noted in Example 9.6. Altogether, we obtain $I\left(Z_{\mathcal{O}}(\overline{\mathcal{H}})\right)=I\left(\mathbb{B}_{\mathcal{O}}\right)+\left\langle c_{51}, c_{52}\right\rangle$.

Notice that the algorithm correctly returns $I\left(\mathbb{B}_{\mathcal{O}}(\overline{\mathcal{H}})\right)$ if the function $\mathcal{H}$ is not $(n, \mu)$-admissible. The following example illustrates this behaviour.

Example 9.13. In $\mathbb{T}^{2}$, consider the order ideal $\mathcal{O}=\left\{1, x, x^{2}, x^{3}\right\}$. The sequence $\mathcal{H}=(1,2,4,4, \ldots)$ dominates the affine Hilbert function $\operatorname{HF}_{\mathcal{O}}^{a}=(1,2,3,4,4, \ldots)$ of $\mathcal{O}$. Algorithm 9.11 yields the ideals $J_{1}=\langle 1\rangle$ and $J_{2}=I\left(\mathbb{B}_{\mathcal{O}}\left(\overline{\mathcal{H}_{2}^{\prime}}\right)\right)=I\left(\mathbb{B}_{\mathcal{O}}(\overline{\mathcal{H}})\right)$, and hence returns $I\left(\mathbb{B}_{\mathcal{O}}(\overline{\mathcal{H}})\right)$. Thus it follows that $\mathbb{B}_{\mathcal{O}}(\mathcal{H})=\mathbb{B}_{\mathcal{O}}(\overline{\mathcal{H}}) \backslash \mathbb{B}_{\mathcal{O}}(\overline{\mathcal{H}})=\emptyset$. This is in agreement with the fact that $\mathcal{H}$ is not $(2,4)$-admissible.

Corollary 9.14. Let $\mathcal{O}=\left\{t_{1}, \ldots, t_{\mu}\right\}$ be an order ideal in $\mathbb{T}^{n}$.

(a) Let $\mathcal{H}$ be an $(n, \mu)$-admissible affine Hilbert function with $\mathrm{HF}^{(n, \mu)} \succeq \mathcal{H} \succeq \mathrm{HF}_{\mathcal{O}}^{a}$. Then $\mathbb{B}_{\mathcal{O}}(\mathcal{H})$ is an open subscheme of $\mathbb{B}_{\mathcal{O}}(\overline{\mathcal{H}})$ and a locally closed subscheme of $\mathbb{B}_{\mathcal{O}}$. It is called the $\mathcal{H}$-Hilbert stratum of $\mathbb{B}_{\mathcal{O}}$.

(b) Let $\mathbb{H}_{\mathcal{O}}$ be the set of all $(n, \mu)$-admissible affine Hilbert functions $\mathcal{H}$ with $\mathrm{HF}^{(n, \mu)} \succeq \mathcal{H} \succeq \mathrm{HF}_{\mathcal{O}}^{a}$. Then we have a disjoint union $\mathbb{B}_{\mathcal{O}}=\bigcup_{\mathcal{H} \in \mathbb{H}_{\mathcal{O}}} \mathbb{B}_{\mathcal{O}}(\mathcal{H})$. This is called the Hilbert stratification of $\mathbb{B}_{\mathcal{O}}$.

Proof. The claims in (a) follow from Algorithm 9.11 and Algorithm 9.8 Claim (b) follows from Proposition 9.2 
The order ideals making up the Hilbert stratification of $\mathbb{B}_{\mathcal{O}}$ can be determined as follows.

Remark 9.15. Let $\mathcal{O}=\left\{t_{1}, \ldots, t_{\mu}\right\}$ be an order ideal in $\mathbb{T}^{n}$. Consider the recursively defined function $\operatorname{AllStrata}(\mathcal{O}, \mathcal{H})$ defined by the following steps.

(1) Let $S$ be the set consisting of $\mathcal{H}$.

(2) Let $\mathcal{H}=\left(H_{0}, H_{1}, \ldots\right)$, and let $\varrho=\min \left\{i \geq 0 \mid H_{i}=\mu\right\}$.

(3) For $i=1, \ldots, \varrho$, check whether $H_{i}-1 \geq \operatorname{HF}_{\mathcal{O}}^{a}(i)$. If this is the case, compute $\operatorname{AllStrata}\left(\mathcal{O}, \mathcal{H}^{\prime}\right)$, where $\mathcal{H}^{\prime}=\left(H_{0}, \ldots, H_{i-1}, H_{i}-1, H_{i+1}, \ldots\right)$, append it to $S$, and remove doubles in $S$.

(4) If $\mathcal{H}=\mathrm{HF}_{\mathcal{O}}^{a}$ then return $S$ and stop.

Let $S$ be the result of calling $\operatorname{All} \operatorname{Strata}\left(\mathcal{O}, \operatorname{HF}^{(n, \mu)}\right)$. For every sequence $\mathcal{H}$ in $S$, use [18, Theorem 5.5.32.a, to check whether $\mathcal{H}$ is admissible. If this is not the case, remove $\mathcal{H}$ from $S$. The resulting set $S$ is the set of all $(n, \mu)$-admissible affine Hilbert functions with $\mathrm{HF}^{(n, \mu)} \succeq \mathcal{H} \succeq \mathrm{HF}_{\mathcal{O}}^{a}$.

\section{An Open Covering of the Hilbert Stratum}

In several of the algorithms in the next sections it will be necessary not only to fix the Hilbert function of $R=P / I$, but also a degree filtered $K$-basis of $R$.

As above, let $K$ be a field, and let $\mathcal{O}=\left\{t_{1}, \ldots, t_{\mu}\right\}$ be an order ideal with border $\partial \mathcal{O}=\left\{b_{1}, \ldots, b_{\nu}\right\}$. In this setting, we introduce the following subschemes of $\mathbb{B}_{\mathcal{O}}$.

Definition 10.1. Let $\mathcal{O}=\left\{t_{1}, \ldots, t_{\mu}\right\}$ be an order ideal.

(a) Let $\mathcal{O}^{\prime}=\left\{t_{1}^{\prime}, \ldots, t_{\mu}^{\prime}\right\}$ be a further order ideal containing $\mu$ terms. We say that $\mathcal{O}^{\prime}$ is a degree filtered order ideal for $\mathcal{O}$ if there exists an ideal $I \subseteq P$ which has an $\mathcal{O}$-border basis and for which $\mathcal{O}^{\prime}$ is a degree filtered $K$-basis of $P / I$.

(b) Let $\mathcal{O}^{\prime}$ be a degree filtered order ideal for $\mathcal{O}$, and let $\mathcal{H}=\left(H_{0}, H_{1}, \ldots\right)$ be the affine Hilbert function of $\mathcal{O}^{\prime}$. The open subscheme of $\mathbb{B}_{\mathcal{O}}(\mathcal{H})$ whose $K$ rational points represent the 0 -dimensional affines schemes $\mathbb{X}$ such that $\mathcal{O}^{\prime}$ is a degree filtered $K$-basis of $R_{\mathbb{X}}$ (and hence $\mathcal{H}=\mathrm{HF}_{\mathbb{X}}^{a}$ ) is called the $\mathcal{O}^{\prime}$-DFB subscheme and is denoted by $\mathbb{B}_{\mathcal{O}}^{\mathrm{dfb}}\left(\mathcal{O}^{\prime}\right)$.

The next algorithm shows that $\mathbb{B}_{\mathcal{O}}^{\mathrm{dfb}}\left(\mathcal{O}^{\prime}\right)$ is in fact an open subscheme of $\mathbb{B}_{\mathcal{O}}(\overline{\mathcal{H}})$ which is contained in $\mathbb{B}_{\mathcal{O}}(\mathcal{H})$.

\section{Algorithm 10.2. (Computing a DFB Subscheme)}

Let $\mathcal{O}=\left\{t_{1}, \ldots, t_{\mu}\right\}$ be an order ideal in $\mathbb{T}^{n}$, let $\mathcal{H}$ be an $(n, \mu)$-admissible Hilbert function which dominates $\mathrm{HF}_{\mathcal{O}}^{a}$, and let $\mathcal{O}^{\prime}=\left\{t_{1}^{\prime}, \ldots, t_{\mu}^{\prime}\right\}$ be an order ideal such that $\mathrm{HF}_{\mathcal{O}^{\prime}}^{a}=\mathcal{H}$. Consider the following instructions.

(1) Using Algorithm 9.8, compute an ideal $I\left(\mathbb{B}_{\mathcal{O}}(\overline{\mathcal{H}})\right)$ which defines $\mathbb{B}_{\mathcal{O}}(\overline{\mathcal{H}})$. 
(2) Using Algorithm 9.11, applied to $\mathcal{H}$, compute an ideal $I\left(Z_{\mathcal{O}}(\overline{\mathcal{H}})\right)$ which defines the closed subscheme $Z_{\mathcal{O}}(\overline{\mathcal{H}})$ of $\mathbb{B}_{\mathcal{O}}(\overline{\mathcal{H}})$.

(3) For $i=1, \ldots, \mu$, calculate the matrix $t_{i}^{\prime}\left(\mathcal{A}_{1}, \ldots \mathcal{A}_{n}\right)$, where $\mathcal{A}_{1}, \ldots, \mathcal{A}_{n}$ are the generic multiplication matrices for $\mathcal{O}$.

(4) Form the matrix $T^{\prime}$ in $\operatorname{Mat}_{\mu}(K[C])$ consisting of the first columns of the matrices $t_{i}^{\prime}\left(\mathcal{A}_{1}, \ldots, \mathcal{A}_{n}\right)$ for $i=1, \ldots, \mu$.

(5) Return the triple $\left(I\left(\mathbb{B}_{\mathcal{O}}(\overline{\mathcal{H}})\right), I\left(Z_{\mathcal{O}}(\overline{\mathcal{H}})\right), \operatorname{det}\left(T^{\prime}\right)\right)$.

This is an algorithm which computes a triple $\left(I\left(\mathbb{B}_{\mathcal{O}}(\overline{\mathcal{H}})\right), I\left(Z_{\mathcal{O}}(\overline{\mathcal{H}})\right), \operatorname{det}\left(T^{\prime}\right)\right)$ such that, if we let $I_{\mathcal{O O}^{\prime}}^{\mathrm{dfb}}=I\left(Z_{\mathcal{O}}(\overline{\mathcal{H}})\right) \cap\left\langle\operatorname{det}\left(T^{\prime}\right)\right\rangle$, then $J_{\mathcal{O}^{\prime}}=I_{\mathcal{O O}^{\prime}}^{\mathrm{dfb}}+I\left(\mathbb{B}_{\mathcal{O}}(\overline{\mathcal{H}})\right)$ satisfies $\mathbb{B}_{\mathcal{O}}^{\mathrm{dfb}}\left(\mathcal{O}^{\prime}\right)=\mathbb{B}_{\mathcal{O}}(\overline{\mathcal{H}}) \backslash \mathcal{Z}\left(J_{\mathcal{O}^{\prime}}\right)=\mathbb{B}_{\mathcal{O}}(\mathcal{H}) \backslash \mathcal{Z}\left(\operatorname{det}\left(T^{\prime}\right)\right)$.

Proof. Since $\mathcal{H}=\mathrm{HF}_{\mathcal{O}^{\prime}}^{a}$, the set $\mathcal{O}^{\prime}$ is a degree filtered $K$-basis of $R_{\mathbb{X}_{\Gamma}}$ for some closed point $\Gamma$ of $\mathbb{B}_{\mathcal{O}}$ representing a 0 -dimensional affine scheme $\mathbb{X}_{\Gamma}$ if and only if it is a $K$-basis.

For $i=1, \ldots, \mu$, the first column of the matrix $t_{i}^{\prime}\left(\mathcal{A}_{1}, \ldots, \mathcal{A}_{n}\right)$ contains the coordinates of $t_{i}^{\prime}$ in the $K$-basis $\mathcal{O}$ of $U_{\mathcal{O}}$, because this matrix is the multiplication matrix of $t_{i}^{\prime}$ and we assumed $t_{1}=1$. Hence, at a $K$-rational point $\Gamma=\left(\gamma_{i j}\right)$ of $\mathbb{B}_{\mathcal{O}}$, we have $\operatorname{det}\left(T^{\prime}\right)\left(\gamma_{i j}\right) \neq 0$ if and only if $\mathcal{O}^{\prime}$ is a $K$-basis of $R_{\Gamma}$. Since the set $\mathbb{B}_{\mathcal{O}}(\mathcal{H})$ is the complement of $\mathcal{Z}\left(I\left(Z_{\mathcal{O}}(\overline{\mathcal{H}})\right)\right)$ in $\mathbb{B}_{\mathcal{O}}(\overline{\mathcal{H}})$, the proof is complete.

The sets $\mathbb{B}_{\mathcal{O}}^{\mathrm{dfb}}\left(\mathcal{O}^{\prime}\right)$ form an open covering of $\mathbb{B}_{\mathcal{O}}(\mathcal{H})$, as the next corollary shows.

Corollary 10.3. In the setting of the algorithm, let $\mathcal{O}_{1}^{\prime}, \ldots, \mathcal{O}_{\ell}^{\prime}$ be the order ideals in $\mathbb{T}^{n}$ with affine Hilbert function $\mathcal{H}$.

(a) For $i=1, \ldots, \ell$, the set $\mathbb{B}_{\mathcal{O}}^{\mathrm{dfb}}\left(\mathcal{O}_{i}^{\prime}\right)$ is an open subscheme of $\mathbb{B}_{\mathcal{O}}(\overline{\mathcal{H}})$ which is contained in $\mathbb{B}_{\mathcal{O}}(\mathcal{H})$.

(b) The subschemes $\mathbb{B}_{\mathcal{O}}^{\mathrm{dfb}}\left(\mathcal{O}_{1}^{\prime}\right), \ldots, \mathbb{B}_{\mathcal{O}}^{\mathrm{dfb}}\left(\mathcal{O}_{\ell}^{\prime}\right)$ form an open covering of $\mathbb{B}_{\mathcal{O}}(\mathcal{H})$.

Proof. To show (a), we note that

$$
J_{\mathcal{O}_{i}}+I\left(\mathbb{B}_{\mathcal{O}}(\overline{\mathcal{H}})\right)=\left(I\left(Z_{\mathcal{O}}(\overline{\mathcal{H}})\right) \cap\left\langle\operatorname{det}\left(T_{i}^{\prime}\right)\right\rangle\right)+I\left(\mathbb{B}_{\mathcal{O}}(\overline{\mathcal{H}})\right) \subseteq I\left(Z_{\mathcal{O}}(\overline{\mathcal{H}})\right)
$$

for $i=1, \ldots, \ell$, and that we have $\mathbb{B}_{\mathcal{O}}(\mathcal{H})=\mathbb{B}_{\mathcal{O}}(\overline{\mathcal{H}}) \backslash \mathcal{Z}\left(I\left(Z_{\mathcal{O}}(\overline{\mathcal{H}})\right)\right)$.

Now we prove (b). For every $K$-rational point $\Gamma$ of $\mathbb{B}_{\mathcal{O}}(\mathcal{H})$, we consider the order ideal $\mathcal{O}_{\sigma}\left(I_{\Gamma}\right)=\mathbb{T}^{n} \backslash \operatorname{LT}_{\sigma}\left(I_{\Gamma}\right)$ for some degree compatible term ordering $\sigma$. Then the point $\Gamma$ is contained in $\mathbb{B}_{\mathcal{O}}^{\mathrm{dfb}}\left(\mathcal{O}_{\sigma}\left(I_{\Gamma}\right)\right)$, and the claim follows.

To complement this discussion, we apply Algorithm 10.2 to the setting of Example 9.6

Example 10.4. Let $\mathcal{O}$ be the order ideal $\mathcal{O}=\left\{1, x, y, x^{2}, x^{3}\right\}$ in $\mathbb{T}^{2}$, and let $\mathcal{H}=$ $(1,3,5,5, \ldots)$. In order to cover $\mathbb{B}_{\mathcal{O}}(\mathcal{H})$ with DFB-subschemes, we have to construct all order ideals with affine Hilbert function $\mathcal{H}$. They are $\mathcal{O}_{1}^{\prime}=\left\{1, x, y, x^{2}, x y\right\}$, $\mathcal{O}_{2}^{\prime}=\left\{1, x, y, x^{2}, y^{2}\right\}$, and $\mathcal{O}_{3}^{\prime}=\left\{1, x, y, x y, y^{2}\right\}$. 
(a) When we apply Algorithm 10.2 to $\mathcal{O}_{1}^{\prime}$, we get $J_{\mathcal{O}_{1}^{\prime}}=\left\langle c_{51}\right\rangle+I\left(\mathbb{B}_{\mathcal{O}}(\overline{\mathcal{H}})\right)$. This is consistent with the observation that we can exchange the element $x^{3}$ of $\mathcal{O}$ with the element $x y$ of $\mathcal{O}_{1}^{\prime}$ in the basis of a ring $R_{\mathbb{X}_{\Gamma}}$ if an only if the entry $c_{51}$ in $\Gamma$ is non-zero.

(b) An application of Algorithm 10.2 to $\mathcal{O}_{2}^{\prime}$ yields $J_{\mathcal{O}_{2}^{\prime}}=\left\langle c_{52}\right\rangle+I\left(\mathbb{B}_{\mathcal{O}}(\overline{\mathcal{H}})\right)$.

(c) By applying Algorithm 10.2 to $\mathcal{O}_{3}^{\prime}$, we get $J_{\mathcal{O}_{3}^{\prime}}=\left\langle c_{42} c_{51}-c_{41} c_{52}\right\rangle+I\left(\mathbb{B}_{\mathcal{O}}(\overline{\mathcal{H}})\right)$.

Our next task is the following. Suppose we are given a function $\operatorname{Locus}\left(\mathcal{O}^{\prime}\right)$ which returns an ideal $I_{\mathcal{O}^{\prime}}$ in $K\left[C^{\prime}\right]$ such that $\mathbb{B}_{\mathcal{O}^{\prime}}^{\text {df }} \backslash \mathcal{Z}\left(I_{\mathcal{O}^{\prime}}\right)$ contains exactly the $K$-rational points which represent schemes having a degree filtered $\mathcal{O}^{\prime}$-border basis and a certain property $\mathcal{P}$. After covering $\mathbb{B}_{\mathcal{O}}(\mathcal{H})$ with finitely many open subsets $\mathbb{B}_{\mathcal{O}}^{\text {dfb }}\left(\mathcal{O}^{\prime}\right)$, we want to compute an ideal $J$ in $K[C]$ such that $\mathbb{B}_{\mathcal{O}}(\mathcal{H}) \backslash \mathcal{Z}(J)$ contains exactly the $K$-rational points of $\mathbb{B}_{\mathcal{O}}(\mathcal{H})$ which represent the 0 -dimensional schemes with Hilbert function $\mathcal{H}$ and property $\mathcal{P}$.

The first step is to construct some base change formulas over certain open subsets of $\mathbb{B}_{\mathcal{O}}$.

Proposition 10.5. Let $\mathcal{O}=\left\{t_{1}, \ldots, t_{\mu}\right\}$ and $\mathcal{O}^{\prime}=\left\{t_{1}^{\prime}, \ldots, t_{\mu}^{\prime}\right\}$ be two order ideals such that there is a 0-dimensional subscheme $\mathbb{X}$ of $\mathbb{A}_{K}^{n}$ which has both an $\mathcal{O}$-border basis and an $\mathcal{O}^{\prime}$-border basis.

(a) For $i \in\{1, \ldots, \mu\}$, let $T_{i}^{\prime}$ be the first column of $t_{i}^{\prime}\left(\mathcal{A}_{1}, \ldots, \mathcal{A}_{n}\right)$, where $\mathcal{A}_{1}, \ldots, \mathcal{A}_{n}$ are the generic multiplication matrices with respect to $\mathcal{O}$. Let $T^{\prime} \in \operatorname{Mat}_{\mu}(K[C])$ be the matrix with columns $T_{1}^{\prime}, \ldots, T_{\mu}^{\prime}$, and let $\delta=\operatorname{det}\left(T^{\prime}\right)$. Then the open subset $D(\delta)$ of $\mathbb{B}_{\mathcal{O}}$ parametrizes all $K$-rational points of $\mathbb{B}_{\mathcal{O}}$ which represent schemes having an $\mathcal{O}^{\prime}$-border basis.

(b) Let $G=\left\{g_{1}, \ldots, g_{\nu}\right\}$ be the generic $\mathcal{O}$-border prebasis, and let $U_{\mathcal{O}}=$ $\mathbb{B}_{\mathcal{O}}\left[x_{1}, \ldots, x_{n}\right] /\langle G\rangle$ be the universal $\mathcal{O}$-border basis family. Then both $\mathcal{O}$ and $\mathcal{O}^{\prime}$ are $K[C]_{\delta}$-bases of $\left(U_{\mathcal{O}}\right)_{\delta}$, and if we consider $\mathcal{O}$ and $\mathcal{O}^{\prime}$ as row vectors, we have $\mathcal{O}^{\prime}=\mathcal{O} \cdot T^{\prime}$.

(c) In the setting of (b), let $f \in P$. Then the matrices of the multiplication by $f$ with respect to the $K[C]$-bases $\mathcal{O}$ and $\mathcal{O}^{\prime}$ on $\left(U_{\mathcal{O}}\right)_{\delta}$ satisfy the formula

$$
\mathcal{A}_{f}^{\prime}=\frac{1}{\delta} \operatorname{adj}\left(T^{\prime}\right) \cdot \mathcal{A}_{f} \cdot T^{\prime}
$$

Proof. To prove (a) and (b), we note that the matrix $t_{i}^{\prime}\left(\mathcal{A}_{1}, \ldots, \mathcal{A}_{n}\right)$ is the multiplication matrix of $t_{i}^{\prime}$ in the basis $\mathcal{O}$ of the universal family. By the ordering of the terms, we have $t_{1}=t_{1}^{\prime}=1$. Hence the first column of $t_{i}^{\prime}\left(\mathcal{A}_{1}, \ldots, \mathcal{A}_{n}\right)$ contains the tuple of coordinates for $t_{i}^{\prime}$ in the basis $\mathcal{O}$. Consequently, the tuple $\left(t_{1}^{\prime}, \ldots, t_{\mu}^{\prime}\right)$ is a $K[C]$-basis of $U_{\mathcal{O}}$ at all points where $\operatorname{det}\left(T^{\prime}\right) \neq 0$, and this is precisely the set $D(\delta)$. Moreover, the transformation matrix from the basis $\mathcal{O}$ to the basis $\mathcal{O}^{\prime}$ is given by $T^{\prime}$ over the set $D(\delta)$.

Claim (c) follows from the base change formula for linear maps and the observation that $\left(T^{\prime}\right)^{-1}=\frac{1}{\delta} \operatorname{adj}\left(T^{\prime}\right)$.

The next proposition provides a way to relate the coordinate systems $C=\left\{c_{i j}\right\}$ 
of $\mathbb{B}_{\mathcal{O}}$ and $C^{\prime}=\left\{c_{i j}^{\prime}\right\}$ of $\mathbb{B}_{\mathcal{O}^{\prime}}$ when an open subset of the latter is mapped to $\mathbb{B}_{\mathcal{O}}^{\mathrm{dfb}}\left(\mathcal{O}^{\prime}\right)$.

Proposition 10.6. Let $\mathcal{O}=\left\{t_{1}, \ldots, t_{\mu}\right\}$ and $\mathcal{O}^{\prime}=\left\{t_{1}^{\prime}, \ldots, t_{\mu}^{\prime}\right\}$ be two order ideals such that there exists a 0-dimensional subscheme $\mathbb{X}$ of $\mathbb{A}_{K}^{n}$ which is represented both by a point in $\mathbb{B}_{\mathcal{O}}$ and by a point in $\mathbb{B}_{\mathcal{O}^{\prime}}$. Let $C^{\prime}=\left\{c_{i j}^{\prime}\right\}$ be the set of indeterminates such that $\mathbb{B}_{\mathcal{O}^{\prime}}=K\left[C^{\prime}\right] / I\left(\mathbb{B}_{\mathcal{O}^{\prime}}\right)$, and let $\partial \mathcal{O}^{\prime}=\left\{b_{1}^{\prime}, \ldots, b_{\ell}^{\prime}\right\}$.

For $j=1, \ldots, \ell$, let $B_{j}^{\prime}=\left(b_{1 j}^{\prime}, \ldots, b_{\mu j}^{\prime}\right)^{\text {tr }}$ be the first column of $b_{j}^{\prime}\left(\mathcal{A}_{1}, \ldots, \mathcal{A}_{n}\right)$. Then the representation of $c_{i j}^{\prime}$ in the coordinate system $C$ is given by the $i$-th entry of $\left(T^{\prime}\right)^{-1} B_{j}^{\prime}$ which is of the form $\frac{1}{\delta} p_{i j}$ with $p_{i j} \in K[C]$ for $i=1, \ldots, \mu$ and $j=1, \ldots, \ell$.

Proof. For $j=1, \ldots, \ell$, consider the coordinate tuple of $b_{j}^{\prime}$ in the basis $\mathcal{O}^{\prime}$ of $\left(U_{\mathcal{O}}\right)_{\delta}$. One one hand, it is $\left(c_{1 j}^{\prime}, \ldots, c_{\mu j}^{\prime}\right)^{\text {tr }}$. On the other hand, as shown in the proof of the preceding proposition, the coordinate tuple of $b_{j}^{\prime}$ in the basis $\mathcal{O}$ is $B_{j}^{\prime}$. Consequently, we get $b_{j}^{\prime}=\mathcal{O} \cdot B_{j}^{\prime}=\mathcal{O}^{\prime} \cdot\left(T^{\prime}\right)^{-1} B_{j}^{\prime}$. By comparing the coefficients of $b_{j}^{\prime}$ in the basis $\mathcal{O}^{\prime}$, we see that $c_{i j}^{\prime}$ corresponds to the $i$-th entry of $\left(T^{\prime}\right)^{-1} B_{j}^{\prime}$. Using $\left(T^{\prime}\right)^{-1}=\frac{1}{\delta} \operatorname{adj}\left(T^{\prime}\right)$, the claim follows.

Next we tackle the main task described above: how to transform the output of the function $\operatorname{Locus}\left(\mathcal{O}^{\prime}\right)$ for various order ideals $\mathcal{O}^{\prime}$ to a single ideal defining the desired locus in $\mathbb{B}_{\mathcal{O}}(\mathcal{H})$.

\section{Algorithm 10.7. (Combining Loci in a Hilbert Stratum)}

Let $\mathcal{O}=\left\{t_{1}, \ldots, t_{\mu}\right\}$ be an order ideal in $\mathbb{T}^{n}$, and let $\mathcal{H}$ be an $(n, \mu)$-admissible Hilbert function which dominates $\mathrm{HF}_{\mathcal{O}}^{a}$. Suppose that there exists a function Locus $\left(\mathcal{O}^{\prime}\right)$ which returns, for every order ideal $\mathcal{O}^{\prime}$ with $\mathrm{HF}_{\mathcal{O}^{\prime}}^{a}=\mathcal{H}$, an ideal $J_{\mathcal{O}^{\prime}}$ in $K\left[C^{\prime}\right]$ such that $\mathbb{B}_{\mathcal{O}^{\prime}}^{\mathrm{df}} \backslash \mathcal{Z}\left(J_{\mathcal{O}^{\prime}}\right)$ contains exactly the $K$-rational points which represent schemes having a degree filtered $\mathcal{O}^{\prime}$-border basis and a certain property $\mathcal{P}$. Consider the following instructions.

(1) Using Algorithms 9.8 and 9.11, compute the ideals $I\left(\mathbb{B}_{\mathcal{O}}(\overline{\mathcal{H}})\right)$ and $I\left(Z_{\mathcal{O}}(\overline{\mathcal{H}})\right)$ in $K[C]$.

(2) Find the set $\left\{\mathcal{O}_{1}^{\prime}, \ldots, \mathcal{O}_{\ell}^{\prime}\right\}$ of all order ideals $\mathcal{O}_{i}^{\prime}$ such that $\mathcal{H}=\mathrm{HF}_{\mathcal{O}_{i}^{\prime}}^{a}$.

(3) For $i=1, \ldots, \ell$, perform the following Steps (4) - (8).

(4) Using $\operatorname{Locus}\left(\mathcal{O}_{i}^{\prime}\right)$, calculate the ideal $J_{\mathcal{O}_{i}^{\prime}}$ in $K\left[C_{i}^{\prime}\right]$, where $C_{i}^{\prime}$ is the set of indetermintes such that $B_{\mathcal{O}_{i}^{\prime}}=K\left[C_{i}^{\prime}\right] / I\left(\mathbb{B}_{\mathcal{O}_{i}^{\prime}}\right)$. Let $J_{\mathcal{O}_{i}^{\prime}}=\left\langle a_{i 1}, \ldots, a_{i r_{i}}\right\rangle$.

(5) Use Proposition 10.5. a to find the polynomial $\delta_{i} \in K[C]$ such that $D\left(\delta_{i}\right)$ is the open subset of $\mathbb{B}_{\mathcal{O}}(\overline{\mathcal{H}})$ whose $K$-rational points represent schemes having a degree filtered $\mathcal{O}^{\prime}$-border basis.

(6) Using Proposition 10.6, compute polynomials $p_{\kappa \lambda} \in K[C]$ for $\kappa=1, \ldots, \mu$ and $\lambda=1, \ldots, \# \partial \mathcal{O}_{i}^{\prime}$ such that $c_{\kappa \lambda}^{\prime}$ corresponds to $\frac{1}{\delta_{i}} p_{\kappa \lambda}$.

(7) Let $z$ be a new indeterminate. For $j=1, \ldots, r_{i}$, compute the homogenization $a_{i j}^{*} \in K\left[C_{i}^{\prime}\right][z]$ of $a_{i j}$ with respect to $z$. Then let $\hat{a}_{i j} \in K[C]$ be the result of substituting $c_{\kappa \lambda}^{\prime} \mapsto p_{\kappa \lambda}$ and $z \mapsto \delta_{i}$ in $a_{i j}^{*}$. Let $J_{i}=\left\langle\hat{a}_{i 1}, \ldots, \hat{a}_{i r_{i}}\right\rangle$. 
(8) Compute $I_{\mathcal{O O}_{i}^{\prime}}^{\mathrm{dfb}}=I\left(Z_{\mathcal{O}}(\overline{\mathcal{H}})\right) \cap\left\langle\delta_{i}\right\rangle$.

(9) Return the list of triples $\left(\mathcal{O}_{i}^{\prime}, I_{\mathcal{O O}_{i}^{\prime}}^{\mathrm{dfb}}, J_{i}\right)$ where $i=1, \ldots, \ell$.

This is an algorithm which computes a list of triples $\left(\mathcal{O}_{i}^{\prime}, I_{\mathcal{O O}_{i}^{\prime}}^{\mathrm{dfb}}, J_{i}\right)$ of ideals such that, for the ideal

$$
J=\sum_{i=1}^{\ell}\left(\left(I_{\mathcal{O O}}^{\mathrm{dfb}}+I\left(\mathbb{B}_{\mathcal{O}}(\overline{\mathcal{H}})\right)\right) \cap\left(J_{i}+I\left(\mathbb{B}_{\mathcal{O}}(\overline{\mathcal{H}})\right)\right)\right)
$$

the set $\mathbb{B}_{\mathcal{O}}(\overline{\mathcal{H}}) \backslash \mathcal{Z}(J)$ contains precisely the $K$-rational points of $\mathbb{B}_{\mathcal{O}}(\mathcal{H})$ which represent schemes having property $\mathcal{P}$.

Proof. Finiteness of the algorithm is clear. Let us start the correctness proof by looking at the formula for the resulting ideal $J$. Clearly, if a $K$-rational point $\Gamma$ of $\mathbb{B}_{\mathcal{O}}(\overline{\mathcal{H}})$ represents a scheme having property $\mathcal{P}$, it is in the set of all $K$-rational points of $\mathbb{B}_{\mathcal{O}}^{\mathrm{df}}\left(\mathcal{O}^{\prime}\right)$ with this property for every order ideal $\mathcal{O}^{\prime}$ for which $\mathcal{O}^{\prime}$ is a degree filtered $K$-basis of $R_{\Gamma}$. Hence $J$ is the sum of the ideals defining the corresponding complements in $\mathbb{B}_{\mathcal{O}}^{\mathrm{df}}\left(\mathcal{O}^{\prime}\right)$. In view of Algorithm 10.2, it remains to show that $J_{i}+I\left(\mathbb{B}_{\mathcal{O}}(\overline{\mathcal{H}})\right)$ defines the correct subset of $\mathbb{B}_{\mathcal{O}}(\overline{\mathcal{H}})$.

By the hypothesis, the ideal $J_{\mathcal{O}_{i}^{\prime}}$ defines the correct locus in $\mathbb{B}_{\mathcal{O}_{i}^{\prime}}^{\text {df }}$. In Step (6) we apply the transformation rule for the elements $c_{i j}^{\prime}$ given in Proposition 10.5. However, when we transform the polynomials $a_{i j}$, we are always working in the localization $K[C]_{\delta_{i}}$. Hence we may multiply the resulting rational functions by a power of $\delta_{i}$ without changing the zero locus. The minimal power of $\delta_{i}$ with which $\left.a_{i j}\right|_{c_{\kappa \lambda}^{\prime} \mapsto p_{\kappa \lambda} / \delta_{i}}$ has to be multiplied to make it a polynomial is computed in Step (7) using the homogenization of $a_{i j}$. Altogether, the ideal $J_{i}=\left\langle\hat{a}_{i 1}, \ldots, \hat{a}_{i r}\right\rangle$ defines the same zero locus in $D\left(\delta_{i}\right) \subseteq \mathbb{B}_{\mathcal{O}}(\overline{\mathcal{H}})$ as the image of $J_{i}$ under $c_{\kappa \lambda}^{\prime} \mapsto \frac{1}{\delta_{i}} p_{\kappa \lambda}$, and the correctness proof of the algorithm is complete.

Recall that the vanishing ideals we compute need not be radical ideals. Using this freedom, the recombination of the different ideals $J_{i}$ in the preceding algorithm may profit from the following observation.

Lemma 10.8. Given a ring $S$ and ideals $I_{1}, \ldots, I_{r}, J$ in $S$, we have

$$
\operatorname{Rad}\left(\prod_{i=1}^{r} I_{i}+J\right)=\operatorname{Rad}\left(\bigcap_{i=1}^{r} I_{i}+J\right)=\operatorname{Rad}\left(\bigcap_{i=1}^{r}\left(I_{i}+J\right)\right)
$$

Proof. The inclusions " $\subseteq$ " are clear. Therefore it suffices to show $\bigcap_{i=1}^{r}\left(I_{i}+J\right) \subseteq$ $\operatorname{Rad}\left(\prod_{i=1}^{r} I_{i}+J\right)$. Let $f=a_{1}+b_{1}=\cdots=a_{r}+b_{r}$ with $a_{i} \in I_{i}$ and $b_{i} \in J$ for $i=1, \ldots, r$. Then we have $f^{r}=\prod_{i=1}^{r} a_{i}+c$ with $c \in J$, and the claim follows.

With the aid of this lemma, the formula for the ideal $J$ in the above algorithm may be simplified as follows.

Remark 10.9. In the setting of Algorithm 10.7 the potentially expensive compu- 
tation of intersection ideals in the computation of $J$ may be replaced by calculating

$$
\tilde{J}=\sum_{i=1}^{\ell}\left(I_{\mathcal{O O}_{i}^{\prime}}^{\mathrm{dfb}} \cap J_{i}\right)+I\left(\mathbb{B}_{\mathcal{O}}(\mathcal{H})\right) \quad \text { or } \quad \hat{J}=\sum_{i=1}^{\ell}\left(I_{\mathcal{O O}_{i}^{\prime}}^{\mathrm{dfb}} \cdot J_{i}\right)+I\left(\mathbb{B}_{\mathcal{O}}(\mathcal{H})\right)
$$

Then the $K$-rational points of $\mathbb{B}_{\mathcal{O}}(\mathcal{H}) \backslash \mathcal{Z}(J)$ are precisely the points $\Gamma$ in $\mathbb{B}_{\mathcal{O}}(\mathcal{H})$ which represent those affine schemes $\mathbb{X}_{\Gamma}$ that have property $\mathcal{P}$.

\section{The Cayley-Bacharach Locus in $\mathbb{B}_{\mathcal{O}}(\mathcal{H})$}

In this section we use Algorithm 6.3 to find the equations describing the CayleyBacharach locus in the border basis scheme. Since the definition of the CayleyBacharach property involves the regularity index of $R$, we have to fix the Hilbert function $\mathcal{H}$ and work in the subscheme $\mathbb{B}_{\mathcal{O}}(\mathcal{H})$ of the border basis scheme. Furthermore, the characterization of the Cayley-Bacharach property in [14, Thm. 4.5, which underlies Algorithm 6.3 requires us to fix a degree filtered basis of the coordinate ring. Hence we work in the various $\mathcal{O}^{\prime}$-DFB subschemes of $\mathbb{B}_{\mathcal{O}}(\mathcal{H})$, where $\mathcal{O}^{\prime}$ is a degree filtered order ideal for $\mathcal{O}$. The recombination of the individual loci is then achieved using Algorithm 10.7 Thus the result is of the following algorithm is a list of ideals which describes the complement of the Cayley-Bacharach locus in $\mathbb{B}_{\mathcal{O}}(\mathcal{H})$.

\section{Algorithm 11.1. (Computing the Cayley-Bacharach Locus)} Let $\mathcal{O}=\left\{t_{1}, \ldots, t_{\mu}\right\}$ be an order ideal in $\mathbb{T}^{n}$, let $\mathcal{H}=\left(H_{0}, H_{1}, \ldots\right)$ be an $(n, \mu)$-admissible Hilbert function which dominates $\mathrm{HF}_{\mathcal{O}}^{a}$, and let $\varrho=\min \left\{i \geq 0 \mid H_{i}=\mu\right\}$. Consider the following sequence of instructions.

(1) Compute the set $\left\{\mathcal{O}_{1}^{\prime}, \ldots, \mathcal{O}_{\ell}^{\prime}\right\}$ of all order ideals in $\mathbb{T}^{n}$ with affine Hilbert function $\mathcal{H}$.

(2) For $i \in\{1, \ldots, \ell\}$, let $\operatorname{CBLocus}\left(\mathcal{O}_{i}^{\prime}\right)$ be the function obtained by applying Algorithm 6.3 to $\mathcal{O}_{i}^{\prime}$. It yields an ideal $J_{\mathcal{O}_{i}^{\prime}}$ in $K\left[C_{i}^{\prime}\right]$, where $C_{i}^{\prime}$ is the set of indetermintes such that $B_{\mathcal{O}_{i}^{\prime}}=K\left[C_{i}^{\prime}\right] / I\left(\mathbb{B}_{\mathcal{O}_{i}^{\prime}}\right)$.

(3) Apply Algorithm 10.7 using the function $\operatorname{CBLocus}\left(\mathcal{O}_{i}^{\prime}\right)$ in Step (4). Return the resulting list of triples $\left(\mathcal{O}_{i}^{\prime}, I_{\mathcal{O O}_{i}^{\prime}}^{\text {dfb }}, J_{i}\right)$ where $i=1, \ldots, \ell$.

This is an algorithm which computes a list of triples $\left(\mathcal{O}_{i}^{\prime}, I_{\mathcal{O O}_{i}^{\prime}}^{\mathrm{dfb}}, J_{i}\right)$ such that, for the ideal

$$
I_{\mathcal{O}}^{\mathrm{NCB}}=\sum_{i=1}^{\ell}\left(\left(I_{\mathcal{O O}_{i}^{\prime}}^{\mathrm{dfb}}+I\left(\mathbb{B}_{\mathcal{O}}(\overline{\mathcal{H}})\right)\right) \cap\left(J_{i}+I\left(\mathbb{B}_{\mathcal{O}}(\overline{\mathcal{H}})\right)\right)\right)
$$

the set $\mathbb{B}_{\mathcal{O}}(\overline{\mathcal{H}}) \backslash \mathcal{Z}\left(I_{\mathcal{O}}^{\mathrm{NCB}}\right)$ contains precisely the $K$-rational points of $\mathbb{B}_{\mathcal{O}}(\mathcal{H})$ which represent schemes having the Cayley-Bacharach property.

Proof. This follows by combining Algorithm 6.3 and Algorithm 10.7

Note that we may apply the improvement offered by Remark 10.9 to this algorithm. It is also possible to construct a version which avoids the introduction of 
new sets of indeterminates $C_{i}^{\prime}$. Since this version did not yield worthwhile speedups for the actual implementation, we did not include it here. Let us illustrate Algorithm 11.1 by applying it in the setting of Example 10.4

Example 11.2. In $\mathbb{T}^{2}$, we consider the order ideal $\mathcal{O}=\left\{1, x, y, x^{2}, x^{3}\right\}$, and we use the Hilbert function $\mathcal{H}=(1,3,5,5, \ldots)$. As we saw in Example10.4, the scheme $\mathbb{B}_{\mathcal{O}}(\mathcal{H})$ is covered by three open subschemes $\mathbb{B}_{\mathcal{O}}^{\text {dfb }}\left(\mathcal{O}_{i}^{\prime}\right)$ where $i \in\{1,2,3\}$. Let us compute the Cayley-Bacharach locus for each scheme $\mathbb{B}_{\mathcal{O}}^{\mathrm{dfb}}\left(\mathcal{O}_{i}^{\prime}\right)$.

(a) For the order ideal $\mathcal{O}_{1}^{\prime}=\left\{1, x, y, x^{2}, x y\right\}$, the algorithm produces the triple $\left(\mathcal{O}_{1}^{\prime}, I_{\mathcal{O O}_{1}^{\prime}}^{\mathrm{dfb}}, J_{1}\right)$ with $I_{\mathcal{O}}^{\mathrm{dfb}}=\left\langle c_{51}\right\rangle$ and $J_{1}=\langle 1\rangle$.

(b) Similarly, for the order ideal $\mathcal{O}_{2}^{\prime}=\left\{1, x, y, x^{2}, y^{2}\right\}$, we obtain $I_{\mathcal{O O}_{2}^{\prime}}^{\mathrm{dfb}}=\left\langle c_{52}\right\rangle$ and $J_{2}=\langle 1\rangle$.

(c) Thirdly, for $\mathcal{O}_{3}^{\prime}=\left\{1, x, y, x y, y^{2}\right\}$, we get $I_{\mathcal{O O}_{3}^{\prime}}^{\mathrm{dfb}}=\left\langle c_{42} c_{51}-c_{41} c_{52}\right\rangle$ and $J_{3}=\langle 1\rangle$. Using Remark 10.9, the combined result is

$$
\tilde{I}_{\mathcal{O}}^{\mathrm{NCB}}=\sum_{i=1}^{3}\left(I_{\mathcal{O} \mathcal{O}_{i}^{\prime}}^{\mathrm{dfb}} \cap J_{\mathcal{O}_{i}^{\prime}}\right)+I\left(\mathbb{B}_{\mathcal{O}}(\overline{\mathcal{H}})\right)=\left\langle c_{51}, c_{52}\right\rangle+I\left(\mathbb{B}_{\mathcal{O}}(\overline{\mathcal{H}})\right)=I\left(Z_{\mathcal{O}}(\overline{\mathcal{H}})\right)
$$

i.e., an ideal defining the complement of $\mathbb{B}_{\mathcal{O}}(\mathcal{H})$ in $\mathbb{B}_{\mathcal{O}}(\overline{\mathcal{H}})$. This is in agreement with the observation that, for $\mathcal{H}=(1,3,5,5, \ldots)$, every $K$-rational point of $\mathbb{B}_{\mathcal{O}}(\mathcal{H})$ represents a Cayley-Bacharach scheme.

Next we apply Algorithm 11.1 to a case where not every scheme has the CayleyBacharach property.

Example 11.3. Let us consider the order ideal $\mathcal{O}=\left\{1, x, x^{2}, x^{3}\right\}$ in $\mathbb{T}^{2}$ and $\mathcal{H}=(1,3,4,4, \ldots)$. In this case we have three order ideals with affine Hilbert function $\mathcal{H}$, namely $\mathcal{O}_{1}^{\prime}=\left\{1, x, y, x^{2}\right\}, \mathcal{O}_{2}^{\prime}=\{1, x, y, x y\}$, and $\mathcal{O}_{3}^{\prime}=\left\{1, x, y, y^{2}\right\}$. Here Algorithm 11.1 yields the following three triples $\left(\mathcal{O}_{i}^{\prime}, I_{\mathcal{O O}_{i}^{\prime}}^{\mathrm{dfb}}, J_{i}\right)$.

(a) $I_{\mathcal{O O}_{1}^{\prime}}^{\mathrm{dfb}}=\left\langle c_{41}\right\rangle$ and $J_{1}=\langle h\rangle$, where

$$
\begin{aligned}
h= & c_{34}^{2} c_{41}^{4}-2 c_{31} c_{34} c_{41}^{3} c_{44}+c_{31}^{2} c_{41}^{2} c_{44}^{2}-2 c_{31}^{2} c_{34} c_{41}^{2}+2 c_{21} c_{34} c_{41}^{3}+2 c_{31}^{3} c_{41} c_{44} \\
& -2 c_{21} c_{31} c_{41}^{2} c_{44}+c_{31}^{4}-2 c_{21} c_{31}^{2} c_{41}+c_{21}^{2} c_{41}^{2}-c_{21} c_{32} c_{41}^{2}-c_{31} c_{33} c_{41}^{2}-c_{35} c_{41}^{3} \\
& +c_{21} c_{31} c_{41} c_{42}+c_{31}^{2} c_{41} c_{43}+c_{31} c_{41}^{2} c_{45},
\end{aligned}
$$

(b) $I_{\mathcal{O O}_{2}^{\prime}}^{\mathrm{dfb}}=\left\langle c_{34} c_{41}^{2}-c_{31} c_{41} c_{44}-c_{31}^{2}+c_{21} c_{41}\right\rangle$ and $J_{2}=\langle h\rangle$,

(c) $I_{\mathcal{O O}_{3}^{\prime}}^{\mathrm{dfb}}=\left\langle c_{21} c_{32} c_{41}+c_{31} c_{33} c_{41}+c_{35} c_{41}^{2}-c_{21} c_{31} c_{42}-c_{31}^{2} c_{43}-c_{31} c_{41} c_{45}\right\rangle$ and $J_{3}=\langle h\rangle$.

Using Remark 10.9 and computing up to radical, we get

$$
\tilde{I}_{\mathcal{O}}^{\mathrm{NCB}}=\sum_{i=1}^{3}\left(I_{\mathcal{O} \mathcal{O}_{i}^{\prime}}^{\mathrm{dfb}} \cap\langle h\rangle\right)+I\left(\mathbb{B}_{\mathcal{O}}(\overline{\mathcal{H}})\right) \equiv\left\langle c_{31}, c_{41}\right\rangle \cap\langle h\rangle+I\left(\mathbb{B}_{\mathcal{O}}(\overline{\mathcal{H}})\right)
$$

Since $I\left(Z_{\mathcal{O}}(\overline{\mathcal{H}})\right)=\left\langle c_{31}, c_{41}\right\rangle+I\left(\mathbb{B}_{\mathcal{O}}(\overline{\mathcal{H}})\right)$, it follows that the Cayley-Bacharach locus in $\mathbb{B}_{\mathcal{O}}(\mathcal{H})$ is defined by a single polynomial. Using the fact that we can replace $h$ 
by its normal form with respect to $I\left(\mathbb{B}_{\mathcal{O}}(\overline{\mathcal{H}})\right)$, we see that we can also use the polynomial

$$
\tilde{h}=-c_{35} c_{41}^{3}+c_{21} c_{41} c_{42}^{2}+c_{42}^{4}-c_{21} c_{41}^{2} c_{43}+c_{31} c_{41} c_{42} c_{43}-2 c_{41} c_{42}^{2} c_{43}+c_{41}^{2} c_{43}^{2}
$$

to define the Cayley-Bacharach locus in $\mathbb{B}_{\mathcal{O}}(\mathcal{H})$.

If we combine the locally Gorenstein property and the Cayley-Bacharach property, we can compute the corresponding locus by intersecting the loci computed in Algorithm 4.3 and Algorithm 11.1. Another approach, based on [14, Algorithm 5.9, produces a more direct method. Since the resulting algorithm is similar to Algorithm 11.1, we did not include it here.

Another application of Algorithm 11.1 is the following method for calculating the strict Gorenstein locus in $\mathbb{B}_{\mathcal{O}}(\mathcal{H})$. It is based on the fact that a 0 -dimensional affine scheme $\mathbb{X}$ is strictly Gorenstein if and only if its affine Hilbert function is symmetric and it is Cayley-Bacharach (see [14, Thm. 6.8).

\section{Corollary 11.4. (Computing the Strict Gorenstein Locus)}

In the above setting, the following instructions define an algorithm which computes an ideal $I_{\mathcal{O}}^{\mathrm{SG}}$ in $K[C]$ such that the $K$-rational points $\Gamma$ of $\mathbb{B}_{\mathcal{O}}(\mathcal{H}) \backslash \mathcal{Z}\left(I_{\mathcal{O}}^{\mathrm{SG}}\right)$ are precisely the ones which represent 0-dimensional affine schemes $\mathbb{X}_{\Gamma}$ that are strictly Gorenstein.

(1) Check if the affine Hilbert function $\mathcal{H}$ is symmetric. If this is not the case, return the zero ideal and stop.

(2) Using Algorithm 11.1, compute an ideal $I_{\mathcal{O}}^{\mathrm{NCB}}$ in $K[C]$ which defines the nonCayley-Bacharach locus in $\mathbb{B}_{\mathcal{O}}(\mathcal{H})$ and return it.

\section{The Strict Cayley-Bacharach Locus in $\mathbb{B}_{\mathcal{O}}(\mathcal{H})$}

Our next goal is to calculate the strict Cayley-Bacharach locus in $\mathbb{B}_{\mathcal{O}}$. Again we have to fix the Hilbert function first, that is, we have to work in a subscheme $\mathbb{B}_{\mathcal{O}}(\mathcal{H})$ of $\mathbb{B}_{\mathcal{O}}$. Then we use a version of Algorithm 7.10 which is based on a degree filtered border basis. Finally, we recombine the various loci for all degree filtered order ideals. In detail, we have the following algorithm.

\section{Algorithm 12.1. (Computing the Strict Cayley-Bacharach Locus)}

Let $\mathcal{O}=\left\{t_{1}, \ldots, t_{\mu}\right\}$ be an order ideal in $\mathbb{T}^{n}$, let $\mathcal{H}=\left(H_{0}, H_{1}, \ldots\right)$ be an $(n, \mu)$ admissible Hilbert function which dominates $\operatorname{HF}_{\mathcal{O}}^{a}$, let $\varrho=\min \left\{i \geq 0 \mid H_{i}=\mu\right\}$, and let $\Delta=H_{\varrho}-H_{\varrho-1}$. Consider the following sequence of instructions.

(1) Compute the set $\left\{\mathcal{O}_{1}^{\prime}, \ldots, \mathcal{O}_{\ell}^{\prime}\right\}$ of all order ideals in $\mathbb{T}^{n}$ with affine Hilbert function $\mathcal{H}$.

(2) For $i \in\{1, \ldots, \ell\}$, let $\operatorname{SCBLocus}\left(\mathcal{O}_{i}^{\prime}\right)$ be function obtained by applying Algorithm 7.10 to $\mathcal{O}_{i}^{\prime}$. It yields an ideal $J_{\mathcal{O}_{i}^{\prime}}$ in $K\left[C_{i}^{\prime}\right]$, where $C_{i}^{\prime}$ is the set of indetermintes such that $B_{\mathcal{O}_{i}^{\prime}}=K\left[C_{i}^{\prime}\right] / I\left(\mathbb{B}_{\mathcal{O}_{i}^{\prime}}\right)$. 
(3) Apply Algorithm 10.7 using the function SCBLocus $\left(\mathcal{O}_{i}^{\prime}\right)$ in Step (4). Return the resulting list of triples $\left(\mathcal{O}_{i}^{\prime}, I_{\mathcal{O O}^{\prime}}^{\mathrm{dfb}}, J_{i}\right)$, where $i=1, \ldots, \ell$.

This is an algorithm which computes a list of triples $\left(\mathcal{O}_{i}^{\prime}, I_{\mathcal{O O}_{i}^{\prime}}^{\mathrm{dfb}}, J_{i}\right)$ such that, for the ideal

$$
I_{\mathcal{O}}^{\mathrm{NSCB}}=\sum_{i=1}^{\ell}\left(\left(I_{\mathcal{O O}_{i}^{\prime}}^{\mathrm{dfb}}+I\left(\mathbb{B}_{\mathcal{O}}(\overline{\mathcal{H}})\right)\right) \cap\left(J_{i}+I\left(\mathbb{B}_{\mathcal{O}}(\overline{\mathcal{H}})\right)\right)\right)
$$

the set $\mathbb{B}_{\mathcal{O}}(\overline{\mathcal{H}}) \backslash \mathcal{Z}\left(I_{\mathcal{O}}^{\mathrm{NSCB}}\right)$ contains precisely the $K$-rational points of $\mathbb{B}_{\mathcal{O}}(\mathcal{H})$ which represent schemes having the strict Cayley-Bacharach property.

Proof. This follows from Algorithm 7.10 and Algorithm 10.7.

To illustrate this algorithm, we apply it in a concrete case.

Example 12.2. In $\mathbb{T}^{3}$, we consider the order ideal $\mathcal{O}=\left\{1, x, y, z, x^{2}, x^{3}\right\}$, and we let $\mathcal{H}=(1,4,6,6, \ldots)$. Then we have $\varrho=\Delta=2$, the ideal $I\left(\mathbb{B}_{\mathcal{O}}(\overline{\mathcal{H}})\right)$ is generated by 81 polynomials

$$
\begin{aligned}
I\left(\mathbb{B}_{\mathcal{O}}(\overline{\mathcal{H}})=\right. & \left\langle c_{310} c_{61}-c_{28} c_{62}+c_{410} c_{62}-c_{38} c_{64}-c_{48} c_{65}-c_{58} c_{67}+c_{510}, \ldots,\right. \\
& \left.-c_{11} c_{21}-c_{13} c_{31}+c_{11} c_{33}-c_{14} c_{41}+c_{12} c_{43}-c_{16} c_{51}-c_{19} c_{61}+c_{18} c_{63}\right\rangle
\end{aligned}
$$

and the ideal $I\left(Z_{\mathcal{O}}(\overline{\mathcal{H}})\right)$ is generated by 61 polynomials

$$
I\left(Z_{\mathcal{O}}(\overline{\mathcal{H}})\right)=\left\langle c_{65}, c_{64}, \ldots,-c_{11} c_{21}-c_{13} c_{31}+c_{11} c_{33}-c_{14} c_{41}+c_{12} c_{43}-c_{16} c_{66}\right\rangle
$$

Notice that there are 15 order ideals with affine Hilbert function $\mathcal{H}$, namely $\mathcal{O}_{1}^{\prime}=\left\{1, x, y, z, x^{2}, x y\right\}, \mathcal{O}_{2}^{\prime}=\left\{1, x, y, z, x^{2}, y^{2}\right\}, \ldots, \mathcal{O}_{15}^{\prime}=\left\{1, x, y, z, y z, z^{2}\right\}$. Hence Algorithm 12.1 yields 15 triples $\left(\mathcal{O}_{i}^{\prime}, I_{\mathcal{O O}_{i}^{\prime}}^{\text {dfb }}, J_{i}\right)$, where

(1) $I_{\mathcal{O O}_{1}^{\prime}}^{\mathrm{dfb}}=I\left(Z_{\mathcal{O}}(\overline{\mathcal{H}})\right) \cap\left\langle c_{61}\right\rangle$ and

$$
\begin{aligned}
& J_{1}=\left\langle c_{52} c_{62} c_{63}-c_{52} c_{61} c_{64}-c_{51} c_{62} c_{64}+c_{51} c_{61} c_{65}+c_{64}^{2}-c_{63} c_{65},\right. \\
& \\
& c_{52} c_{61} c_{62}-c_{51} c_{62}^{2}+c_{62} c_{64}-c_{61} c_{65}, \\
&-c_{55} c_{61}^{2}+c_{54} c_{61} c_{62}-c_{51} c_{62} c_{64}+c_{51} c_{61} c_{65}+c_{64}^{2}-c_{63} c_{65}, \\
& c_{54} c_{61}^{2}-c_{53} c_{61} c_{62}+c_{51} c_{62} c_{63}-c_{51} c_{61} c_{64}, \\
& c_{52} c_{61}^{2}-c_{51} c_{61} c_{62}+c_{62} c_{63}-c_{61} c_{64}, \\
&-c_{51} c_{52} c_{61} c_{63}+c_{51}^{2} c_{62} c_{63}+c_{54} c_{61} c_{63}-c_{53} c_{62} c_{63}, \\
&\left.c_{51} c_{54} c_{61} c_{62}-c_{51} c_{53} c_{62}^{2}+c_{51} c_{52} c_{61} c_{64}-c_{51}^{2} c_{61} c_{65}-c_{54} c_{61} c_{64}+c_{53} c_{62} c_{64}\right\rangle
\end{aligned}
$$

$$
\begin{aligned}
I_{\mathcal{O O}_{15}^{\prime}}^{\mathrm{dfb}}= & I\left(Z_{\mathcal{O}}(\overline{\mathcal{H}})\right) \cap\left\langle c_{55} c_{64}-c_{54} c_{65}\right\rangle \text { and } \\
J_{15}= & \left\langle c_{55}^{2} c_{62} c_{63}-c_{55}^{2} c_{61} c_{64}-c_{54} c_{55} c_{62} c_{64}+\cdots-c_{51} c_{54} c_{65}^{2}, \quad \ldots,\right. \\
& \left.c_{54}^{3} c_{61} c_{62} c_{64}-c_{53} c_{54}^{2} c_{62}^{2} c_{64}-c_{52} c_{54}^{2} c_{61} c_{64}^{2}+\cdots+c_{53} c_{54} c_{64}^{2} c_{65}\right\rangle
\end{aligned}
$$

where $J_{15}$ has 7 generators of degree 4 and 3 generators of degree 6 . 
Let us compare this result to the ideals defining the Cayley-Bacharach locus in $\mathbb{B}_{\mathcal{O}}(\mathcal{H})$ which we compute using Algorithm 11.1

(1) For $\mathcal{O}_{1}^{\prime}=\left\{1, x, y, z, x^{2}, x y\right\}$ the ideal $\tilde{J}_{1}$ is generated by 58 polynomials

$$
\begin{gathered}
\tilde{J}_{1}=\left\langle c_{52} c_{62} c_{63}-c_{52} c_{61} c_{64}-c_{51} c_{62} c_{64}+c_{51} c_{61} c_{65}+c_{64}^{2}-c_{63} c_{65}, \quad \ldots,\right. \\
\left.c_{31} c_{33} c_{41}^{2} c_{53}^{2} c_{61} c_{62}^{4} c_{63}+c_{33} c_{41}^{3} c_{53}^{2} c_{62}^{5} c_{63}+\cdots-\frac{99}{4} c_{23} c_{53}^{2} c_{63}^{2} c_{65}\right\rangle
\end{gathered}
$$

:

(15) For $\mathcal{O}_{15}^{\prime}=\left\{1, x, y, z, y z, z^{2}\right\}$ the ideal $\tilde{J}_{15}$ is generated by 54 polynomials

$$
\begin{aligned}
\tilde{J}_{15}=\langle & c_{55}^{2} c_{62} c_{63}-c_{55}^{2} c_{61} c_{64}-c_{54} c_{55} c_{62} c_{64}+\cdots-c_{51} c_{54} c_{65}^{2}, \quad \ldots, \\
& c_{24}^{2} c_{34}^{2} c_{52} c_{53}^{2} c_{54} c_{55}^{2} c_{62}^{4} c_{64}^{2}-c_{24}^{2} c_{34}^{2} c_{51} c_{53} c_{54}^{2} c_{55}^{2} c_{62}^{4} c_{64}^{2}+\cdots \\
& \left.+\frac{515}{2} c_{24}^{3} c_{53}^{2} c_{54} c_{64} c_{65}^{5} c_{610}\right\rangle .
\end{aligned}
$$

It is straightforward to check that $J_{i} \subseteq \tilde{J}_{i}$ for $i=1, \ldots, 15$, i.e., that the strict Cayley-Bacharach locus is contained in the Cayley-Bacharach locus of $\mathbb{B}_{\mathcal{O}}(\mathcal{H})$.

To see that these two loci differ, we construct one point in $\mathbb{B}_{\mathcal{O}}(\overline{\mathcal{H}}) \backslash \mathcal{Z}\left(I_{\mathcal{O}}^{\mathrm{NCB}}\right)$ which is not contained in $\mathbb{B}_{\mathcal{O}}(\overline{\mathcal{H}}) \backslash \mathcal{Z}\left(I_{\mathcal{O}}^{\mathrm{NSCB}}\right)$. For this purpose, consider the point $\Gamma=\left(\gamma_{i j}\right)$ such that $\gamma_{32}=\gamma_{61}=\gamma_{67}=1$ and $\gamma_{i j}=0$ otherwise. It represents the scheme $\mathbb{X}$ in $\mathbb{A}_{K}^{3}$ defined by $I_{\mathbb{X}}=\left\langle x y-x^{3}, x z-y, y^{2}, y z, z^{2}, x^{2} y, x^{2} z-\right.$ $\left.x^{3}, x^{4}, x^{3} y, x^{3} z\right\rangle$. It is easy to check that $\Gamma$ is contained in $\mathcal{Z}\left(J_{i}\right)$ for $i=1, \ldots, 15$ and in $\mathcal{Z}\left(I\left(\mathbb{B}_{\mathcal{O}}(\overline{\mathcal{H}})\right)\right)$. Consequently, the point $\Gamma$ is a zero of $I_{\mathcal{O}}^{\text {NSCB }}$, i.e., the scheme $\mathbb{X}$ does not have the strict Cayley-Bacharach property. On the other hand, the point $\Gamma$ is not a zero of $f=c_{32} c_{61}^{2}-c_{31} c_{61} c_{62}+c_{42} c_{61} c_{62}-c_{41} c_{62}^{2}$, and it is straightforward to vertify that $f \in \tilde{J}_{1} \backslash J_{1}$. Hence $\Gamma$ is not contained in $\mathcal{Z}\left(I_{\mathcal{O}}^{\mathrm{NCB}}\right)$, i.e., the scheme $\mathbb{X}$ has the Cayley-Bacharach property.

Remark 12.3. Recall that the strict Gorenstein locus equals the strict CayleyBacharach locus in $\mathbb{B}_{\mathcal{O}}(\mathcal{H})$ if $\Delta=1$, and it is empty otherwise (see [14, Thm. 6.12). Thus Algorithm 12.1 yields an alternative to Corollary 7.13 for computing the strict Gorenstein locus in $\mathbb{B}_{\mathcal{O}}(\mathcal{H})$.

\section{The Strict Complete Intersection Locus in $\mathbb{B}_{\mathcal{O}}(\mathcal{H})$}

The last case that we consider is the locus in $\mathbb{B}_{\mathcal{O}}(\mathcal{H})$ whose $K$-rational points represent 0 -dimensional affine schemes which are strict complete intersections. Clearly, the first necessary condition is that the affine Hilbert function $\mathcal{H}$ has to be symmetric. Then we cover $\mathbb{B}_{\mathcal{O}}(\mathcal{H})$ by $\mathcal{O}^{\prime}$-DFB subschemes and use a suitable version of Algorithm 8.3 in $\mathbb{B}_{\mathcal{O}}^{\text {dfb }}\left(\mathcal{O}^{\prime}\right)$. Lastly, we use Algorthm 10.7 to recombine the results. In this way we arrive at the following algorithm.

\section{Algorithm 13.1. (Computing the Strict Complete Intersection Locus) Let $\mathcal{O}=\left\{t_{1}, \ldots, t_{\mu}\right\}$ be an order ideal in $\mathbb{T}^{n}$, let $\mathcal{H}=\left(H_{0}, H_{1}, \ldots\right)$ be an $(n, \mu)$ - admissible Hilbert function which dominates $\mathrm{HF}_{\mathcal{O}}^{a}$, let $\varrho=\min \left\{i \geq 0 \mid H_{i}=\mu\right\}$, and let $\Delta=H_{\varrho}-H_{\varrho-1}$. Consider the following sequence of instructions.}


(1) Check if $\mathcal{H}$ is symmetric. If this is not the case, return the triple $\left(\mathcal{O}, I\left(B_{\mathcal{O}}(\overline{\mathcal{H}})\right),\langle 0\rangle\right)$ and stop.

(2) Compute the set $\left\{\mathcal{O}_{1}^{\prime}, \ldots, \mathcal{O}_{\ell}^{\prime}\right\}$ of all order ideals in $\mathbb{T}^{n}$ with affine Hilbert function $\mathcal{H}$.

(3) For $i \in\{1, \ldots, \ell\}$, let $\operatorname{SCILocus}\left(\mathcal{O}_{i}^{\prime}\right)$ be function obtained by applying Algorithm 8.3 to $\mathcal{O}_{i}^{\prime}$. It yields an ideal $J_{\mathcal{O}_{i}^{\prime}}$ in $K\left[C_{i}^{\prime}\right]$, where $C_{i}^{\prime}$ is the set of indetermintes such that $B_{\mathcal{O}_{i}^{\prime}}=K\left[C_{i}^{\prime}\right] / I\left(\mathbb{B}_{\mathcal{O}_{i}^{\prime}}\right)$.

(4) Apply Algorithm 10.7 using the function $\operatorname{SCILocus}\left(\mathcal{O}_{i}^{\prime}\right)$ in Step (4). Return the resulting list of triples $\left(\mathcal{O}_{i}^{\prime}, I_{\mathcal{O O}_{i}^{\prime}}^{\mathrm{dfb}}, J_{i}\right)$, where $i=1, \ldots, \ell$.

This is an algorithm which computes a list of triples $\left(\mathcal{O}_{i}^{\prime}, I_{\mathcal{O O}_{i}^{\prime}}^{\mathrm{dfb}}, J_{i}\right)$ such that, for the ideal

$$
I_{\mathcal{O}}^{\mathrm{NSCI}}=\sum_{i=1}^{\ell}\left(\left(I_{\mathcal{O O}_{i}^{\prime}}^{\mathrm{dfb}}+I\left(\mathbb{B}_{\mathcal{O}}(\overline{\mathcal{H}})\right)\right) \cap\left(J_{i}+I\left(\mathbb{B}_{\mathcal{O}}(\overline{\mathcal{H}})\right)\right)\right)
$$

the set $\mathbb{B}_{\mathcal{O}}(\overline{\mathcal{H}}) \backslash \mathcal{Z}\left(I_{\mathcal{O}}^{\mathrm{NSCI}}\right)$ contains precisely the $K$-rational points of $\mathbb{B}_{\mathcal{O}}(\mathcal{H})$ which represent schemes having the strict complete intersection property.

Proof. Since the affine Hilbert function $\mathcal{H}$ of a 0 -dimensional strict complete intersection is symmetric, Step (1) returns the correct result when $\mathcal{H}$ is not symmetric. The remaining claims follow from Algorithm 8.3 and Algorithm 10.7.

Let us use this algorithm to compute the strict complete intersection locus in a simple example.

Example 13.2. Consider the order ideal $\mathcal{O}=\left\{1, x, y, x^{2}, x^{3}, x^{4}\right\}$ in $\mathbb{T}^{2}$ and the Hilbert function $\mathcal{H}=(1,3,5,6,6, \ldots)$ which dominates $\operatorname{HF}_{\mathcal{O}}^{a}=(1,3,4,5,6,6, \ldots)$. We apply Algorithm 13.1 and note the main results.

(1) The difference function of $\mathcal{H}$ is $(1,2,2,1,0, \ldots)$ and thus symmetric.

(2) We find six order ideals, namely $\mathcal{O}_{1}^{\prime}=\left\{1, x, y, x^{2}, x y, x^{3}\right\}, \quad \mathcal{O}_{2}^{\prime}=$ $\left\{1, x, y, x^{2}, x y, x^{2} y\right\}, \mathcal{O}_{3}^{\prime}=\left\{1, x, y, x^{2}, y^{2}, x^{3}\right\}, \mathcal{O}_{4}^{\prime}=\left\{1, x, y, x^{2}, y^{2}, y^{3}\right\}, \mathcal{O}_{5}^{\prime}=$ $\left\{1, x, y, x y, y^{2}, x y^{2}\right\}$, and $\mathcal{O}_{6}^{\prime}=\left\{1, x, y, x y, y^{2}, y^{3}\right\}$.

(3) Using $\operatorname{SCILocus}\left(\mathcal{O}_{i}^{\prime}\right)$ for $i=1, \ldots, 6$, we get the following results:

$I_{\mathcal{O O}_{1}^{\prime}}^{\mathrm{dfb}}=\left\langle c_{61}\right\rangle$ and $J_{1}=\left\langle c_{55}^{2} c_{61}^{4}-2 c_{51} c_{55} c_{61}^{3} c_{65}+c_{51}^{2} c_{61}^{2} c_{65}^{2}+\cdots-c_{42} c_{61}^{2}\right.$, $\left.c_{55}^{2} c_{61}^{3} c_{62}-2 c_{51} c_{55} c_{61}^{2} c_{62} c_{65}+c_{51}^{2} c_{61} c_{62} c_{65}^{2}+\cdots-c_{42} c_{61} c_{62}\right\rangle$

$I_{\mathcal{O O}_{2}^{\prime}}^{\mathrm{dfb}}=\left\langle c_{55} c_{61}^{2}-c_{51} c_{61} c_{65}-c_{51}^{2}+c_{41} c_{61}\right\rangle$ and $J_{2}=\left\langle c_{55}^{2} c_{61}^{4}-2 c_{51} c_{55} c_{61}^{3} c_{65}\right.$ $\left.+c_{51}^{2} c_{61}^{2} c_{65}^{2}+\cdots-c_{42} c_{61}^{2}, c_{45} c_{52} c_{55} c_{61}^{4}-c_{45} c_{51} c_{55} c_{61}^{3} c_{62}+\cdots-c_{41} c_{42} c_{61} c_{62}\right\rangle$

$$
\begin{aligned}
& I_{\mathcal{O O}_{6}^{\prime}}^{\mathrm{dfb}}=\left\langle c_{42} c_{43} c_{52} c_{61}+c_{44} c_{52}^{2} c_{61}-\cdots-c_{41} c_{52} c_{62} c_{66}\right\rangle \text { and } J_{6}= \\
& \left\langle c_{45}^{2} c_{52}^{2} c_{61}^{2} c_{62}^{2}-2 c_{42} c_{45} c_{52} c_{55} c_{61}^{2} c_{62}^{2}+\cdots-c_{22} c_{51} c_{52} c_{62}^{2} c_{66}, c_{45}^{2} c_{52}^{2} c_{61}^{3} c_{62}-\right. \\
& \left.\cdots-c_{21} c_{51} c_{52} c_{62}^{2} c_{66}\right\rangle
\end{aligned}
$$


Thus we obtain 6 triples $\left(\mathcal{O}_{i}^{\prime}, I_{\mathcal{O O}_{i}^{\prime}}^{\mathrm{dfb}}, J_{i}\right)$, and using Remark 10.9, we get that

$$
I_{\mathcal{O}}^{\mathrm{NSCI}}=\sum_{i=1}^{6} I_{\mathcal{O O}_{i}^{\prime}}^{\mathrm{dfb}} \cdot J_{i}+I\left(\mathbb{B}_{\mathcal{O}}(\overline{\mathcal{H}})\right)
$$

defines the complement of the strict complete intersection locus in $\mathbb{B}_{\mathcal{O}}(\overline{\mathcal{H}})$. Here $\sum_{i=1}^{6} I_{\mathcal{O O}_{i}^{\prime}}^{\mathrm{dfb}} \cdot J_{i}$ is generated by 14 polynomials, and the ideal $I\left(\mathbb{B}_{\mathcal{O}}(\overline{\mathcal{H}})\right)$ is generated by 30 polynomials

$$
\begin{aligned}
& I\left(\mathbb{B}_{\mathcal{O}}(\overline{\mathcal{H}})\right)=\left\langle c_{52} c_{61}-c_{51} c_{62}, c_{34} c_{61}+c_{64} c_{65}+c_{54}-c_{66}, \quad \ldots,\right. \\
&\left.c_{11} c_{21}+c_{12} c_{31}-c_{11} c_{32}+c_{13} c_{41}+c_{14} c_{51}+c_{16} c_{61}-c_{15} c_{62}\right\rangle
\end{aligned}
$$

This example also shows that even if an order ideal $\mathcal{O}$ does not have a symmetric Hilbert function, there may exist a strict complete intersection scheme $\mathbb{X}_{\Gamma}$ represented by a $K$-rational point $\Gamma$ in $\mathbb{B}_{\mathcal{O}}$. Clearly, this point $\Gamma$ is then not contained in $\mathbb{B}_{\mathcal{O}}^{\text {df }}$.

In more detail, notice that $\mathrm{HF}_{\mathcal{O}}^{a}$ is not symmetric, but the affine Hilbert function $\mathcal{H}$, which dominates $\mathrm{HF}_{\mathcal{O}}^{a}$, is symmetric. Let us consider the 0-dimensional subscheme $\mathbb{X}$ of $\mathbb{A}_{K}^{2}$ defined by $I_{\mathbb{X}}=\left\langle x^{3}-x y, y^{2}\right\rangle$. Then the degree forms $x^{3}, y^{2}$ are a homogeneous regular sequence, and hence $\mathbb{X}$ is a strict complete intersection scheme. For the order ideal $\mathcal{O}=\left\{1, x, y, x^{2}, y^{2}, x^{3}, x^{4}\right\}$, the ideal $I_{\mathbb{X}}$ has an $\mathcal{O}$-border basis, namely

$$
G=\left\{y^{2}, x^{3}-x y, x y^{2}, x^{3} y-x^{2} y, x^{2} y^{2}\right\}
$$

Thus the point $\Gamma$ of $\mathbb{B}_{\mathcal{O}}$ which represents $\mathbb{X}$ is contained in the strict complete intersection locus of $\mathbb{B}_{\mathcal{O}}(\mathcal{H})$. However, the ideal $\operatorname{DF}\left(I_{\mathbb{X}}\right)=\left\langle x^{3}, y^{2}\right\rangle$ has no $\mathcal{O}$-border basis, since $x^{3} \in \mathcal{O}$. Thus the point $\Gamma$ is not contained in $\mathbb{B}_{\mathcal{O}}^{\text {df }}$.

\section{Acknowledgements}

The first and second authors were partially supported by the Vietnam National Foundation for Science and Technology Development (NAFOSTED) grant number 101.04-2019.07. The third author would like to thank the University of Passau for its hospitality and support during part of the preparation of this paper. The authors are indebted to the referee for several insightful and useful remarks.

\section{References}

[1] J. Abbott, A.M. Bigatti, and L. Robbiano, CoCoA: a system for doing Computations in Commutative Algebra, available at http://cocoa.dima.unige.it

[2] C. Bertone, F. Cioffi, and M. Roggero, Smoothable Gorenstein points via marked Schemes and double-generic initial ideals, Exp. Math. (2019), DOI: 10.1080/10586458.2019.1592034

[3] G. Casnati, J. Jelisiejew, and R. Notari, Irreducibility of the Gorenstein loci of Hilbert schemes via ray families, Algebra \& Number Theory 9 (2015), 1525-1570. 
[4] G. Casnati, and R. Notari, On the Gorenstein locus of some punctual Hilbert schemes. J. Pure Appl. Algebra 213 (2009), 2055-2074.

[5] G. Casnati, and R. Notari, On the irreducibility and the singularities of the Gorenstein locus of the punctual Hilbert scheme of degree 10, J. Pure Appl. Algebra 215 (2011), 1243-1254.

[6] D. Eisenbud, Commutative Algebra with a View Toward Algebraic Geometry, Graduate Texts in Math. 150, Springer-Verlag, New York 1995.

[7] V. Drinfeld, On algebraic spaces with an action of $G_{m}$, preprint 2013, available at arXiv:math 1308.2604 [math.AG].

[8] J. Jelisiejew and $\mathrm{L}$. Sienkiewicz. Białynicki-Birula decomposition for reductive groups, J. Math. Pures Appl. 131 (2019), 290-325.

[9] A. Grothendieck, Techniques de construction et théorèmes d'existence en géométrie algébrique IV: les schémas de Hilbert, Asterisque 6 (1960-1961), 249-276.

[10] M. Haiman, t,q-Catalan numbers and the Hilbert scheme, Discrete Math. 193 (1998), 201-224.

[11] M. Huibregtse, An elementary construction of the multigraded Hilbert scheme of points, Pacific J. Math. 223 (2006), 269-315.

[12] M. Huibregtse, The cotangent space at a monomial ideal of the Hilbert scheme of points of an affine space, preprint 2005, available at arxiv:math/0506.575 [math. AG] .

[13] A. Iarrobiano and V. Kanev, Power sums, Gorenstein algebras and determinantal loci, Lect. Notes Math. 1721, Springer-Verlag, Berlin, 1999.

[14] M. Kreuzer, L.N. Long, and L. Robbiano, On the Cayley-Bacharach property, Comm. Algebra 47 (2019), 328-354.

[15] M. Kreuzer, L.N. Long, and L. Robbiano, Algorithms for checking zero-dimensional complete intersections, available at arxiv:math/1903.09563 [math.AG], (to appear in J. Commut. Algebra)

[16] M. Kreuzer, L.N. Long, and L. Robbiano, Computing subschemes of the border basis scheme (2017-2019), https://www.symbcomp.fim.uni-passau.de/en/projects/

[17] M. Kreuzer and L. Robbiano, Computational Commutative Algebra 1, Springer, Heidelberg, 2000.

[18] M. Kreuzer and L. Robbiano, Computational Commutative Algebra 2, Springer, Heidelberg, 2005.

[19] M. Kreuzer and L. Robbiano, Computational Linear and Commutative Algebra, Springer, Heidelberg, 2016.

[20] M. Kreuzer and L. Robbiano, Deformations of border bases, Collect. Math. 59 (2008), 275-297.

[21] M. Kreuzer and L. Robbiano, The geometry of border bases, J. Pure Appl. Algebra 215 (2011), 2005-2018.

[22] L. Robbiano, On border basis and Gröbner basis schemes, Collect. Math. 60 (2009), 11-25.

[23] B. Sipal, Border basis schemes, dissertation, University of Passau, Passau 2017. 